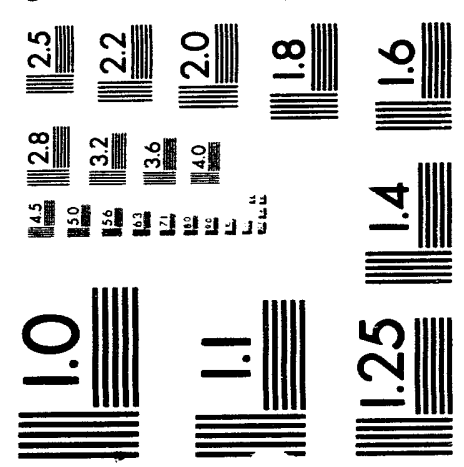



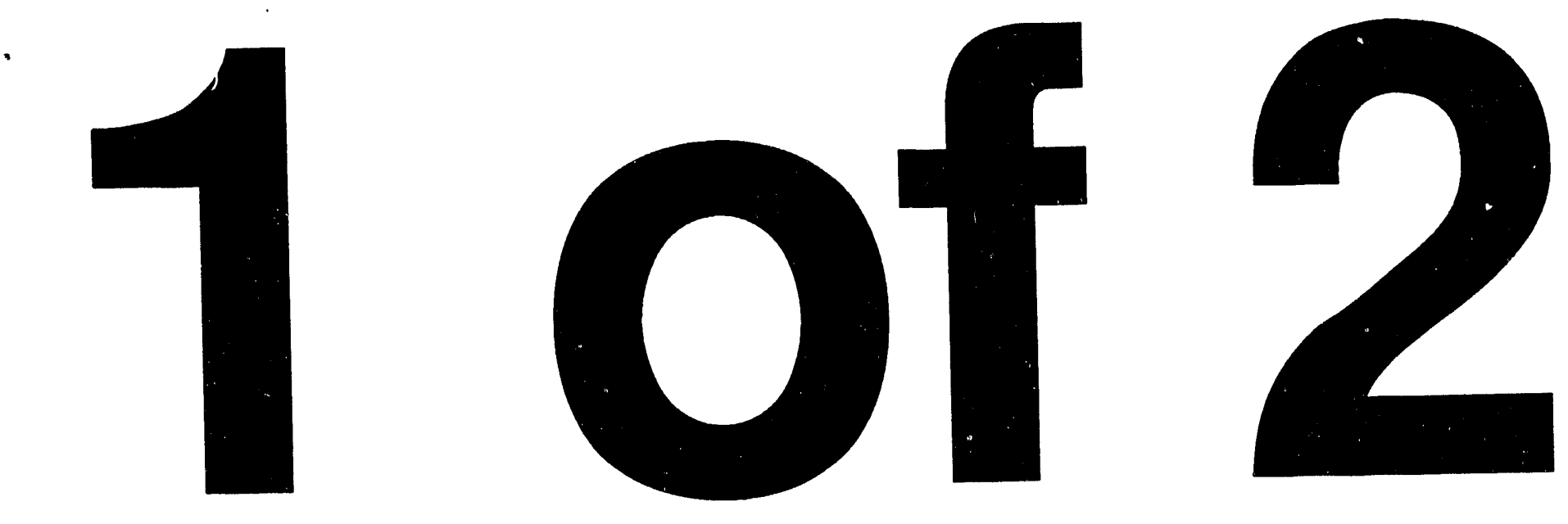


\title{
Chemically Modified Polymeric Resins for Seperation of Cations, Organic Acids, and small Polar Moleculea by High Performance Liquid Chromatography
}

\author{
by John Morris
}

thesis submitted to Iowa State University

Ames Laboratory, U. S. DOE

Iowa State University

Ames, Iowa 50011

Date Transmitted: July 1993

Prepared for the U. S. Department of Energy

Under contract no. W-7405-eng-82.

\section{DISCLAIMER}

This report was prepared as an account of work sponsored by an agency of the United States Government. Neither the United States Government nor any agency thereof, nor any of their employees, makes any warranty, express or implied, or assumes any legal liability or responsibility for the accuracy, completeness, or usefuiness of any information, apparatus, product, or process disclosed, or represents that its use would not infringe privately owned rights. Reference herein to any specific commercial product, process, or service by trade name, trademark, manufacturer, or otherwise does not necessarily constitute or imply its endorsement, recommendation, or favoring by the United States Government or any agency thereof. The views and opinions of authors expressed herein do not necessarily state or reflect those of the United States Government or any agency thereof.

\section{MASTER}


Chemically modified polymeric resins

for separation of cations, organic acids,

and small polar molecules

by high performance liquid chromatography

\section{John Brennan Morris}

\section{Under the supervision of James S. Fritz From the Department of Chemistry Iowa State University}

Simple organic reactions are used to chemically modify macroporous polystyrene -divinylbenzene resins. Cation separations are studied on weak-acid resins that are easily synthesized and carry the exchange group on the free aromatic portion of the repeating styrene unit of the resin or on a short spacer arm from the ring. The synthesis and general properties of two carboxylic acid resins are described. A higher exchange capacity is required for effective ion chromatographic separations than that needed for sulfonated resins. A number of excellent separations are reported using eluents containing ethylenediammonium cations plus a weak complexing agent. The effect of $\mathrm{pH}$ on retention times of metal ions is reported.

Separation of carboxylic acids and other polar compounds by ion-exclusion chromatography is usually performed on a gel-type resin column of high cation exchange capacity (either sulfonated or carboxylated). It is now shown that excellent separations can be obtained on smaller columns packed with lightly sulfonated macroporous resins. The mechanism appears to be a partitioning between the 
predominantly aqueous eluent and the resin matirx. Good separations were also obtained on a low-capacity macroporous resin containing a carboxylic acid functional group. Aqueous-acetonitrile eluent gradients can be used to obtain fast separation of at least 11 different carboxylic acids in a single run.

Small straight-chain alcohols added to an aqueous mobile phase were found to have a dramatic effect on the chromatographic behavior of alkane carboxylic acids. The alcohols were believed to coat the resin surface and establish a dynamic equilibrium between the mobile and stationary phases. This altered the hydrophilicity of the resin surface permitting a fine-tuning of the stationary phase capacity behavior. The eluent systems have a very low background conductance, thus allowing very sensitive conductometric detection. The presence of carbonic acid (from atmospheric carbon dioxide) in the eluent was determined to play a role in the retention mechanism of the weak-acid species. The use of larger straight-chain alcohols as modifiers in a water/acetonitrile mobile phase was investigated for the separation of neutral organic species. Finally, a 12-carbon alcohol was semi-permanently coated on the PS-DVB resin. This treated column was used for separation of alkane carboxylic acids using a purely aqueous eluent and conductometric detection. 
ii

This work is dedicated to my late father, Thomas.

His patience, encouragement, and love

made an everlasting impression on me. 


\section{TABLE OF CONTENTS}

GENERAL INTRODUCTION $\ldots \ldots \ldots \ldots \ldots \ldots \ldots \ldots \ldots \ldots \ldots$

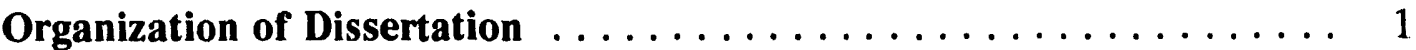

High Performance Liquid Chromatography $\ldots \ldots \ldots \ldots \ldots \ldots$

Polymeric Resins .......................... 3

Ion-Exchange Chromatography of Metal Cations . . . . . . . . . . 7

Ion-Exclusion Chromatography of Organic Acids . . . . . . . . . . 11

Effect of Eluent Modifiers on HPLC Separations . . . . . . . . . . . . 17

PAPER I. ION CHROMATOGRAPHY OF METAL CATIONS ON

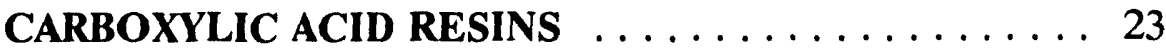

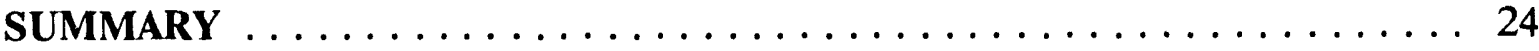

INTRODUCTION $\ldots \ldots \ldots \ldots \ldots \ldots \ldots \ldots \ldots \ldots \ldots \ldots \ldots \ldots \ldots \ldots \ldots \ldots \ldots, 25$

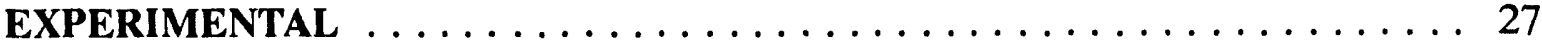

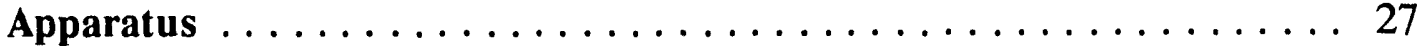

Reagents and chemicals ...................... 27

Synthesis procedures and capacity determination ............ 28

Column packing procedure . . . . . . . . . . . . . . . . . . . 29

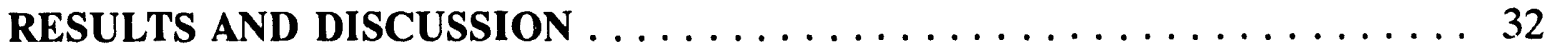

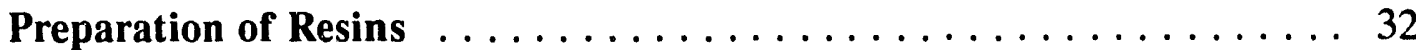

Optimization of Separation Conditions and Effect of pH ....... 32

Citrate systems ........................ 40

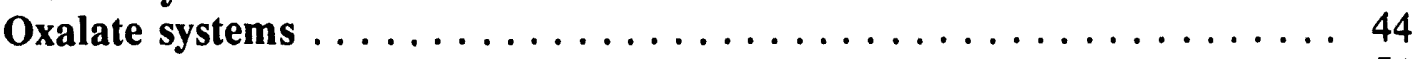

Pyridine-2,6-dicarboxylic acid (PDA) systems . . . . . . . . . 51

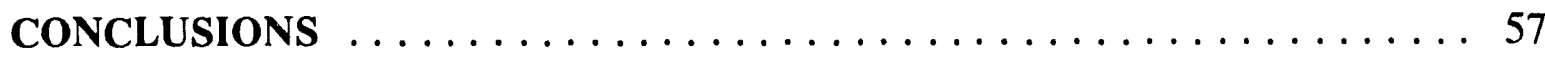

REFERENCES $\ldots \ldots \ldots \ldots \ldots \ldots \ldots \ldots \ldots \ldots \ldots \ldots \ldots \ldots$

PAPER II. SEPARATION OF HYDROPHILIC ORGANIC ACIDS AND SMALL POLAR COMPOUNDS ON

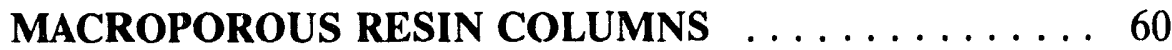

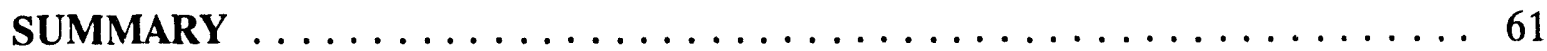

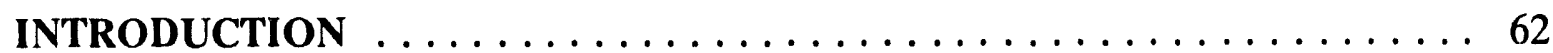

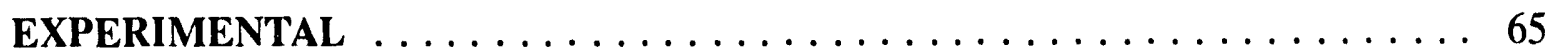




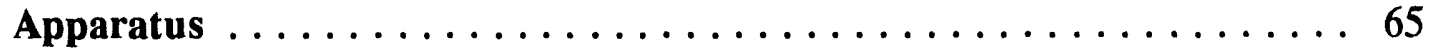

Reagents and chemicals . .................... 66

Synthesis procedures and capacity determination $\ldots \ldots \ldots \ldots \ldots 66$

RESULTS AND DISCUSSION $\ldots \ldots \ldots \ldots \ldots \ldots \ldots \ldots \ldots \ldots \ldots \ldots$

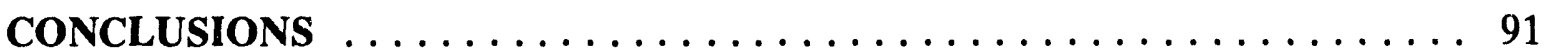

REFERENCES $\ldots \ldots \ldots \ldots \ldots \ldots \ldots \ldots \ldots \ldots \ldots \ldots \ldots \ldots \ldots$

PAPER III. USE OF ELUENT MODIFIERS FOR THE LIQUID CHROMATOGRAPHIC SEPARATION OF CARBOXYLIC ACIDS USING CONDUCTIVITY DETECTION ...... 93

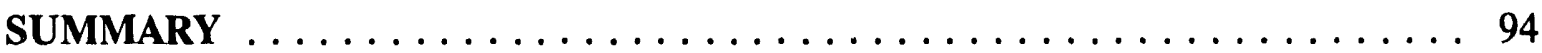

INTRODUCTION $\ldots \ldots \ldots \ldots \ldots \ldots \ldots \ldots \ldots \ldots \ldots \ldots \ldots \ldots$

EXPERIMENTAL $\ldots \ldots \ldots \ldots \ldots \ldots \ldots \ldots \ldots \ldots \ldots \ldots \ldots, 98$

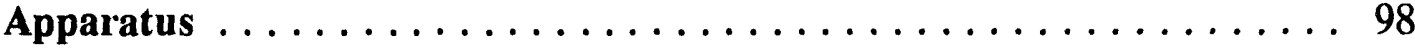

Reagents and chemicals $\ldots \ldots \ldots \ldots \ldots \ldots \ldots \ldots \ldots \ldots, 98$

RESULTS AND DISCUSSION $\ldots \ldots \ldots \ldots \ldots \ldots \ldots \ldots \ldots \ldots \ldots$

Dynamic Coatings with Alcohol Modifiers $\ldots \ldots \ldots \ldots \ldots \ldots \ldots .99$

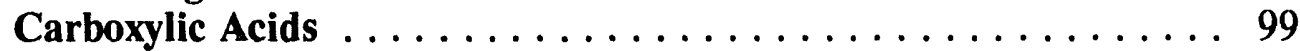

Mechanism of Alcohol Modifiers . . . . . . . . . . . . . . . 112

Neutral Aromatic Compounds ..................... 114

Semi-Permanent Coatings with Alcohol Modifiers $\ldots \ldots \ldots \ldots \ldots 130$

Effect of Carbonic Acid on Retention Mechanism . . . . . . . . . . . 133

Dynamic Alcohol Coating . . . . . . . . . . . . . . . 133

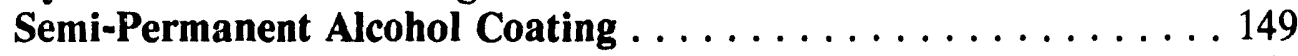

Quantitative Chromatographic Determinations $\ldots \ldots \ldots \ldots \ldots \ldots 153$

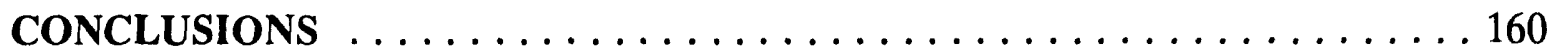

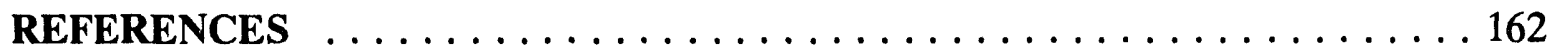

GENERAL CONCLUSIONS $\ldots \ldots \ldots \ldots \ldots \ldots \ldots \ldots \ldots \ldots \ldots \ldots$

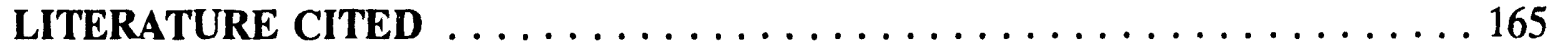

ACKNOWLEDGEMENTS $\ldots \ldots \ldots \ldots \ldots \ldots \ldots \ldots \ldots \ldots \ldots \ldots \ldots$ 


\section{GENERAL INTRODUCTION}

\section{Organization of Dissertation}

This dissertation starts with a general introduction containing a literature review. This is followed by two research papers which have been published in analytical chemistry journals and a third which will soon be submitted for publication. Permission from the publisher extending reproduction and distribution rights is acknowledged. Each paper is preceded by a summary and followed by conclusions. The three papers are followed by a general conclusions section. References cited in the general introduction are compiled in the literature-cited section following general conclusions. Each paper appears in its entirety including references and figures. Figures are contained in the text of the paper at the appropriate location. References cited within each paper are listed after the conclusions of each paper.

\section{High Performance Liquid Chromatography}

High performance liquid chromatography (HPLC) has grown tremendously since its inception in the early 1970 's. Although gas chromatographs were available in the 1960 's, there was an obvious need for an instrumental technique that could separate water soluble, thermally labile, non-volatile compounds with speed, precision, and high resolution. The development of HPLC was stimulated by the discovery of DNA. A reliable technique was needed to separate and quantify small amounts of nucleotides and nucleosides from the hydrolysates of DNA and RNA (1). Since its early beginnings, HPLC has expanded to include more specific modes of operation such as adsorption, ion-exchange, normal-phase, reversed-phase, size-exclusion, ion-exclusion, 
affinity, immunoaffinity, and chiral chromatography. Undoubtedly, the technique of HPLC is now routinely used in most analytical, biochemical, pharmaceutical, medical, and many other industrial laboratories. Although HPLC has become a well established technique, advances are still being made in the fundamental understanding of various separation mechanisms as well as improvements in equipment capabilities.

The most widely used detector in the field is the UV-vis detector which is routinely used for determination of compounds containing a UV chromophore. Excellent progress has been made in the area of specific detectors. Laser induced fluorescence detectors (2), indirect fluorescence detection (3), radioactivity detectors (4), and electrochemical detectors (5-7) have all shown marked improvement for detection of specific classes of compounds.

The power of HPLC as an analytical technique has been increased by its coupling to other analytical methods. For example, HPLC coupled to mass spectrometry allows the simultaneous separation and determination of molecular structures of sample components (8). Microcolumn HPLC also facilitates coupling to other detectors such as fourier transform infrared (FTIR) (9) or with other techniques such as capillary electrophoresis (CE) (10) or gas chromatography (GC) (11).

Stated most simply, a mixture of analyte species is separated by HPLC through some type of partitioning process of the analyte between the mobile phase and the stationary phase. An overwhelming number of stationary phases or packing materials have been developed. Some are highly specialized for specific applications and some are general-purpose. There are specialty packings for affinity $(12,13)$ and 
immunoaffinity modes (14), chiral separations (15), and macromolecules (16). Mixedmode (17) and mixed-bed packings (18) have also been investigated. Currently, the majority of HPLC separations are performed on columns packed with a silica-based support. Supports besides the commonly used silica include other oxides (19), carbon (20), hydroxyapatite beads (21), agarose (22), zirconia (23), and a variety of polymeric resins (24). Work described in this dissertation focuses on the special use of the most widely used polymeric resin. Polystyrene-divinylbenzene resins are chemically modified and used for separation of both ionic and neutral species by various modes of HPLC.

\section{Polymeric Resins}

Silica-based columns are generally more efficient than columns containing polymeric resins such as polystyrene-divinylbenzene (25). However, silica-based resins have some drawbacks that make polymeric resins more attractive for certain applications. The instability of silica in alkaline or highly acidic media is the most serious problem. Using low-pH mobile phases, aggressive solvents, and high temperatures can improve some separations but often creates problems with column stability. Problems with stability are associated with loss of organosilane from the surface of the silica support caused by hydrolysis of the siloxane bond (26). In addition, silica-based packings may also possess residual silanol groups. Separation of ionic or ionizable compounds using a mobile phase with $\mathrm{pH}$ 4-7 can produce tailing and broad peaks (27). However, an improved silica-based packing material with a sterically protected C18 packing for reversed-phase HPLC has recently been reported (28). Finally, after gradient elution, silica-based columns may require extensive treatment to 
re-establish equilibrium for subsequent runs.

Modern polymeric resins, on the other hand, are not affected by the $\mathrm{pH}$ of the mobile phase. Many polymers are stable throughout the $\mathrm{pH}$ range of $0-14$. Therefore, for applications in which column stability is important, and for most applications involving ions, polymeric resins are the appropriate choice.

A large number of polymeric resins with various monomers and crosslinking units have been synthesized for use in HPLC (29). The most popular polymeric resin is polystyrene-divinylbenzene (PS-DVB) synthesized by co-polymerizing a styrene monomer with a divinylbenzene crosslinker. The basic structure of PS-DVB is illustrated in Figure 1. The effect of the mode of synthesis on the characteristics of this cross-linked polymer was examined (30).

Two basic types of PS-DVB are used in HPLC. Microporous PS-DVB is a geltype resin in which the amount of crosslinking agent determines the porosity. Lower crosslinking yields larger porosity and results in a softer product. When dry, these microporous resins have little or no porosity. Upon wetting, the polymer chains become solvated to give what is called a "swelling porosity". Macroporous PS-DVB resins, on the other hand, are synthesized in the presence of a porogen to give a highly crosslinked, rigid, copolymer. The porogen is an inert compound that is soluble in the monomer mixture, remains chemically unbound to the polymer network, and is easily removed from the polymer following polymerization. The amount of crosslinking in PS-DVB can reach 55\% at which the copolymer shows practically no swelling and possesses a permanent porosity (30). 
5

Figure 1. Structure of polystrene-divinylbenzene (PS-DVB) resin 


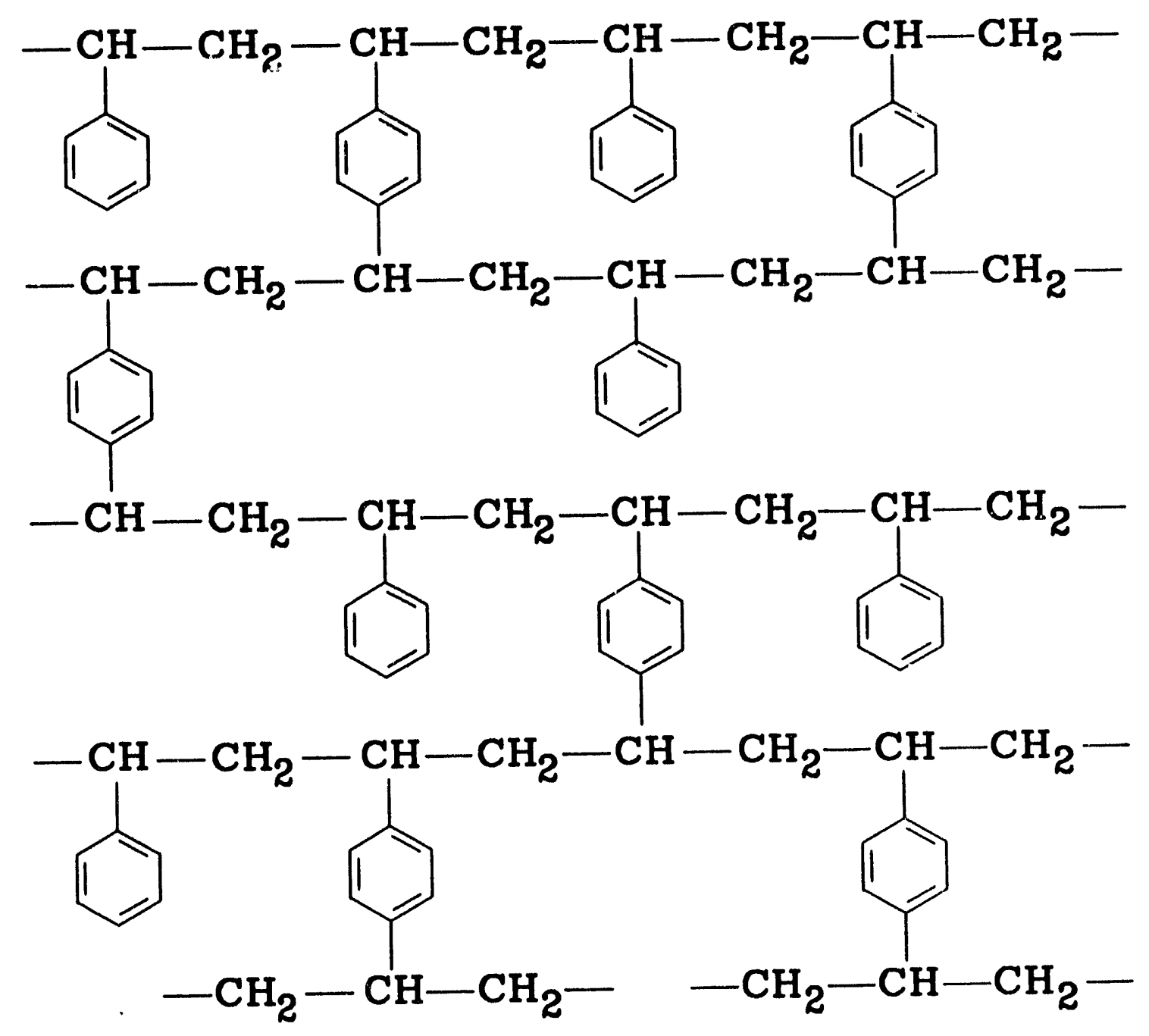


The pore structure of PS-DVB and its influence on chromatographic behavior was investigated (31). The partitioning (adsorption) process of solutes was described as involving the whole bulk mass of the polymer. In other words, solutes can partition with both the outer surface and the polymer matrix encountered upon penetration into the pores of the polymer. The porosity in microporous PS-DVB resins is not constant but can change with the nature of the eluent and sample. It was for this reason that macroporous PS-DVB resins were chosen for work described in this dissertation. The highly crosslinked macroporous PS-DVB resins are very rigid making them capable of tolerating large changes in solvent polarity. This becomes especially important for applications involving gradient elution with an organic solvent.

In all of the papers of this dissertation, the macroporous PS-DVB resins were chemically modified to introduce either carboxylic acid or sulfonic acid functional groups. Underivatized PS-DVB resins tend to be very hydrophobic in nature. The presence of these hydrophilic functional groups makes the PS-DVB resin more wettable by the primarily aqueous eluents, thus providing more intimate contact and possible selective intermolecular interaction with solute species in the mobile phase.

\section{Ion-Exchange Chromatography of Metal Cations}

Modern ion chromatography has transformed into a routine technology since it was first described by Small, Stevens, and Bauman in 1975 (32). The initial ion chromatography system was a dual-column configuration with a suppressor column following the analytical column. This allowed the use of conductivity detection by suppressing the background conductance of the eluent. 
J.S. Fritz and co-workers at Iowa State University found that ion chromatographic analyses of anions and cations could be performed on a single column of sufficiently low exchange capacity and a very dilute eluent (33-35). It is recognized that the ion-exchange process occurs with the hydrogen ions of the resin's acid functional groups that are far inside the pores of the resin as well as with those on the outermost surface of the resin. However, during the derivatization process for lowcapacity resins, the most accessible aromatic rings on the outermost surface of the resin react first. Thus, the majority of ion-exchange sites are located on the outer surface of the resin bead (36). This provides for intimate contact between the resin and the mobile phase and rapid mass transfer for exchange of analyte ions.

The single column ion chromatography (SCIC) configuration probably accounts for most types of IC analyses performed today. However, suppressor technology has steadily improved. Most recently an electrodialytically operated membrane suppressor was developed by Strong and Dasgupta $(37,38)$. This unit is now commercially available. Once again, a high exchange capacity column can be used since the membrane suppressor can adequately handle even gradient elution.

Jones et al. (39) devised an alternative method for gradient elution in the singlecolumn mode using a gradient between two eluents of equal conductivity. The strong eluent contained a greater eluent ion oncentration but had a lower mobility counterion $\left(\mathrm{Li}^{+}\right)$as the cation. Therefore, the conductivity was the same as that of the weaker eluent in the higher mobility $\mathrm{Cs}^{+}$form. 
A review by Fritz (40) described the basic principles of ion chromatography and included a discussion on column packings and methods of detection. A more recent review by Dasgupta (41) discussed ion chromatography coupled to inductively coupled plasma-optical emission spectrometry (ICP-OES) and inductively coupled plasma-mass spectrometry (ICP-MS). Recent advances in interfacing IC with MS and some unique post-suppressor manipulations were also reviewed.

A general system for separation of metal cations includes a low-capacity ionexchange column with either a conductivity or a UV-vis detector. Monovalent cations are typically separated using an eluent of a dilute mineral acid in the $1-10 \mathrm{mM}$ range (42) and amines such as ethylenediammonium salts at $\mathrm{pH} \mathrm{4-6}$ are used for divalent cations. In both cases, the eluent cations have a higher equivalent conductance than the sample cations. This results in negative peaks (decreased conductance) as the sample cations pass through the conductivity detector. Solutions of aromatic bases such as phenylenediammonium salts are often used for eluents in conjunction with UV-vis detection (43). Negative peaks again result (decreased absorbance) as the sample cations pass through the detector.

The separation of divalent metal cations can be extended to include several of the transition and rare earth metal cations by incorporating a weakly complexing organic acid such as tartrate, citrate, oxalate, pyridine-2,6-dicarboxylate, or hydroxyisobutyrate in the eluent $(44,45)$. Heavy metals can also be separated by ion chromatography followed by post-column derivatization reaction with a chromogenic ligand for optical detection. This idea was introduced by Sickafoose in 1971 (46) and 
is still practiced $(47,48)$.

In another approach, transition metals can be analyzed by ion chromatography following preconcentration on a column containing a chelating functional group such as iminodiacetate $(49,50)$.

Atomic absorption and emission techniques are commonly used for the measurement of alkali and alkaline earth metal cations in samples such as brines. However, ion chromatography is becoming widely accepted as an alternative method $(51,52)$. The differing ion-exchange affinities of the alkali and alkaline earth metal cations has traditionally made it difficult to find simple isocratic elution conditions to effectively separate both types of cations in a reasonable time period. However, certain isocratic eluents have been shown to allow simultaneous separation of these two groups of cations. Eluents containing inorganic species such as copper (53), cobalt (54), and cerium (55), and complexing mobile phases such as diaminopropionic acid (56), citric acid, and ethylenediaminetetraacetic acid (EDTA) (57) have been used.

Recently, Kolla, Kohler, and Schomburg (58) introduced a unique column chemistry that made possible the simultaneous separation of alkali and alkaline earth metal cations using a mildly acidic complexing eluent and unsuppressed conductivity detection. The column, which is now commercially available, is packed with a poly(butadiene-maleic acid)-coated silica stationary phase. This work was expanded to study several weak-acid eluting agents for the separation of up to 18 cations (59). Results obtained on this type of column showed excellent agreement with those obtained by inductively coupled plasma-atomic emission spectroscopy (ICP-AES) (60). 
Research presented in Paper I of this dissertation was a study of cation separations on self-prepared weak-acid resins. The resins were easily synthesized and carry the exchange group directly on the crosslinking benzene ring or on a short spacer arm from the ring of a macroporous PS-DVB resin. The resin starting material was similar to those commonly used for ion chromatography. The synthesis and general properties of two carboxylic acid resins are described. Eluents containing ethylenediammonium cations plus various complexing organic acids were investigated. Since the carboxylic acid functional groups are weak acids, the effect of $\mathrm{pH}$ on the retention times of metal cations was also studied.

\section{Ion-Exclusion Chromatography of Organic Acids}

Ion-exclusion chromatography (IEC) was first described by Wheaton and Bauman in 1953 (61). Since that time, IEC has gained popularity as a technique that is complementary to ion-exchange chromatography. A number of excellent reviews are available that describe the separation principles, columns, eluents, and detection methods for determination of carboxylic acids, alcohols, sugars, and other hydrophilic compounds (62-74).

Early IEC separations were performed on cation-exchange columns using water as an eluent and conductometric detection. Separation of solute species was based on the fraction of the sample species that existed in the ionic form and in the molecular form. Completely ionized species (anionic form) would be repelled by the negatively charged sulfonic acid groups of the cation-exchange resin and would pass through the column with the void volume of the system. Neutral samples, on the other hand, could 
penetrate and partition into the cation-exchange beads. These samples would then elute at the volume equal to the void volume plus the volume of water occluded in the beads. Partially ionized compounds would elute at retention volumes between these two limits. Due to the limited size of this elution volume "window", peak capacities tended to be rather small.

In 1978, work by Turkelson et al. (75) began what is now referred to as "Modern IEC". A dilute mineral acid was employed as an eluent instead of water. The low $\mathrm{pH}$ of this eluent would repress ionization of the sample acids so that they would exist mostly in the molecular form. Retention of the solutes could now exceed the previous theoretical maximum because of other column interactions. These additional interactions include hydrogen bonding or, more importantly, hydrophobic interactions which are important for sorption in the reversed-phase systems.

Detection methods commonly used include UV absorbance, for sample species that contain an appropriate UV-absorbing chromophore, or a more general refractive index detector. In one case, the use of a potentiometric detector was illustrated and the mechanism of response was described (76). The use of an acidic eluent makes the use of conductivity detection comparatively insensitive since the background conductance of the eluent is quite high.

Many authors have developed supressor-based detection schemes to overcome this problem and allow the use of conductivity detection.

Pohlandt (77) described the preparation and use of a Ag-loaded cation-exchange column for supressed conductometric detection. IEC was used to separate carboxylic 
acids from other strong-acid anions with a dilute solution of hydrochloric acid as an eluent. The measurement was made possible by precipitation-adsorption of the eluent anions on the $\mathrm{Ag}$-loaded resin.

Tanaka and Fritz (78) illustrated the use of a two-stage conversion system to increase detection sensitivity. Very weak acids $\left(\right.$ e.g. $\left.\mathrm{CO}_{2}\right)$ were separated using water as an eluent. The eluate was then passed through two sequential ion-exchange columns in which $\mathrm{HX}$ was converted to $\mathrm{KOH}$ (or $\mathrm{NaOH}$ ). This would yield an enhanced conductivity signal.

Two authors, Murayama (79) and Haginaka et al. (80) described a cationexchange, hollow-fiber suppressor system to enhance the detection of carboxylic acids after separation by IEC. Neutral salt or alkaline solutions were employed as enhancers which were pumped on the outside of the suppressor tube. The $\mathrm{pH}$ of the eluate rose through the suppressor system to enhance the peak height and to suppress the background

Okada (81) used a different supression technique that was particularly effective with highly acidic eluents which permit a large number of carboxylic acids to be resolved by IEC. Hydriodic acid was used as an eluent and reacted postcolumn with hydrogen peroxide to produce non-ionic, non-conducting iodine. The peak heights of some weak acids were up to an order of magnitude higher using this method.

Okada and Dasgupta (82) described a technique for optical detection of weak acids in the $\mathrm{pK}_{\mathrm{a}}$ range of 4.5-9.5. The strong-acid eluent was subjected to exchange with $\mathrm{Na}^{+}$in a membrane supressor, resulting in a neutral $\mathrm{pH}$ effluent. In contrast, 
weak acids produce alkaline salts. This process was detected optically via an acid-base indicator introduced through a membrane. Even with $10 \mathrm{mM}$ nitric acid as an eluent, detection limits of $50 \mathrm{pmol}$ were possible.

Several authors have shown the effective use of other eluent systems. Tanaka and Fritz (83) found that a solution of either benzoic or succinic acid was an effective eluent for the separation of aliphatic carboxylic acids with conductivity detection. The background conductance of these eluents was much lower than the strongly acidic eluents commonly used and permitted a more sensitive detection of the sample acids.

Okada (84) used a mobile phase containing mixed ionic and non-ionic surfactants for the separation of carboxylic acids. Advantages resulted from the formation of mixed micelles of the two surfactants, and the analysis time was reduced by partitioning of the analytes into the micelles.

Botte and Joergensen (85) investigated the use of 5-sulfosalicylic acid as an eluent for the IEC separation of weak acids with supressed conductometric detection. The supressed background conductance was decreased by $25 \%$ from that found for sulfuric acid and the sensitivity was improved accordingly.

Haddad et al. $(86,87)$ examined a range of eluents to manipulate the selectivity of retention in IEC when applied to the separation of common water-soluble carboxylic acids. Eluents included water and dilute solutions of sulfuric acid, phosphoric acid, ptoluenesulfonic acid, methanesulfonic acid, and benzoic acid. In addition, Bcyclodextrin was introduced into some eluent systems as a means of reducing the retention times of aromatic solutes through the formation of inclusion complexes with 
the B-cyclodextrin. This provided an additional parameter for control of solute retention.

The effects of carbonic acid were investigated in two opposite approaches. In one case, organic acids were separated by IEC using a carbonic acid solution as an eluent. This permitted conductometric detection of ionic species without a supressor as a post-column reactor. The proposed method was applicable to separation of organic acids having a $\mathrm{pK}_{\mathrm{a}}$ over 3.5 (88). An alternative approach was to remove atmospheric carbon dioxide by bubbling nitrogen through an eluent used for simultaneous determination of bicarbonate and carboxylates in rainwater by IEC. Displacement of the interfering carbon dioxide increased peak heights of the weak acids by $13-20 \%(89)$.

Ion-exclusion chromatography has proven to be a useful technique for a wide variety of applications. Weak acids are routinely determined in beverages $(90,91)$, foods (92), sugar process juices (93), rainwater (94), soils (95), atmospheric gas $(96,97)$, and steam generator blowdown (98). Methods have been developed for the separation of aromatic acids $(99,100)$, and hydroxy acids were separated from inorganic solids in wood-pulping liquor samples (101).

IEC is also utilized for other hydrophilic compounds such as alcohols in i.v. solutions (102). Alkanolamines were separated on an anion-exchange column using an aqueous eluent containing glycerin to reduce tailing (103). Sugars and related compounds were separated on cation-exchange columns loaded with alkaline earth and transition metals (104). 
Carboxylic acids and inorganic acids were separated by coupling nonsuppressed ion-exchange chromatography with ion-exclusion chromatography. The sample was injected into the ion-exclusion column, which separated the weak-acid anions. The void volume was collected on-line and injected into an anion-exchange column, which separated the strong-acid anions (105).

Traditionally, ion-exclusion columns contain a highly sulfonated microporous (gel-type) cation-exchange resin and are quite large $(30 \mathrm{~cm} \times 7.8 \mathrm{~mm})$. The reason for the large column was based on early theoretical considerations. As previously discussed, most sample species elute in the narrow elution volume "window" between interstitial volume and interstitial volume plus internal bead volume. The larger columns could hold more resin, thus increasing the total column volume and the directly related peak capacity.

Use of macroporous resins for separations by IEC is gaining attention. Early work by Lehotay and Traiter (106) studied the effect of the degree of crosslinking on the efficiency of the separation and also the volume distribution coefficients of sample acids. In most instances, the values determined for the volume distribution coefficients were found to decrease as the degree of crosslinking increased. Successful separations on highly-crosslinked macroporous resins, however, would indicate a different mechanism of separation than that previously accepted.

Lee and Lord (107) evaluated a series of macroporous stationary phases for the separation of the simple aliphatic carboxylic acids. They found that the acids were more strongly retained by the partially sulfonated materials than by the more 
hydrophobic underivatized reversed-phase materials. Walser (108) conducted further studies with these same stationary phases. A mathematical model was used to describe selectivity. Comparison of separation characteristics with other commercial columns showed the separation mechanism to be controlled by hydrophobic interaction, hydrogen bonding, and ion exclusion.

In research presented in paper II of this dissertation, lightly sulfonated or carboxylated macroporous PS-DVB resins were used for the separation of weak acids and other small polar cc.npounds using eluents of dilute sulfuric acid. The predominant mechanism of separation was determined to be partitioning of sample species between the primarily aqueous eluent and the resin matrix. The use of organic solvents for controlling retention of solute species with a more hydrophobic nature was explored. A data acquisition system was used to correct a rising baseline encountered with gradient elution and UV detection.

\section{Effect of Eluent Modifiers on HPLC Separations}

Retention behavior in liquid chromatography is a function of the strength of a solute's interaction with both the mobile and stationary phase. Once an appropriate stationary phase is chosen, selectivity is adjusted by modifying the mobile phase. A number of preferred organic solvents have evolved for this purpose.

The solvent properties of greatest chromatographic interest are strength and selectivity. Strength, in this case, is synonymous with polarity. In reversed-phase HPLC separations, a certain percentage of an organic solvent is incorporated into an aqueous eluent. In doing so, the polarity of the mobile phase is decreased and the eluting 
power or strength is increased accordingly. Selectivity has been described as "the ability of a given solvent to selectively dissolve one compound as opposed to another, where the polarities of the two compounds are not obviously different" (109).

For many practitioners of HPLC, selecting a mobile phase is still a matter of trial and error. Snyder $(109,110)$ has described a useful scheme for classifying common solvents according to their polarity or chromatographic strength and their selectivity based on the principle intermolecular forces responsible for their properties. A selectivity triangle for solvents was constructed and offered a structured methodology for solvent selection. In many instances, mixtures of solvents as a mobile phase are used rather than a single pure solvent. Binary solvent mixtures provide a simple means to control solvent strength but are limited in controlling selectivity. Choosing a solvent of similar strength but with different selectivity can improve peak resolution. Use of ternary or quarternary solvent mixtures often make it possible to fine tune solvent selectivity while maintaining a constant solvent strength (111-113).

Several authors have investigated the effect of incorporating mobile phase modifiers of various functional groups. Alcohol modifiers were used in nonpolar mobile phases, such as hexane or heptane, for the chromatographic separation of several bi- and trifunctional solutes (114) and fat-soluble vitamins in vegetable oil compounds (115).

Many chiral separations were performed using similarly modified eluent systems (116-119). In most cases, it was found that the steric structure of the alcohol modifier had an effect on the capacity factor and stereoselectivity. Results indicated that the 
alcohols would compete with the solutes for chiral and achiral binding sites and that the steric bulk around the hydroxyl moiety of the modifiers played a role in this competition. In a related study, the adsorption of propanol and acetonitrile on the chiral column was measured and monolayers were found at modifier concentrations of $10 \%$ and $15 \%$ respectively $(120)$.

Beta-cyclodextrin was used as a mobile phase in the reversed-phase separation of polycyclic aromatic hydrocarbons $(121,122)$. Bonded cyclodextrin was used as a stationary phase for a similar separation (123). In both cases, the addition of certain alcohol modifiers to the mobile phase promoted the formation of cyclodextrin inclusion complexes with the polynuclear species by forming ternary complexes.

Khaledi (124) studied selectivity for homologous series in reversed-phase HPLC using micelles and organic solvents as the modifiers to an aqueous mobile phase. With the hydro-organic mobile phase, selectivity depended only on the eluent composition. For micellar eluents, however, selectivity was a function of the solute type and its location in/on the micelle.

Tomasella et al. (125) examined the role of organic modifiers in micellar liquid chromatography. They calculated the change in the mobile-stationary phase equilibrium caused by addition of the organic modifier and described the resulting change in retention mechanism.

A neutral, coordinatively-unsaturated metal complex was introduced as a mobile phase modifier for HPLC of amino alcohcls (126). Interaction with the metal complex and the subsequent increase in solute retention was shown to be dependent on steric 
dipole and solvation effects.

Naleway and Hoffman (127) tested different carboxylic acids as mobile phase modifiers. They studied the effect of acid chain length, $\mathrm{pH}$, and eluent composition on retention. The retention of both neutral and positively charged compounds was influenced by the dissociation equilibrium of the carboxylic acid in the mobile phase. The inflestion in the retention of neutral and charged soivtes is $\mathrm{pH}$ was changed occurred at the $\mathrm{pK}_{\mathrm{a}}$ of the acid in the mobile phase.

Heldin et al. (128) studied the separation of enantiomers of acids, es: $s$, and amino alcohols of moderate hydrophobicity using (2R,3R)-dicyclohexyl tartrate (DCHT) in phosphate buffer as a mobile phase and porous graphitic carbon as a stationary phase. DCHT was found to have a high affinity for the graphitic carbon phase with this mobile phase. It seemed to be adsorbed on the support as a monolayer, since changes in the concentration of the mobile phase had only a minor influence on the amount adsorbed and addition of acetonitrile to the mobile phase decreased the amount of DCHT adsorbed on the carbon support.

Cheong and Carr (129) measured surface tension data for aqueous binary mixtures of several organic solvents. The sequence of eluotropic strength based on the solvophobic theory in reversed-phase HPLC was listed as DMSO $<\mathrm{MeOH}<\mathrm{MeCN}$ $<$ THF $<$ iso-PrOH $<$ t-BuOH.

Michels and Dorsey (130) measured the retention behavior of a variety of solutes using a homologous series of normal alcohols as organic modifiers in hydroorganic mobile phases. The results imply that a systematic change in the extent of 
solvation of the stationary phase occurred with respect to the size of the organic modifier.

Lau and Simpson (131) proposed a way to control variation in separation characteristics of different columns. The effect of chain length of a homologous series of alkanol modifiers in different solvent mixtures of methanol/water was studied on commercially available columns. Columns could be modified to present the same surface area/volume for interaction with solute molecules and hence to obtain equivalent separations.

Research presented in paper III of this dissertation studied the effect of mobile phase modifiers of various functionalities on the chromatographic behavior of both ionic and neutral solute species. Small straight-chain alcohols added to an aqueous mobile phase were found to have a dramatic effect on the chromatographic behavior of alkane carboxylic acids. The alcohols were believed to coat the resin surface and establish a dynamic equilibrium between the mobile and stationary phases. This altered the hydrophilicity of the resin surface. In a sense, this permitted fine-tuning of the siationary phase capacity behavior. These eluent systems have a very low background conductance, thus allowing very sensitive conductometric detection. The presence of carbonic acid (from atmospheric carbon dioxide) in the eluent was determined to play a role in the retention mechanism of the weak-acid species. The use of larger straight-thain alcohols as modifiers in a water/acetonitrile mobile phase was investigated for the separation of neutral organic species. Finally, a 12-carbon alcohol was semi-permanently coated on the PS-DVB resin. This treated column was 
used for separation of alkane carboxylic acids using a purely aqueous eluent and conductometric detection. 
PAPER I. ION CHROMATOGRAPHY OF METAL CATIONS ON CARBOXYLIC ACID RESINS 


\section{ION CHROMATOGRAPHY OF METAL CATIONS ON CARBOXYLIC ACID RESINS}

John Morris and James S. Fritz

Ames Laboratory - U.S. Dept. of Energy and Department of Chemistry, Iowa State University Ames, IA 50011, U.S.A.

Reproduced with permission from the Journal of Chromatography, 1992, 602, 111-117. Copyright 1992 Elsvier Science Publishers B.V. 


\section{SUMMARY}

The goal of the present work is to study cation separations on weak-acid resins that are easily synthesized and carry the exchange group on the crosslinking benzene ring of the resin or on a short spacer arm from the ring. The synthesis and general properties of two carboxylic acid resins are described. A higher exchange capacity is required for effective ion chromatographic separations than that needed for sulfonated resins. A number of excellent separations are reported using eluents containing ethylenediammonium cations plus a weak complexing agent. The eluent $\mathrm{pH}$ has a considerable effect on retention times of metal ions. As the $\mathrm{pH}$ becomes more acidic, the weak-acid functional group of the resin loses its ionic capacity and the resin exchange capacity is decreased. Retention times of metal ions are reduced accordingly. 


\section{INTRODUCTION}

Perhaps the best method for separating $2^{+}$and $3^{+}$metal cations by ion chromatography is through the use of complexing eluents. Sevenich and Fritz (1) obtained excellent separations using an ethylenediammonium tartrate eluent in conjunction with a conductivity detector. Further selectivity is possible with the addition of a second chelating reagent (such as EDTA) to the sample only (2). Others have obtained excellent separations of various metal ions using eluents containing tartrate, citrate, oxalate, 2-hydroxyisobutyrate, or salts of pyridyldicarboxylic acid (PDA) $(3-7)$.

Virtually all ion chromatographic separations of metal cations have been carried out on various strong-acid ion exchangers containing the sulfonic acid group. These strongly acidic ion exchangers maintain complete ionic capacity over a large $\mathrm{pH}$ range of 2-12. A weak-acid exchanger, such as the carboxylic acid, loses its ionic capacity as the $\mathrm{pH}$ goes below the $\mathrm{pK}_{\mathrm{a}}$ of the functional group. The $\mathrm{pH}$ greatly affects the separation of the metal cations thus adding an additional parameter for optimization. Kolla, Kohler, and Schomburg (8) prepared an efficient carboxylic acid resin by coating

a polybutadiene-maleic acid resin onto the surface of $5 \mu \mathrm{m}$ porous silica. Using this resin, they obtained simultaneous separations of monovalent and divalent cations.

The preparation and use of two new carboxylic acid resins are described in the present research. Both resins are prepared by simple Friedel-Crafts addition reactions. One has the carboxyl group attached to the benzene ring of a spherical polystyrene- 
divinylbenzene resin via a spacer arm of three carbon atoms. The carboxyl group is attached directly to the benzene ring in the other resir. Excellent ion chromatographic separations of metal ions are possible using these resins in conjunction with any of several complexing eluents. 


\section{EXPERIMENTAL}

\section{Apparatus}

The chromatographic system consisted of several components. An Eldex model AA-94 pump (Eldex Labs, Inc., Menlo Park, CA) set to deliver eluent at a flow rate of $1.0 \mathrm{ml} / \mathrm{min}$, a 7125 Rheodyne injector (Rheodyne, Berkeley, CA) equipped with a 10- $\mu$ l loop, a Wescan ICM II ion analyzer (Wescan Instruments, Santa Clara, CA) with conductivity detection at a constant temperature, a Fisher Recordall series 5000 recorder (Fisher Scientific/Instrument Lab, Itasca, IL), and a Hitachi D-2000 integrator (EM Science, Cheryy Hill, NJ) were used for all HPLC separations. A Shandon HPLC packing pump (Shandon Southern, Sewickley, PA) was used for column packing.

\section{Reagents and chemicals}

The spherical PS-DVB resins (Sarasep, Santa Clara, CA) used in this experiment consisted of two sizes; $10-\mu \mathrm{m}$ resin with $80 \AA$ pore size and $415 \mathrm{~m}^{2} / \mathrm{g}$ surface area, and $5-\mu \mathrm{m}$ resin with similar pore size and surface area. The resin was washed with water, acetonitrile, and methanol, and then dried.

The reagents and solvents used for the derivatization reactions were reagent grade. Stock solutions of the metals were prepared from reagent grade chloride salts. The concentrations of metal ions in the salt solutions injected ranged from $1.2 \times 10^{-4}$ to $1.1 \times 10^{-3} \mathrm{M}$. Eluents were prepared daily using reagent grade solutions of ethylenediamine and the specified organic acid. A Barnstead Nanopure II system (Sybron Barnstead, Boston, MA) was used to further deionize distilled water for all eluents and sample mixtures. Adjustments in $\mathrm{pH}$ were made with reagent grade 
solutions of sodium hydroxide and hydrochloric acid.

\section{Synthesis procedures and capacity determination}

The carboxylic acid functional groups were introduced into the benzene ring of PS-DVB resins by the following procedures:

(1) $-\mathrm{COCH}_{2} \mathrm{CH}_{2} \mathrm{CO}_{2} \mathrm{H}$ derivative (Resin I). Mix $2.5 \mathrm{~g}$ of resin with $3.6 \mathrm{~g}$ of succinic anhydride and $50 \mathrm{ml}$ of tetrachloroethane in a $100-\mathrm{ml}$ round-bottomed flask. Stir for 15 minutes, then add $10.7 \mathrm{~g}$ of anhydrous aluminum chloride and reflux at $45^{\circ} \mathrm{C}$ for 24 hours. Pour the product into a methanol/ice solution to quench the reaction. Collect the resin by filtration, wash with methanol, $1 \mathrm{M} \mathrm{HCL}$ and water, then dry. The exchange capacity was found to be $0.60 \mathrm{meq} / \mathrm{g}$ by acid-base titration of the carboxylic acid.

The capacity determination was as follows. An accurate amount of resin was weighed and an accurate volume of a standardized $\mathrm{NaOH}$ solution was added. This was shaken for several hours in a sealed vial to ensure complete neutralization of the resin weak-acid functionality. The resin was then isolated by filtration and washed several times to remove all excess $\mathrm{NaOH}$. The entire wash solution was collected and titrated with a standardized acid. The reported capacity was the result of three successive trials.

(2) $-\mathrm{CO}_{2} \mathrm{H}$ derivative (Resin $\mathrm{II}$ ). Mix $2.5 \mathrm{~g}$ of resin with $8.0 \mathrm{ml}$ of phenyl chloroformate and $50 \mathrm{ml}$ of tetrachloroethane in a $100-\mathrm{ml}$ round-bottomed flask. Stir for 15 minutes, then add $10.0 \mathrm{~g}$ of anhydrous aluminum chloride and reflux at $100^{\circ} \mathrm{C}$ for 4 hours. Pour the product into a methanol/ice solution. Isolate the resin, wash 
with methanol, then dry. Hydrolysis of the ester is accomplished by refluxing the resin for 1 hour in a $1 M \mathrm{NaOH} /$ ethanol solution. Collect the resin, wash with methanol, $1 M \mathrm{HCl}$ and water, then dry. The exchange capacity was found to be $0.39 \mathrm{meq} / \mathrm{g}$ by acid-base titration of the carboxylic acid.

The Friedel-Crafts reactions used in the preparation of Resins I and II are shown in Figure 1.

\section{Column packing procedure}

The PS-DVB resin and its various derivatives were packed into stainless steel HPLC columns. A small amount (about $2 \mathrm{~g}$ ) of the dried resin was suspended in $30 \mathrm{ml}$ of methanol/water $(50 / 50)$. The suspension was then packed into the stainless steel column by the Shandon HPLC packing pump. The packing pressure was set at 3000 psi. The packing eluent was methanol/water $(50 / 50)$ and was degassed by helium purging prior to use. The stainless-steel columns had a $4.6 \mathrm{~mm}$ inner diameter and lengths of $5-15 \mathrm{~cm}$. 
Figure 1. Friedel-Crafts derivatization reactions for the preparation of Resins I and II 

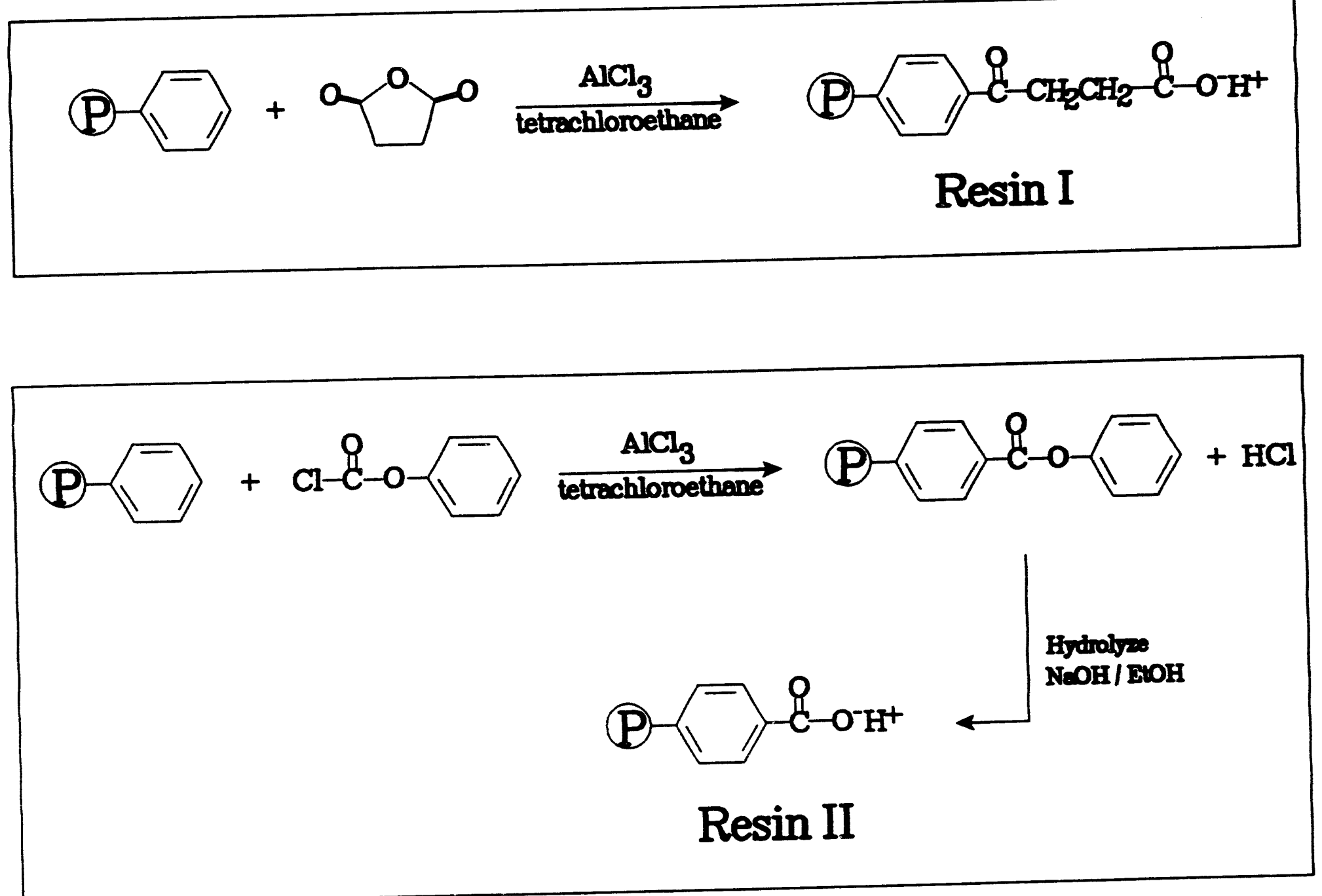


\section{RESULTS AND DISCUSSION}

\section{Preparation of Resins}

Resin $I$ was prepared by reacting a porous, crosslinked polystyrene resin with succinic anhydride in a Friedel-Crafts reaction. The chemical structure of the derivatized resin can be written as $\mathrm{P}-\mathrm{C}_{6} \mathrm{H}_{4} \mathrm{COCH}_{2} \mathrm{CH}_{2} \mathrm{CO}_{2} \mathrm{H}$. Resin II was prepared by reacting the polystyrene resin with phenyl chloroformate in a Friedel-Crafts reaction, followed by hydrolysis to the carboxylic acid. The chemical structure can be written as $\mathrm{P}_{-} \mathrm{C}_{6} \mathrm{H}_{4} \mathrm{CO}_{2} \mathrm{H}$. Coppock (9) showed that phenyl chloroformate was required in the Friedel-Crafts reaction of aromatic hydrocarbons to obtain the expected aryl ester of the aromatic hydrocarbon. Alkylation of the hydrocarbon occurs with the use of alkyl chloroformates.

At first, Resin $I$ was prepared under very mild reaction conditions to give a carboxylic acid capacity $<0.1 \mathrm{meq} / \mathrm{g}$, which is within the range that is widely used for the sulfonic acid resins used in ion chromatography. However, this carboxylic acid resin failed to give any useful separations. Another batch of Resin $I$ was prepared under different reaction conditions to give a resin of much higher carboxylic acid capacity $(0.60 \mathrm{meq} / \mathrm{g})$. This resin (and others of similar capacity) was used very successfully for ion chromatographic separations.

\section{Optimization of Separation Conditions and Effect of pH}

Earlier work with columns of sulfonated polymeric resins demonstrated that good separation of several divalent metal ions could be obtained using weakly complexing eluents of ethylenediammonium tartrate $(1,2)$. Almost all of the present 
work was done with carboxylic acid Resin $I$. Separations of several divalent metal ions were obtained using eluents containing the ethylenediammonium $2^{+}$cation and one or twc of the following complexing anions: citrate, pyridine-2,6-dicarboxylate (PDA), oxalate, hydroxybutyrate, and tartrate. The first three of these were found to give the best separations. The ethylenediammonium $2^{+}$cation acts as a "pusher" to move the divalent metal ions down the column. The complexing anions act as "pullers" to aid the separation of metal ions based on the formation constant of each metal ion with that particular anion.

Experiments designed to optimize separation conditions showed that the ethylenediammonium cation and a complexing anion were both needed in the eluent. Broad peaks and few separations were obtained using the ethylenediammonium cation with only a non-complexing anion. Addition of any of the complexing anions tightened the chromatographic peaks considerably. However, very poor separations were obtained when the eluent contained a complexing anion in conjunction with a monovalent cation such as sodium. The presence of ethylenediammonium, or some other divalent cation, seemed to be necessary. Using an eluent consisting of ethylenediammonium and any of the mentioned complexing anions, there is a noticeable later eluting system peak at lower $\mathrm{pH}$ values ( $\mathrm{pH} 4.0$ to 4.8 ). The retention time of this system peak is well beyond that of any of the metal ions in a mixture and adds no interference. The majority of work reported here was at $\mathrm{pH} 5.0$ and higher where the system peak is no longer noticed and can be ignored. The system peak never appeared in later chromatograms when doing successive injections. 
The effect of varying $\mathrm{pH}$ was investigated using the ethylenediammonium oxalate eluents. Figures 2 and 3 show the results of plotting $\log$ of the adjusted retention time versus $\mathrm{pH}$ for some selected divalent metal ions. Further examination of the results in Table I show the logarithm of adjusted retention time to be a linear function of $\mathrm{pH}$ ( $t_{\mathrm{R}}^{\cdot}$ increasing as the $\mathrm{pH}$ assumes a higher value). The metal ions studied fell into two distinct groups, one with a slope very close to 0.5 and the other with a slope of approximately 1.0. We have no explanation for these differences in slope except that some of the metal ions might form metal hydrogen oxalate complexes and the others simple metal oxalate complexes.

The $\mathrm{pH}$ study was conducted in the $\mathrm{pH}$ range where oxalate was predominantly in the 2 form. A complexing anion of a weaker acid would be converted more completely to the fully deprotonated form as the $\mathrm{pH}$ is increased. This would increase its chelating ability and could therefore lead to lower $t_{R}^{\prime}$ values with increased $\mathrm{pH}$.

Increasing the concentration of complexing anion in the eluent leads to more complete complexation of metal ions and thus to lower $t_{R}^{\prime}$ values. The value of $t_{R}^{\prime}$ for any given metal ion seems to be determined primarily by the formation constant of the metal-anion complex, although this is modified by the affinity of the resin for the uncomplexed metal cation. The importance of metal-anion formation constants is demonstrated by the pyridine-2,6-dicarboxylate (PDA) anion. PDA has a rigid planar structure which forms very strong complexes with transition metals leading to fast chromatographic elution. Alkaline earths, however, form weaker complexes allowing excellent chromatographic separation. The order of elution of metal ions for all 
Figure 2. Plot of $\log t_{R}$ ' versus $\mathrm{pH}$ for divalent metal ions exhibiting a slope of approximately 0.5 


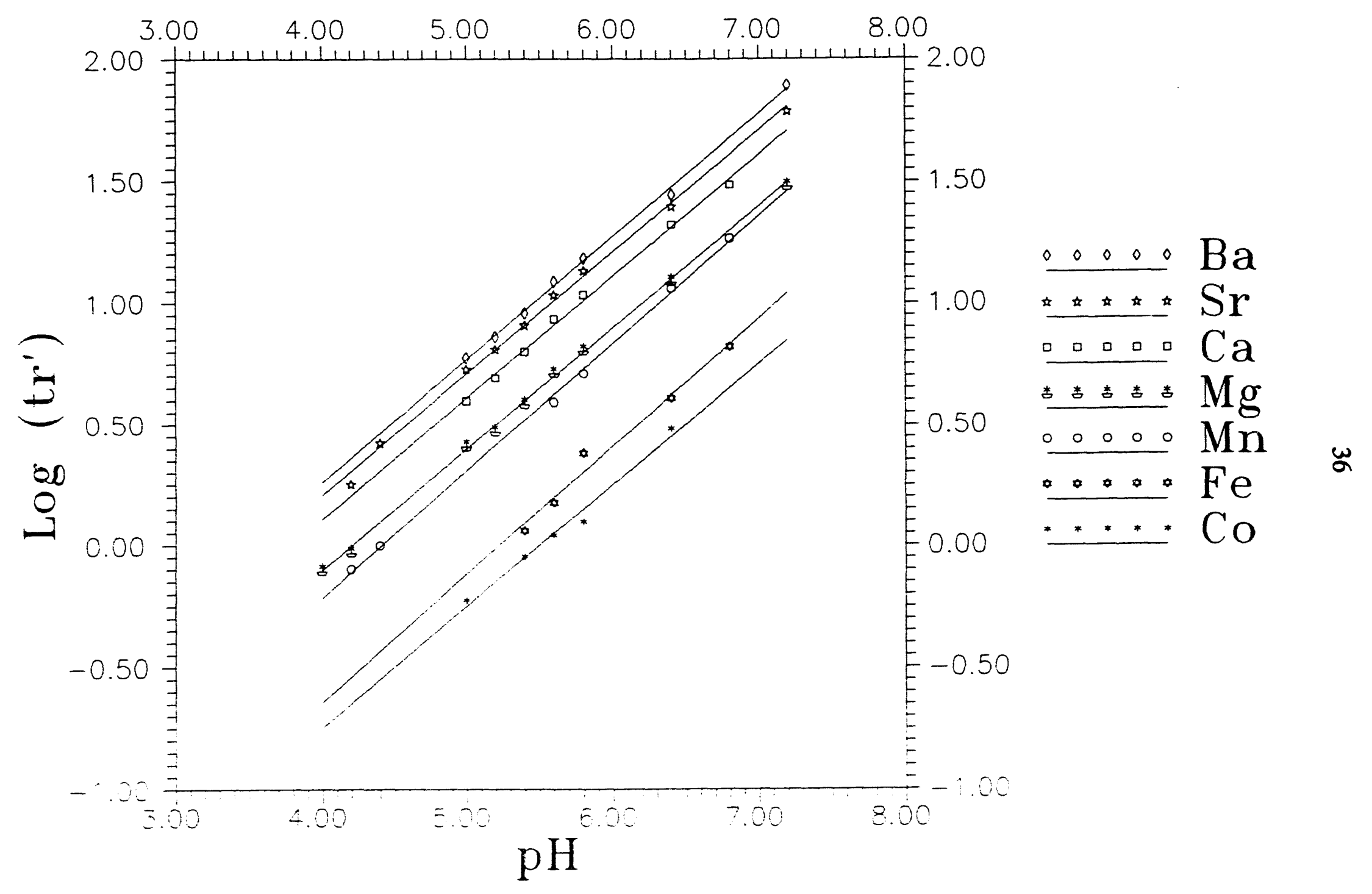


Figure 3. Plot of $\log t_{R}$ ' versus $\mathrm{pH}$ for divalent metal ions exhibiting a slope of approximately 1.0 


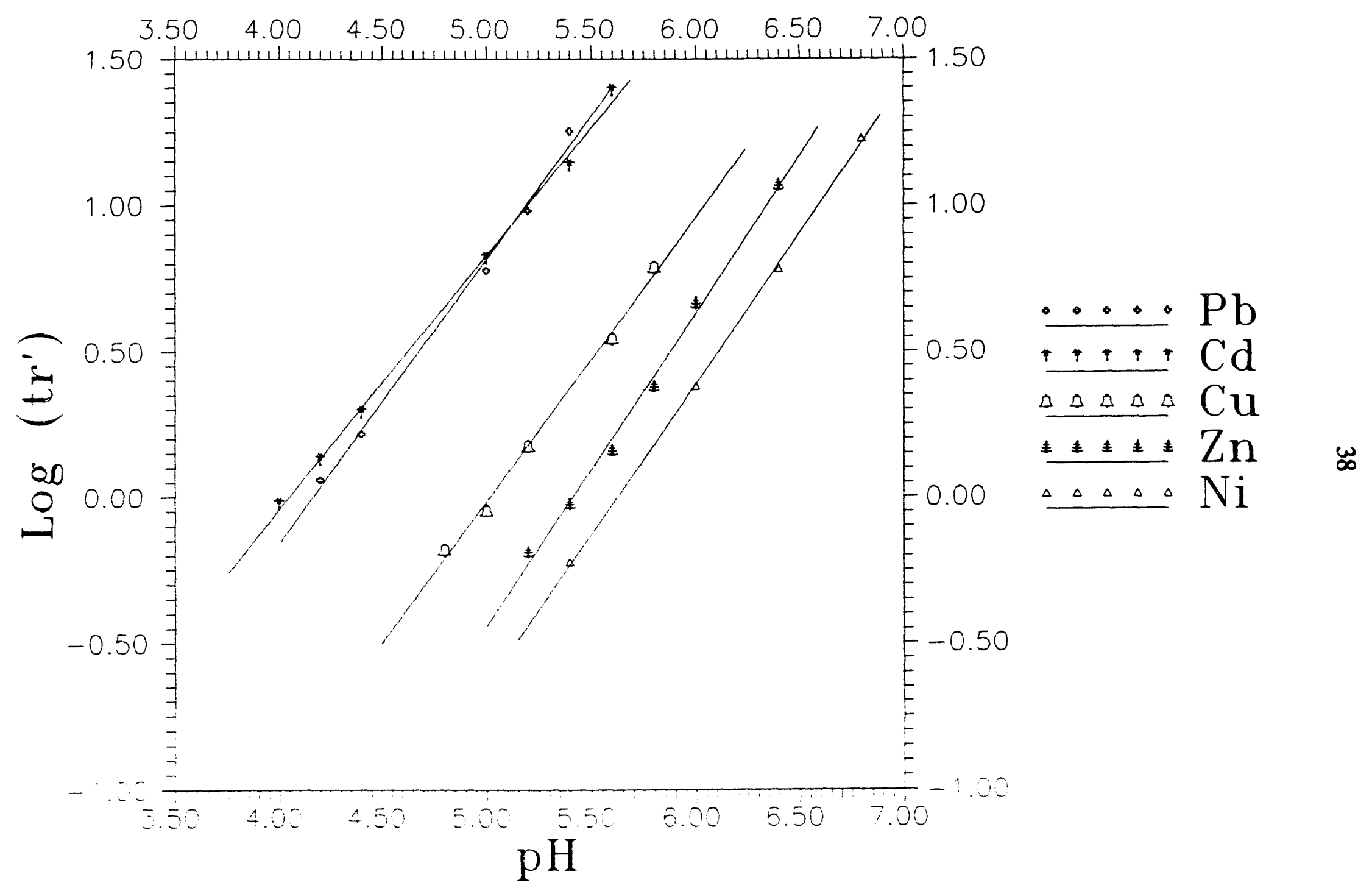


Table I. Linear regression data for plots of $\log t_{R}^{\cdot}$ vs. pH using an ethylenediammonium oxalate eluent

\begin{tabular}{||ccccc||}
\hline Metal ion & $\mathrm{pH}$ range & Slope & Intercept & $\begin{array}{c}\text { Correlation } \\
\text { coefficient }\end{array}$ \\
\hline $\mathrm{Ni}^{++}$ & $5.4-6.8$ & 1.03 & -5.80 & 0.9994 \\
$\mathrm{Co}^{++}$ & $5.0-6.8$ & 0.50 & -2.74 & 0.9822 \\
$\mathrm{Zn}^{++}$ & $5.0-6.4$ & 1.07 & -5.80 & 0.9944 \\
$\mathrm{Fe}^{++}$ & $5.4-6.8$ & 0.53 & -2.75 & 0.9802 \\
$\mathrm{~Pb}^{++}$ & $4.0-5.4$ & 0.98 & -4.06 & 0.9948 \\
$\mathrm{Cu}^{++}$ & $4.2-5.8$ & 0.97 & -4.88 & 0.9952 \\
$\mathrm{Cd}^{++}$ & $4.0-5.6$ & 0.87 & -3.52 & 0.9976 \\
$\mathrm{Mn}^{++}$ & $4.2-7.2$ & 0.52 & -2.31 & 0.9986 \\
$\mathrm{Mg}^{++}$ & $4.0-7.2$ & 0.50 & -2.10 & 0.9991 \\
$\mathrm{Ca}^{++}$ & $4.0-7.2$ & 0.50 & -1.89 & 0.9962 \\
$\mathrm{Sr}^{++}$ & $4.0-7.2$ & 0.51 & -1.86 & 0.9977 \\
$\mathrm{Ba}^{++}$ & $4.0-7.2$ & 0.51 & -1.76 & 0.9980 \\
\hline \hline
\end{tabular}


complexing anions studied was found to be that of decreasing complex formation constants (see Table II).

An earlier study with complexing eluents in conjunction with sulfonated resins showed that $\log t_{R}^{\prime}$ is inversely proportional to $\log \alpha_{M}$, where $\alpha_{M}$ is the concentration ratio of free metal cation to the total metal cation in solution (1). This paper also showed that $\log t_{R}^{\prime}$ is a linear function of the logarithm of the ethylenediammonium concentration in the eluent.

\section{Citrate systems}

An eluent containing $1.0 \mathrm{mM}$ ethylenediammonium ion and slightly lower concentrations of citrate at pH 5.4 was found to give some useful separations. Keeping the ethylenediammonium concentration and the $\mathrm{pH}$ constant, the citrate concentration of the eluent was varied from 0.2 to $0.6 \mathrm{mM}$ in $0.1 \mathrm{mM}$ increments. Poor separations were obtained at $0.2 \mathrm{mM}$ and $0.6 \mathrm{mM}$ citrate; the best separation was obtained at 0.3 $m M$ citrate.

Figure 4 shows the separation of several metal ions at $0.3 \mathrm{mM}$ citrate. The $\mathrm{Ni}^{++}$peak is covered by the injection peak. The $\mathrm{Co}^{++}$and $\mathrm{Zn}^{++}$peaks are well resolved even though the ratio of the logarithmic citrate formation constants is only 1.03 (Table II). The $\mathrm{Ca}^{++}$and $\mathrm{Sr}^{++}$peaks (ratio of log constants $=1.13$ ) and $\mathrm{Sr}^{++}$ and $\mathrm{Ba}^{++}$peaks (ratio of $\log$ constants $=1.10$ ) are resolved but the resolution of the $\mathrm{Mg}^{++}$and $\mathrm{Ca}^{++}$peaks is poor, probably due to the very small ratio of their logarithmic formation constants $(=1.02)$. 
Table II. Logarithms of formation constants of selected metal complexes

\begin{tabular}{|l|lllllllll||}
\hline & \multicolumn{7}{|c||}{ Log formation constants } & & \\
Reagent acid & $\mathrm{Cu}^{++}$ & $\mathrm{Ni}^{++}$ & $\mathrm{Co}^{++}$ & $\mathrm{Zn}^{++}$ & $\mathrm{Mn}^{++}$ & $\mathrm{Mg}^{++}$ & $\mathrm{Ca}^{++}$ & $\mathrm{Sr}^{++}$ & $\mathrm{Ba}^{++}$ \\
\hline \hline Citric & 5.60 & 5.11 & 4.83 & 4.70 & 3.70 & 3.25 & 3.18 & 2.81 & 2.55 \\
$\begin{array}{l}\text { Pyridine-2,6- } \\
\text { dicarboxylic } \\
\text { Oxalic }\end{array}$ & 8.80 & 6.60 & 6.35 & 6.43 & 4.70 & 2.02 & 4.30 & 3.50 & 3.13 \\
\hline
\end{tabular}




\section{2}

Figure 4. Chromatographic separations on succinic acid derivatized PS-DVB resin (Resin I) column $(50 \times 4.6 \mathrm{~mm})$ of $0.60 \mathrm{meq} / \mathrm{g}$ exchange capacity. Eluent conditions: $1.0 \mathrm{mM}$ ethylenediammonium, $0.30 \mathrm{mM}$ citrate (pH 5.4). Peaks are as identified. 


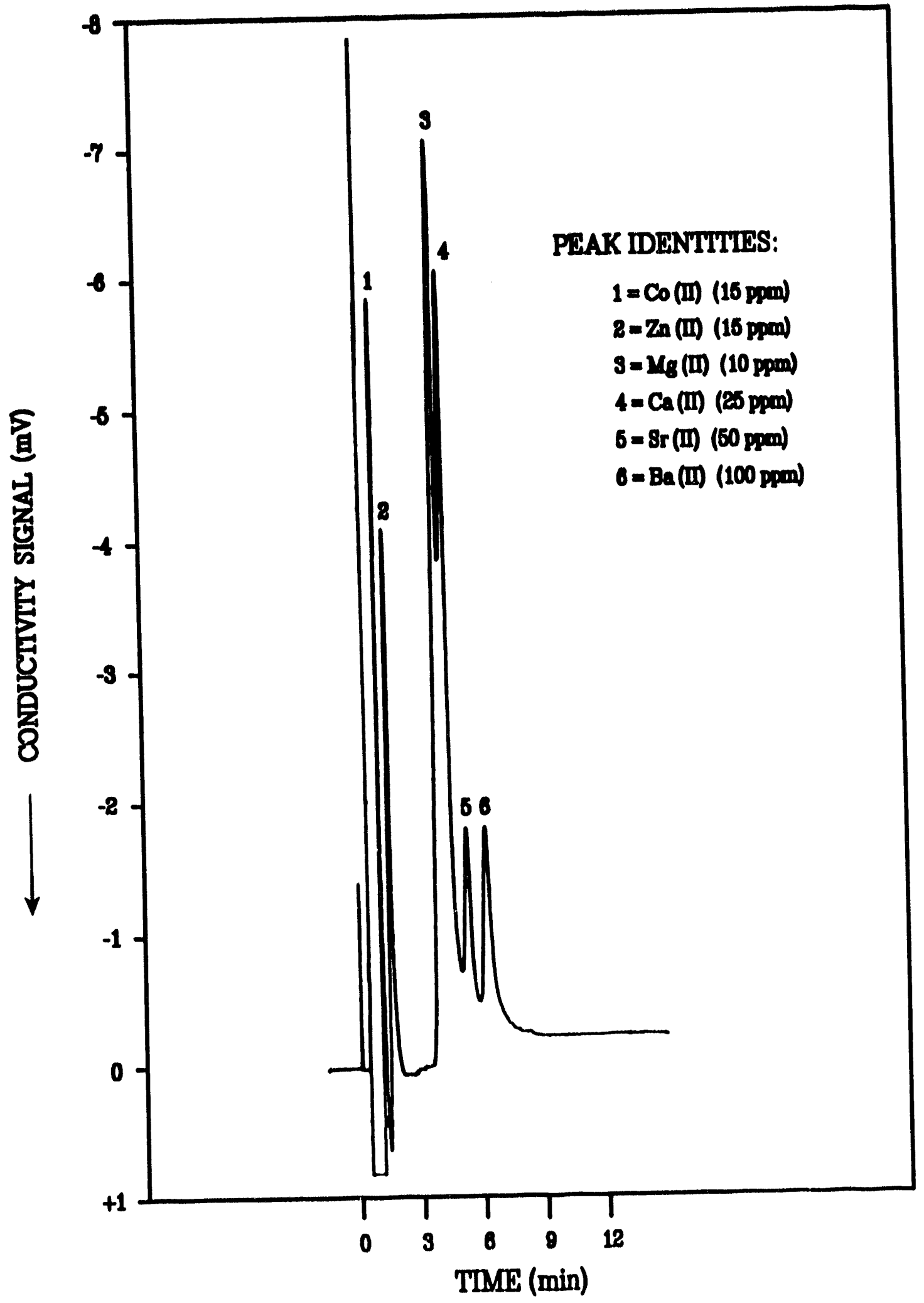




\section{Oxalate systems}

Preliminary experiments showed that good separations of several metal ions could be obtained with an eluent containing $1.0 \mathrm{mM}$ ethylenediammonium ion and 1.0 $m M$ oxalate. The separation of $\mathrm{Ni}^{++}\left(\right.$or $\left.\mathrm{Co}^{++}\right), \mathrm{Zn}^{++}, \mathrm{Mn}^{++}, \mathrm{Mg}^{++}, \mathrm{Ca}^{++}$, and $\mathrm{Ba}^{++}$was then optimized with respect to $\mathrm{pH}$. Virtually no separation was obtained at $\mathrm{pH} 4.0$ to 4.2 and the system peak was close to the metal ion peaks. By $\mathrm{pH} 4.4$ some separation had begun and the system peak was well removed from the vicinity of metal ion peaks.

The best separation occured around $\mathrm{pH} 5.3$ with all six peaks being well separated (Figure 5). Further increases in $\mathrm{pH}$ brought longer retention times, and by $\mathrm{pH} 5.8$ the quality of the separation had deteriorated noticeably. At pH 6.4 almost no separation was obtained.

The metal ion retention times were next reduced by increasing the oxalate concentration from $1.0 \mathrm{mM}$ to $1.6 \mathrm{mM}$. With this eluent, the optimum $\mathrm{pH}$ was approximately 5.8. It was possible to separate all seven metal ions in a mixture as shown in Figure 6.

The use of Resin $I I$ was next investigated. A short series of optimization experiments suggested the following conditions for separation: $0.75 \mathrm{mM}$ ethylenediammonium, $1.5 \mathrm{mM}$ oxalate and $\mathrm{pH} 4.5$ to 5.0. Figure 7 shows an excellent and rapid separation of $\mathrm{Zn}^{++}, \mathrm{Mn}^{++}, \mathrm{Mg}^{++}, \mathrm{Ca}^{++}$, and $\mathrm{Ba}^{++}$. Nickel (II) was partially separated from the $\mathrm{Zn}^{++}$peak. 
45

Figure 5. Chromatographic separations on Resin I column $(100 \times 4.6 \mathrm{~mm})$ of $0.39 \mathrm{meq} / \mathrm{g}$ exchange capacity. Eluent conditions: $1.0 \mathrm{mM}$ ethylenediammonium, $1.00 \mathrm{mM}$ oxalate $(\mathrm{pH} 5.3)$. Peaks are as identified. 


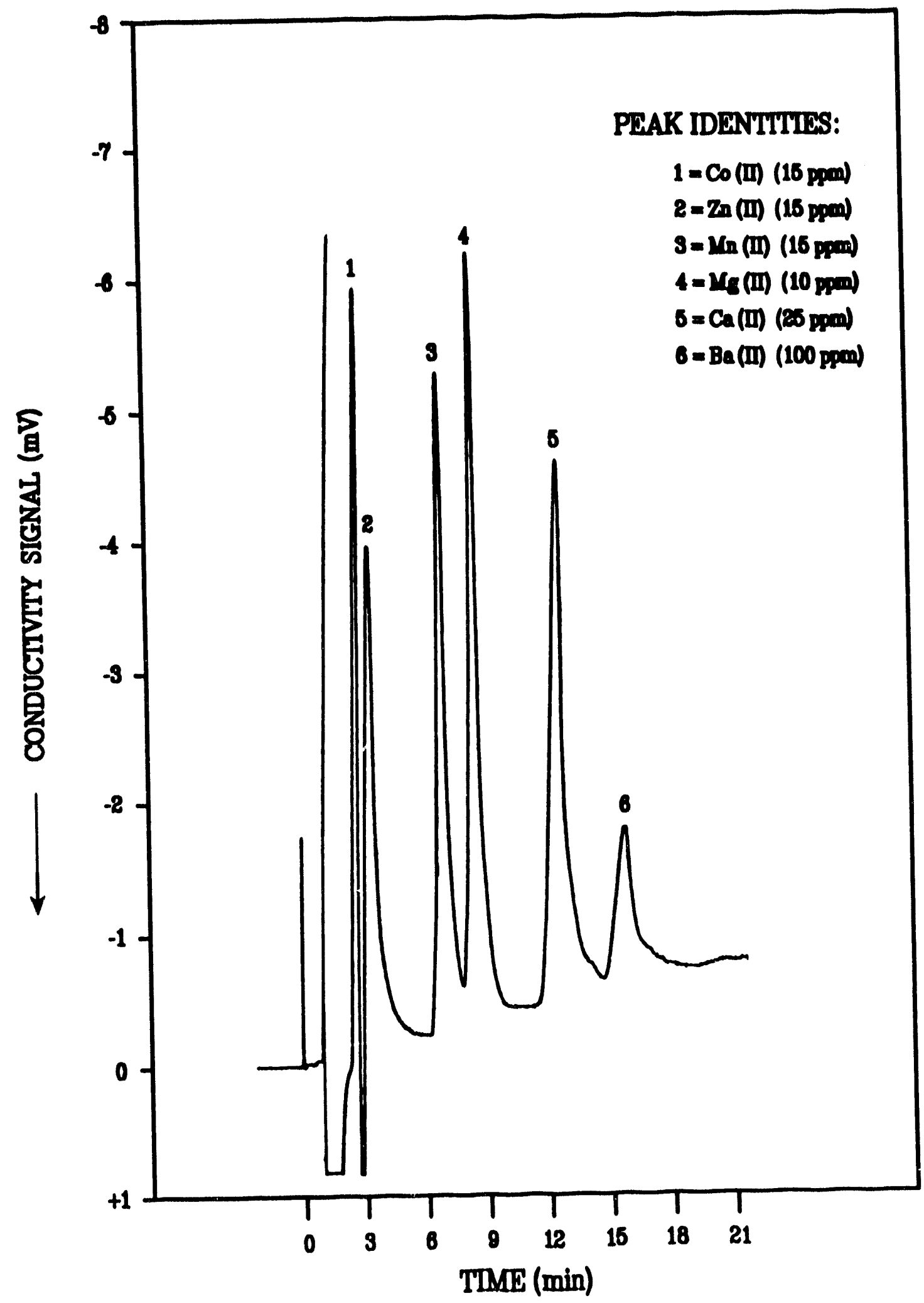




\section{7}

Figure 6. Chromatographic separations on Resin I column $(100 \times 4.6 \mathrm{~mm})$ of $0.60 \mathrm{meq} / \mathrm{g}$ exchange capacity. Eluent conditions: $1.0 \mathrm{mM}$ ethylenediammonium, $1.60 \mathrm{mM}$ oxalate $(\mathrm{pH} 5.8)$. Peaks are as identified. 


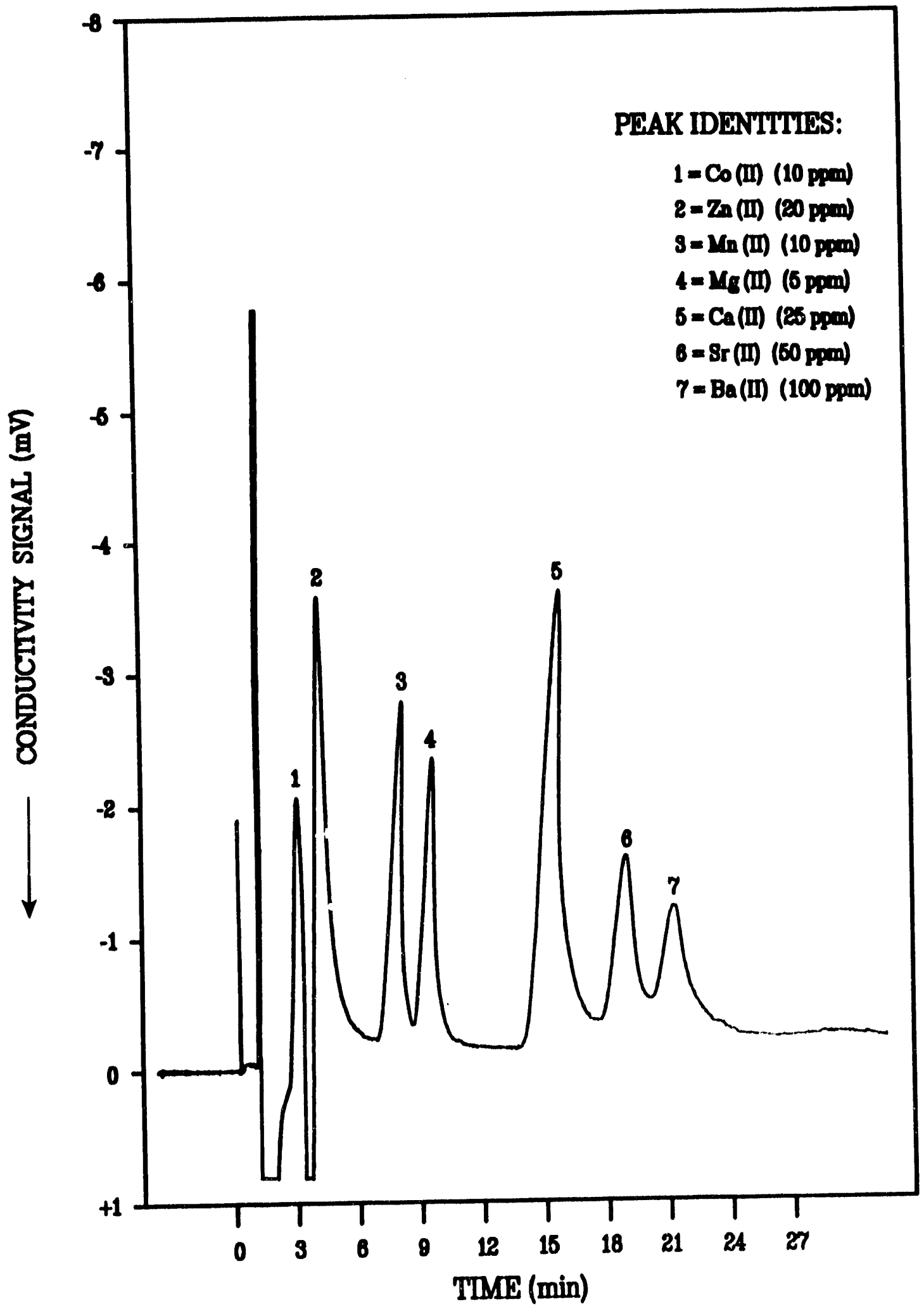




\section{9}

Figure 7. Chromatographic separations on benzoic acid derivatized PS-DVB resin (Resin II) column $(100 \times 4.6 \mathrm{~mm})$ of $0.39 \mathrm{meq} / \mathrm{g}$ exchange capacity. Eluent conditions: $0.75 \mathrm{mM}$ ethylenediammonium, $1.50 \mathrm{mM}$ oxalate $(\mathrm{pH} 4.5)$. Peaks are as identified. 


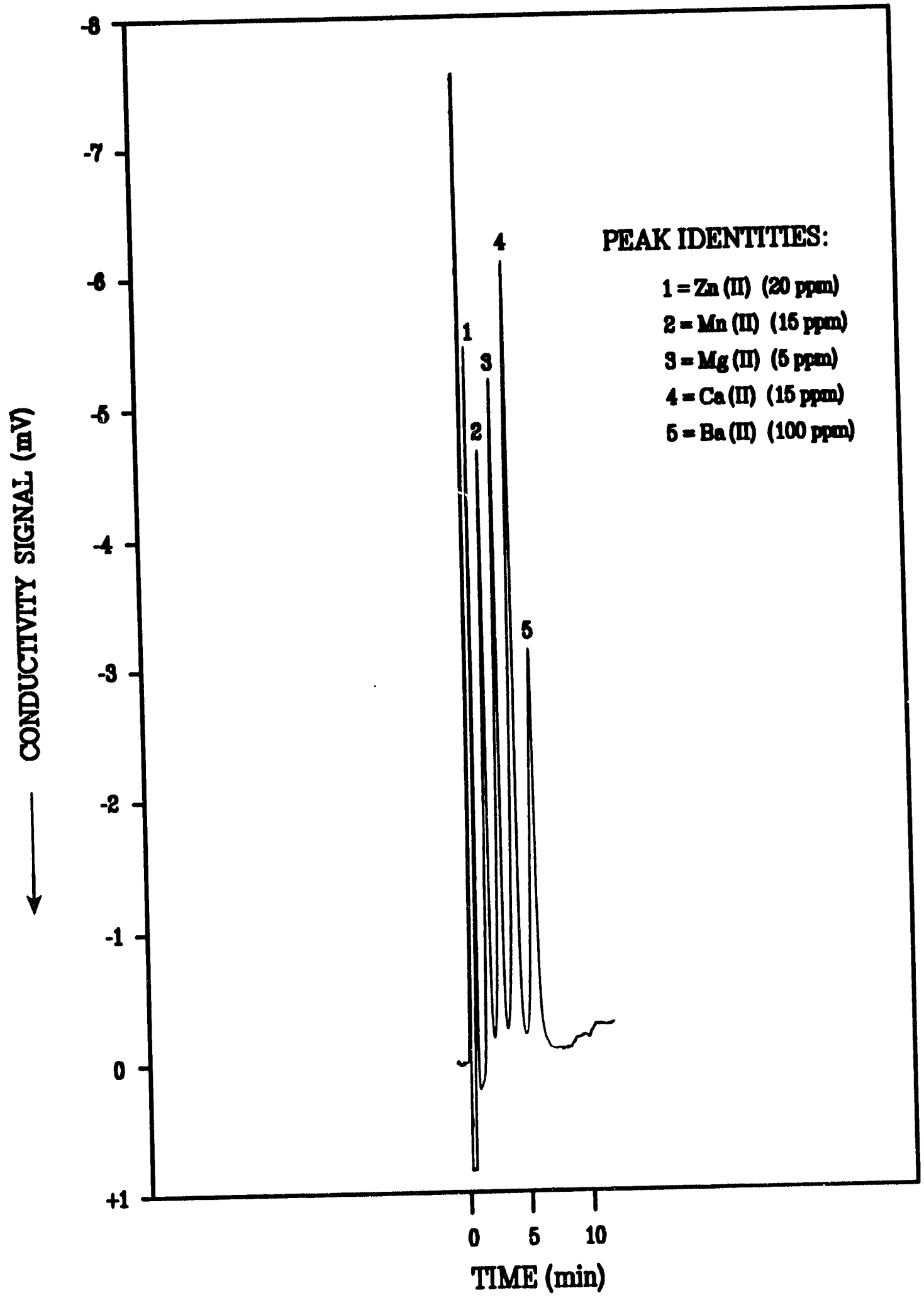


This separation (Figure 7) is similar to that obtained in Figure 6 using Resin $I$ except for the $\mathrm{pH}$ which is lower for the separations using Resin II. Based on the acid dissociation constants for the monomers of similar chemical structure, the acid strength of the carboxylic acid should be somewhat stronger for Resin II. Except for this, we could not discern any major differences in the two resins for chromatographic separations.

\section{Pyridine-2,6-dicarboxylic acid (PDA) systems}

PDA forms more stable complexes with most elements than oxalate or citrate. There is a reasonable difference in the formation constants of the PDA complexes listed in Table I. Preliminary optimization experiments showed that an excellent separation of magnesium (II) and the three alkaline earths could be obtained with an eluent containing $1.0 \mathrm{mM}$ ethylenediammonium and 0.05 or $0.1 \mathrm{mM}$ PDA, adjusted to pH 5.4 (see Figure 8). The order of elution is unusual in that magnesium(II) elutes after the alkaline earths. In other chromatographic systems, magnesium (II) elutes before calcium (II). Increasing the PDA concentration in steps from $0.025 \mathrm{mM}$ to 0.20 $m M$ decreases the retention times of $\mathrm{Ca}^{++}, \mathrm{Sr}^{++}$, and $\mathrm{Ba}^{++}$but has little effect on the retention time of $\mathrm{Mg}^{++}$. This effect was explained following theoretical considerations in earlier work by Sevenich and Fritz (1) in which a similar ethylenediammonium tartrate eluent was used. They derived a logarithmic equation relating adjusted retention time to the fraction of the metal ion in solution that exists as the free metal cation $\left(\alpha_{M}\right)$. The equation was tested for a number of cations and linear plots were obtained when the concentration of the complexing anion in the 


$$
52
$$

Figure 8. Chromatographic separations on Resin I column $(50 \times 4.6 \mathrm{~mm})$ of $0.60 \mathrm{meq} / \mathrm{g}$ exchange capacity. Eluent conditions: $1.0 \mathrm{mM}$ ethylenediammonium, $0.10 \mathrm{mM}$ PDA ( $\mathrm{pH}$ 5.4). Peaks are as identified. 


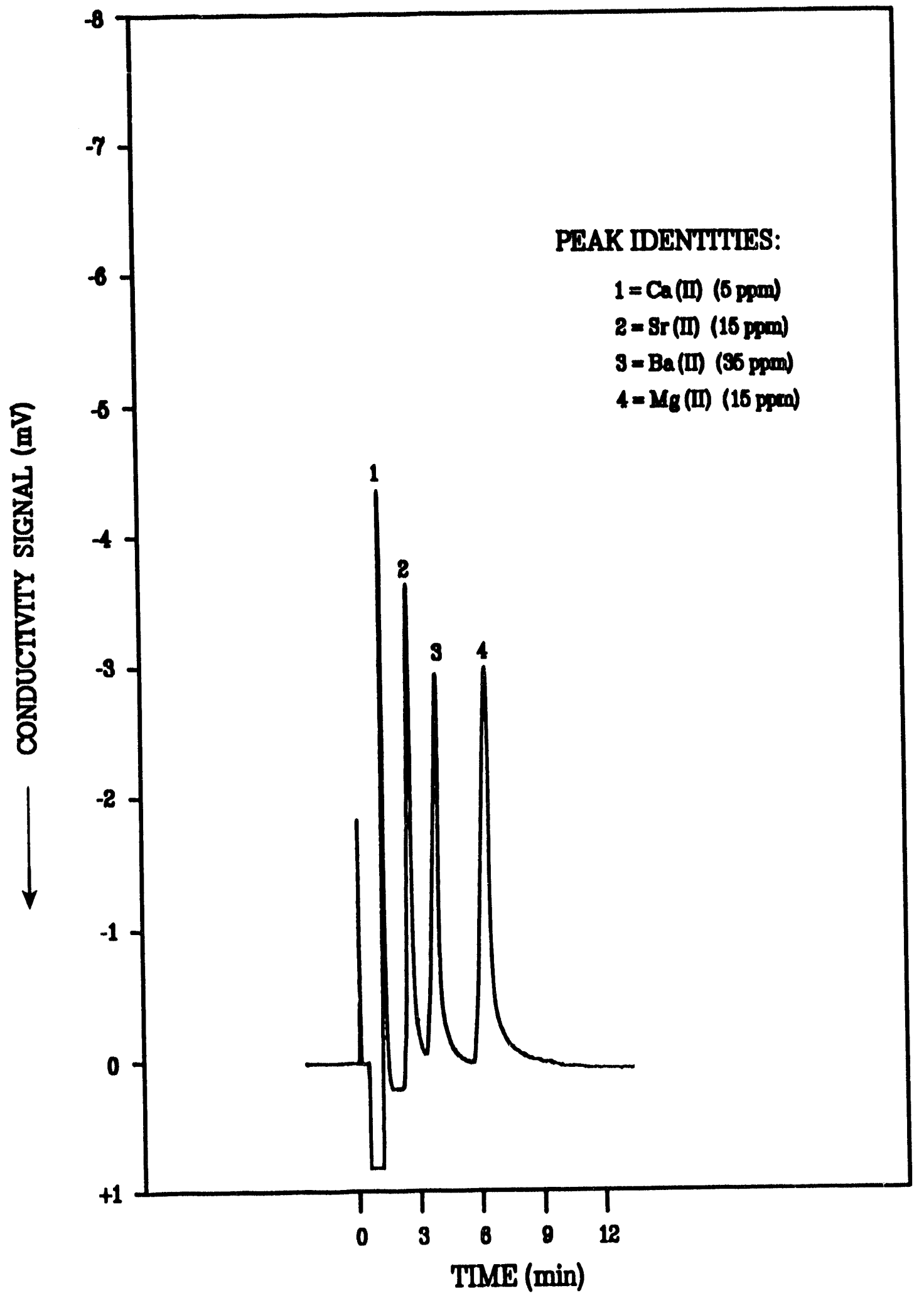


eluent was varied and $\log t_{R}^{\circ}$ was plotted against $\log \alpha_{M}$. In the present work using an ethylenediammonium / PDA eluent, when the concentration of PDA was increased from 0.025 to $0.200 \mathrm{mM}$, the following changes in $\alpha_{M}$ values were calculated: $\mathrm{Ca}^{++}(0.495$ to 0.095$) ; \mathrm{Sr}^{++}(0.863$ to 0.438$) ; \mathrm{Ba}^{++}(0.937$ to 0.649$) ; \mathrm{Mg}^{++}(0.995$ to 0.960). Linear plots were obtained for $\mathrm{Ca}^{++}, \mathrm{Sr}^{++}$, and $\mathrm{Ba}^{++}$when plotting $\log \mathrm{t}_{\mathrm{R}}$, against $\log \alpha_{M}$. In each case the value of $\alpha_{M}$ decreased by 30-40\% for the increased PDA concentration. Magnesium (II), however, showed only a 3.5\% decrease in $\alpha_{M}$ for the same increase in PDA concentration. This very weak complexation of $\mathrm{Mg}^{++}$by the PDA accounted for the negligible effect on the retention time of $\mathrm{Mg}^{++}$.

Using a longer column than that used to obtain Figure 8, the retention times of $\mathrm{Ca}^{++}, \mathrm{Sr}^{++}, \mathrm{Ba}^{++}$, and $\mathrm{Mg}^{++}$are somewhat increased allowing separation of some additional early-eluting ions. Figure 9 shows an excellent separation of $\mathrm{Zn}^{++}, \mathrm{Na}^{+}$, $\mathrm{Ca}^{++}, \mathrm{Sr}^{++}, \mathrm{Ba}^{++}$, and $\mathrm{Mg}^{++}$at $\mathrm{pH} 5.4$ on a $10-\mathrm{cm}$ column. Very similar chromatograms were obtained for samples in which $\mathrm{Co}^{++}$or $\mathrm{Cu}^{++}$was substituted for $\mathrm{Zn}^{++}$and $\mathrm{K}^{+}$or $\mathrm{NH}_{4}^{+}$was present instead of $\mathrm{Na}^{+}$. The same desired effect was achieved by increasing the $\mathrm{pH}$ from 5.4 to 5.8 using the shorter column. An equally good and somewhat faster separation was obtained at pH 5.8 on a $5 \mathrm{~cm}$-column.

Linear calibration curves were obtained for the ions separated by plotting peak area vs. concentration. The curves remained linear over the studied concentration range of $10^{-4}$ to $10^{-2} M$. 


$$
55
$$

Figure 9. Chromatographic separations on Resin I column $(100 \times 4.6 \mathrm{~mm})$ of $0.60 \mathrm{meq} / \mathrm{g}$ exchange capacity. Eluent conditions: $1.0 \mathrm{mM}$ ethylenediammonium, $0.05 \mathrm{mM}$ PDA (pH 5.4). Peaks are as identified. 


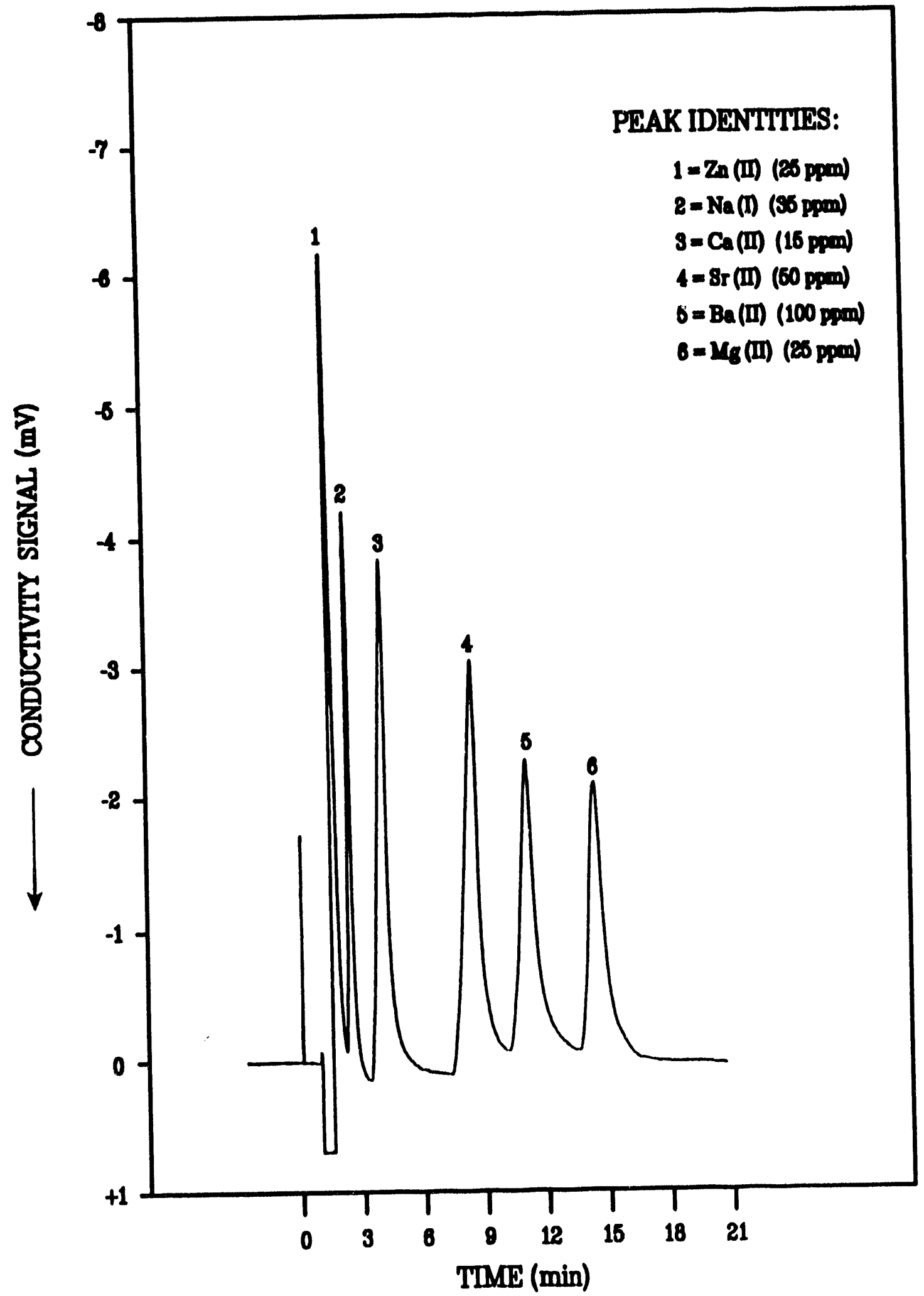




\section{CONCLUSIONS}

The desirable goal of separating an alkali metal from several divalent metal ions in a single run has thus been achieved. Unfortunately, we were not able to separate $\mathrm{Na}^{+}, \mathrm{K}^{+}$, and $\mathrm{NH}_{4}{ }^{+}$from one another. The sum of these monovalent ions can be obtained but this would be difficult to quantify because of the differing responses of the conductivity detector for these ions.

One final experiment was performed in which a PS-DVB resin was derivatized with multiple carboxylic acid groups. The idea was that the resin would have a higher number of available exchange sites to help separation of the monovalent cations. The resin performed in a similar fashion to those discussed with the additional capability of separating $\mathrm{Na}^{+}$and $\mathrm{NH}_{4}{ }^{+}$.

The presence of multiple weak-acid exchange sites was an improvement for the separation of monovalent cations but did not completely solve the problem. A final conclusion was made that a successful separation of the common monovalent cations $\left(\mathrm{Li}^{+}, \mathrm{Na}^{+}, \mathrm{K}^{+}\right.$, and $\left.\mathrm{NH}_{4}^{+}\right)$and the alkaline earth divalent cations $\left(\mathrm{Ca}^{++}, \mathrm{Mg}^{++}\right.$, $\mathrm{Sr}^{++}$, and $\mathrm{Ba}^{++}$) in a single run was not necessarily dependent upon the exchange capacity of the resin but on the strength of the acid functional group. The $\mathrm{pK}_{\mathrm{a}}$ 's of Resin $I$ and $I I$ discussed in the present work were approximately 5.6 and 4.2 respectively. If one were to explore the acid-strengthening effect of electronwithdrawing groups such as chloride, bromide, or hydroxide in the ortho position to the acid group, a cation exchange resin with a $\mathrm{pK}_{\mathrm{a}}$ value $<3$ could be realized. This 
would allow use of more acidic eluents without dropping below the $\mathrm{pK}_{\mathrm{a}}$ of the resin acid exchange group (which leads to loss of exchange capability). It is believed that this would permit separation of monovalent and divalent cations in a single run. 


\section{REFERENCES}

1. G.J. Sevenich and J.S. Fritz, Anal. Chem., 1983, 55, 12.

2. G.J. Sevenich and J.S. Fritz, J. Chromatogr., 1985, 347, 147.

3. H. Saitoh and K. Oikawa, J. Chromatogr., 1985, 329, 247.

4. H. Sato, Bunseki Kagaku, 1985, 34, 606.

5. B. Kondratjonok and G. Schwedt, Fres. Z. Anal. Chem., 1988, 332, 333.

6. D. Yan and G. Schwedt, Fres. Z. Anal. Chem., 1985, 320, 325.

7. S. Reiffenstuhl and G. Bonn, Fres. Z. Anal. Chem., 1988, 332, 130.

8. P. Kolla, J. Kohler and G. Schomburg, Chromatographia, 1987, 23, 465.

9. W. Coppock, J. Org. Chem., 1957, 22, 325. 
PAPER II. SEPARATION OF HYDROPHILIC ORGANIC ACIDS AND SMALL POLAR COMPOUNDS ON MACROPOROUS RESIN COLUMNS 


\section{SEPARATION OF HYDROPHILIC ORGANIC ACIDS AND SMALL POLAR COMPOUNDS ON MACROPOROUS RESIN COLUMNS}

John Morris and James S. Fritz

Ames Laboratory - U.S. Dept. of Energy and Department of Chemistry, Iowa State University Ames, IA 50011, U.S.A.

Reproduced with permission from $L C \cdot G C, 1993$, July, 11(7). Copyright 1993 Advanstar Communications. 


\section{SUMMARY}

Separation of carboxylic acids and other polar compounds by ion-exclusion chromatography is usually performed on a gel-type resin column of high cation exchange capacity (either sulfonated or carboxylated). It is now shown that separations can be obtained with lightly sulfonated macroporous resins packed into columns of smaller dimensions than have previously been reported. The predominant mechanism appears to be a partitioning between the primarily aqueous eluent and the resin matrix. Useful separations are also obtained on a low-capacity macroporous resin containing a carboxylic acid functional group. The use of organic solvents for controlling retention of organic acids with a more hydrophobic nature is explored. Aqueous-acetonitrile eluent gradients can be used to obtain fast separation of at least 11 different carboxylic acids in a single run. 


\section{INTRODUCTION}

Ion-exclusion chromatography (IEC) is a popular and practical way to separate weak inorganic and organic acids and other polar compounds of fairly low molecular weight. The separation is usually done on a sulfonated microporous cation-exchange column, although amines and ammonia have been separated on an anion-exchange column. When carboxylic acids are to be separated, a strong mineral acid is usually added to the eluent to repress ionization of the acids and ensure that they are in the molecular form.

Several reviews on IEC have been published (1-3). The separation mechanism has been described in several publications $(4,5)$. Molecular compounds are separated by differences in partitioning between the mobile phase (usually water) and the stagnant mobile phase inside the resin. Anions are excluded from entering the resin by a barrier of negatively charged sulfonate ions and pass rapidly through the column. The ability to separate carboxylic acids and other molecular substances from much larger quantities of ionic materials is one of the appealing advantages of ion-exclusion chromatography.

Ion-exclusion chromatography is usually carried out on gel-type resin columns with fairly large dimensions. The reason for this is that the solute retention volume $\left(V_{R}\right)$ is given by the equation: $V_{R}=V_{o}+K_{d} V_{i}$, where $V_{o}$ is the interstitial volume, $V_{i}$ is the volume of eluent within the resin beads, and $K_{d}$ is a distribution coefficient that can vary only from 0 to 1 . A large $V_{i}$ is needed for good separations because of 
this narrow range of $\mathbf{K}_{\mathbf{d}}$.

It is recognized that partitiuning can also occur based on hydrophobic attraction of the sample solutes to the polymeric resin matrix $(6,7)$. A low percentage of methanol or acetonitrile is often added to the aqueous eluent to reduce or eliminate this hydrophobic interaction. One commercial column (HPICE AS-5, Dionex Corp.) contains both sulfonic acid and carboxylic acid functional groups (1). It is claimed that this column operates on the mechanism of ion exclusion, hydrophobic interactions, and hydrogen bonding (8).

Gjerde and Mehra (1) give tables of capacity factors $\left(k^{\prime}\right)$ for a number of organic acids on several commercial columns that are widely used for IEC. Examination of these results yields some interesting observations. If the mechanism for separation of these carboxylic acids is primarily one of ion exclusion, why should an aliphatic carboxylic acid with a larger $\mathbf{R}$ group elute later than one with a smaller $R$ group? If anything, the $K_{d}$ value of the more bulky acid might be expected to be lower than that of the less bulky acid. Again, why should substitution of an aliphatic-H by an -OH group make the hydroxyl compound elute faster? (For example, the elution order is tartaric < malic < succinic).

Interaction of the solute acid with the resin matrix seems a more reasonable explanation. An acid with a larger $\mathbf{R}$ group would have a greater hydrophobic attraction and thus a longer retention time. A hydroxy compound is more polar than its hydrogen analogue and thus would interact less strongly with the resin matrix. 
In IEC stronger acids (lower $\mathrm{pK}_{\mathrm{a}}$ ) are known to elute more rapidly than weaker acids. Presumably this is because the stronger acids are incompletely converted to the molecular form. However, if this were the case, co-existence of the ionic and molecular forms might lead to broader peaks. Actually, these compounds have very sharp peaks. A better explanation seems to be that stronger acids are more polar and therefore interact less strongly with the resin matrix.

These considerations reinforce the idea that the predominant mechanism for chromatographic separation of carboxylic acids is differences in partitioning between the mobile and resin phases (i.e. hydrophobic attraction) and that an ion-exclusion mechanism is not essential. It should be possible to obtain good separations on macroporous resin columns that have significantly smaller diameters and lengths than the microporous (gel) resin columns that are now used. This could lead to faster separations. These ideas are examined in the experimental part of the present paper, and it is shown that excellent separations of low-molecular weight organic acids can indeed be obtained on relatively small columns packed with macroporous resins. 


\section{EXPERIMENTAL}

\section{Apparatus}

The chromatographic system consisted of an LKB 2156 solvent conditioner, an LKB 2249 HPLC gradient pump (LKB, Bromma, Sweden) with a mixing chamber volume of $0.5 \mathrm{ml}$, a Rheodyne 7125 injector (Rheodyne, Berkeley, CA) equipped with a $20-\mu 1$ loop, a Kratos Spectroflow 783 UV-Vis detector (Applied Biosystems, Ramsey, NJ), a ERMA ERC-7510 refractive index detector (ERMA Optical Works, Tokyo, Japan), and a Fisher Recordall series 5000 recorder (Fisher Scientific/Instrument Lab, Itasca, IL). The data acquisition system consisted of a Keithley Chrom 1-AT acquisition board (Keithley MetraByte Corp., Taunton, MA) and Labtech Notebook software (Laboratory Technologies Corp., Wilmington, MA). The ion-exclusion columns used in this study were a Hamilton PRP-X300 (150 mm x $4.6 \mathrm{~mm}$ ID) column containing macroporous 7- $\mu \mathrm{m}$ polystyrene-divinylbenzene resin sulfonated to an exchange capacity of 0.17 meq/g (Hamilton Co., Reno, NV), a Tosoh TSK-GEL (300 mm x 7.8 mm ID) column containing microporous $5-\mu \mathrm{m}$ polyacrylate resin carboxylated to an exchange capacity of $4.2 \mathrm{meq} / \mathrm{g}$ (Tosoh Corp., Japan), and columns prepared in our lab using 5 or $8-\mu \mathrm{m}$ macroporous polystyrene-divinylbenzene resins (Sarasep Inc., Santa Clara, CA) functionalized with either a carboxylic acid group or a sulfonic acid group. A Shandon HPLC packing pump (Shandon Southern, Sewickley, PA) was used for column packing. 


\section{Reagents and chemicals}

The organic solvents used for the chromatographic separations were HPLC grade. Stock solutions of the organic acids were prepared from reagent grade materials. Eluents were prepared daily using reagent grade sulfuric acid. A Barnstead Nanopure II system (Sybron Barnstead, Boston, MA) was used to further deionize distilled water for all eluents and sample mixtures.

\section{Synthesis procedures and capacity determination}

The synthesis of the carboxylic acid derivatized resin was described previously. The sulfonic acid derivative was prepared by reacting the resin with concentrated sulfuric acid. Approximately 2 grams of dry resin was placed in a beaker and slurried with $10 \mathrm{ml}$ glacial acetic acid while stirring. Concentrated sulfuric acid $(25 \mathrm{ml})$ was added and stirred for 2 mins. at room temperature. The reaction was then quenched by pouring the reaction mixture into a ice/methanol mixture. The resin was collected by filtration, washed thoroughly with methanol and water, and dried. The capacity was determined by acid-base titration to be $0.4 \mathrm{meq} / \mathrm{g}$.

The sulfonation reaction proceeded very rapidly as a color change from white to purple was noticed instantaneously as the sulfuric acid was added. The bezene rings on the outer surface undergo reaction very quickly. Longer reaction times are required to obtain higher capacities as the sulfuric acid needs time to penetrate into the inner pores of the resin particle. 


\section{RESULTS AND DISCUSSION}

Lee and others (9-12) have obtained good separations of carboxylic acids on a macroporous resin column with only a low amount of sulfonation $(0.17 \mathrm{meq} / \mathrm{g})$. Using a self-prepared column containing macroporous 5- $\mu \mathrm{m}$ PS-DVB resin sulfonated to an exchange capacity of $0.25 \mathrm{meq} / \mathrm{g}$, we were able to confirm this as demonstrated by the separation of nine carboxylic acids in Figure 1 using an aqueous eluent containing 1 $m M$ sulfuric acid. However, the peaks for glutaric and propionic acid were broad and butyric acid failed to elute. Using the same column and an eluent containing 5\% acetonitrile, instead of water alone, a good separation was obtained for formic, acetic, propionic, and butyric acids (Figure 2).

For comparison, several aliphatic carboxylic acids were separated on a highly carboxylated polyacrylate gel column of the type commonly used for ion-exclusion separations. Figure 3 shows a separation of the acids separated in Figure 2 using an entirely aqueous eluent. Apparently the hydrophobic attraction of propionic and butyric acids for the polymeric resin is much less on the gel resin than it is on the lightly sulfonated macroporous resin. The first peak to elute (formic acid) has a much longer retention time on the gel resin owing to the larger amount of the gel resin that was used.

The next column examined was packed with a macroporous polymeric resin bearing carboxylic acid groups at an exchange capacity of $0.39 \mathrm{meq} / \mathrm{g}$. Preparation of these resins has been described (13). As before, the eluent contained $1 \mathrm{mM}$ sulfuric 
68

Figure 1. Isocratic separation on sulfonated PS/DVB resin column (150 $\mathrm{x}$ $4.6 \mathrm{~mm}$ I.D.) of $0.25 \mathrm{meq} / \mathrm{g}$ exchange capacity. Eluent conditions: $1.0 \mathrm{mM}$ sulfuric acid ( $\mathrm{pH} 2.7$ ). Detection: UV absorbance at 210 nm. Flow rate: $2.0 \mathrm{ml} / \mathrm{min}$. Peaks are as identified. 


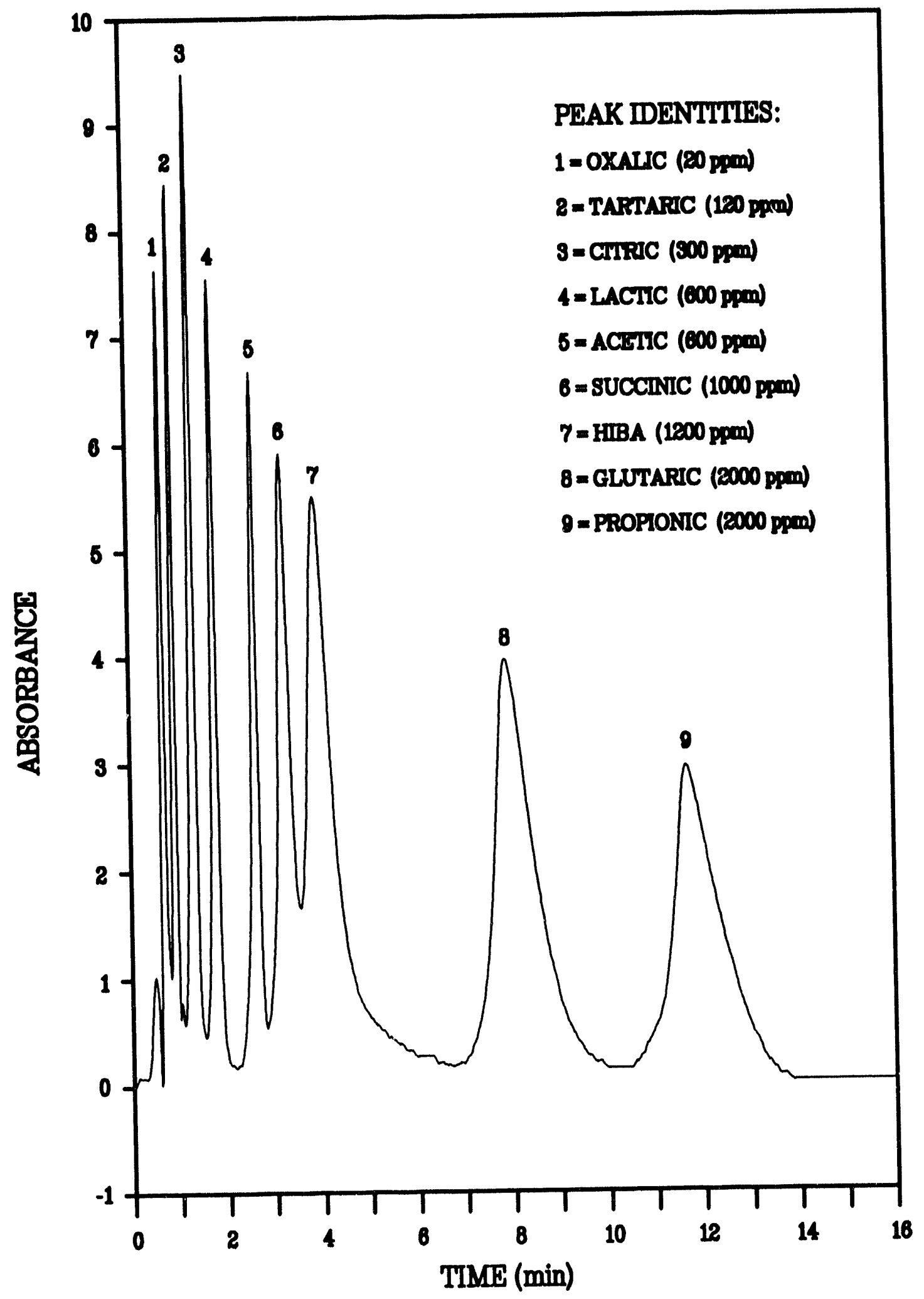


Figure 2. Isocratic separation on sulfonated PS/DVB resin column $(150 \mathrm{x}$ $4.6 \mathrm{~mm}$ I.D.) of $0.25 \mathrm{meq} / \mathrm{g}$ exchange capacity. Eluent conditions: $1.0 \mathrm{mM}$ sulfuric acid (pH 2.7), 5\% acetonitrile. Detection: UV absorbance at $210 \mathrm{~nm}$. Flow rate: $2.0 \mathrm{ml} / \mathrm{min}$. Peaks are as identified. 


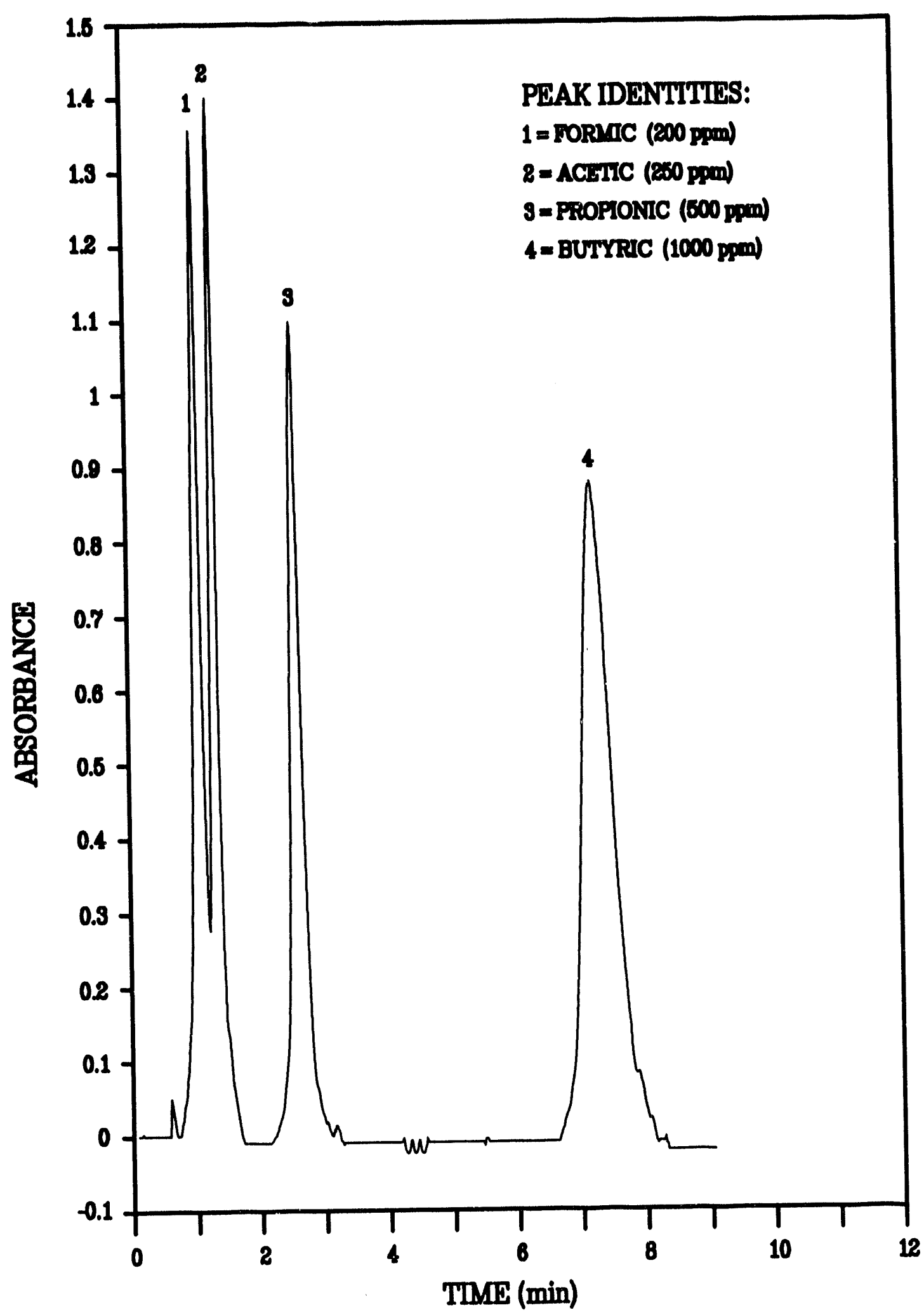

FIGURE 2 
Figure 3. Isocratic separation on TOSOH TSK-GEL SCX IEC column (300 x $7.8 \mathrm{~mm}$ I.D.) of $4.2 \mathrm{meq} / \mathrm{g}$ exchange capacity. Eluent conditions: $1.0 \mathrm{mM}$ sulfuric acid ( $\mathrm{pH}$ 2.7). Detection: UV absorbance at 210 nm. Flow rate: $2.0 \mathrm{ml} / \mathrm{min}$. Peaks are as identified. 


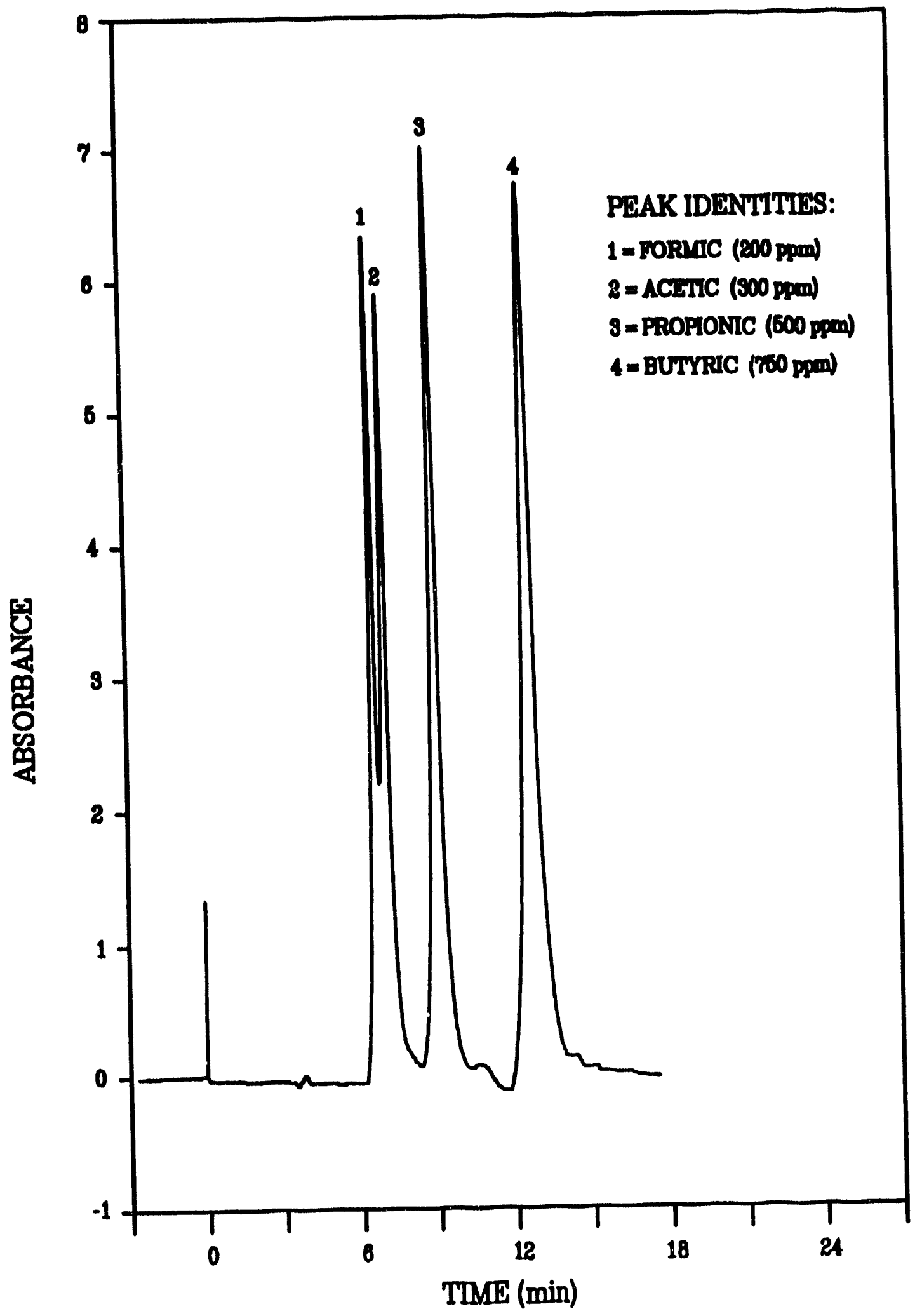


acid to repress the ionization of the alkyl carboxylic acids to be separated. This acidic eluent would also be expected to repress ionization of the resin carboxyl $\mathrm{gr}^{-\cdots} \mathrm{mr}$ Tin: would minimize electrostatic repulsion between the resin functional gro anionic form of the solute acids. This is unlike sulfonic acid resins where the sulfuriate anion persists even in the presence of an acidic eluent. Very good separations of polar carboxylic acids were obtained on the resin with the carboxyl group attached directly to the benzene ring of the polymer. The best separations were obtained with an acidic eluent containing $15 \%$ methanol to speed up elution of the later peaks (Figure 4).

A higher concentration of sulfuric ( $10 \mathrm{mM}$ instead of $1 \mathrm{mM}$ ) was added to the eluent in separations involving the benzoic acid resin. It was thought that this would convert a larger percentage of fairly strong carhnxylic acids to the molecular form and thereby make additional separations possi $\quad$ le effect in retention time or peak sharpness was noted for the stronger solute acids, but the later eluting acids showed longer retention times. Perhaps there is some salting-out effect taking place. At any rate, the concentration of sulfuric acid does affect the retention times of solute peaks. Table I shows the effect of eluent acid concentration on the retention for selected solute acids.

Chromatographic separation of carboxylic acids was tried on a more polar resin substituted with acetyl groups. This resin had been used with some success for HPLC separations (14). Some separation of hydrophilic carboxylic acids was obtained but the peaks were rather broad. This may have resulted from hydrogen bonding of the solutes with the resin acetyl group. 


\section{5}

Figure 4. Isocratic separation on benzoic acid-derivatized PS/DVB resin column (250 x $4.6 \mathrm{~mm}$ I.D.) of $0.39 \mathrm{meq} / \mathrm{g}$ exchange capacity. Eluent conditions: $1.0 \mathrm{mM}$ sulfuric acid ( $\mathrm{pH} 2.7$ ), $15 \%$ methanol. Detection: UV absorbance at $210 \mathrm{~nm}$. Flow rate: $2.0 \mathrm{ml} / \mathrm{min}$. Peaks are as identified. 


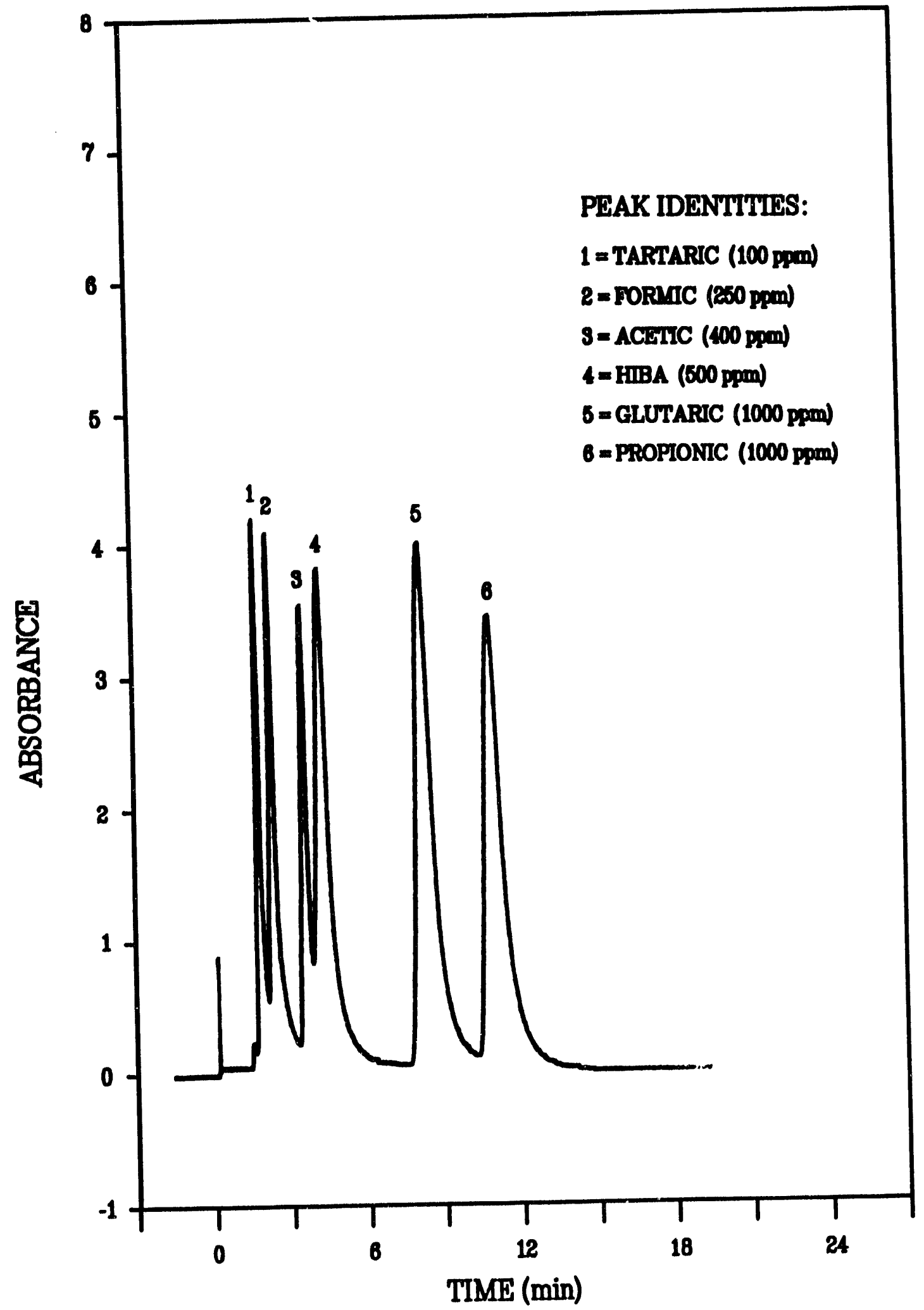


Table I. Retention times for various solute acids on benzoic acid resin column $(100 \times 4.6 \mathrm{~mm}$ I.D., $0.39 \mathrm{meq} / \mathrm{g}$ ) at two eluent sulfuric acid concentrations

\begin{tabular}{||l|c|c||}
\hline \multirow{2}{*}{ COMPOUND } & \multicolumn{2}{|c|}{$\mathrm{t}_{\mathrm{R}}(\mathrm{min})$} \\
\cline { 2 - 3 } & $1 \mathrm{mM} \mathrm{H}_{2} \mathrm{SO}_{4}$ & $10 \mathrm{mM} \mathrm{H}_{2} \mathrm{SO}_{4}$ \\
\hline \hline Tartaric & 0.90 & 0.85 \\
Malic & 1.05 & 1.10 \\
Formic & 1.05 & 1.20 \\
Malonic & 1.30 & 1.50 \\
Citric & 1.30 & 1.35 \\
Lactic & 1.45 & 1.70 \\
Acetic & 2.50 & 3.00 \\
Succinic & 2.90 & 3.70 \\
HIBA & 3.00 & 4.30 \\
Glutaric & 8.80 & 13.60 \\
Propionic & 14.20 & 18.20 \\
\hline
\end{tabular}


The next separations attempted were on a column packed with underivatized macroporous PS-DVB resin. Sharp peaks were obtained for several alkyl carboxylic acids and hydroxycarboxylic acids but the separations were not as good as on the carboxylic acid resin column. This stems from the much shorter retention times on the underivatized resin column. For example, the retention time of propionic acid is about one-half that on the carboxylic acid resin column with the same eluent. The carboxylic acid solutes would be expected to interact more strongly with a more polar resin than with the non-polar un-derivatized resin. Lee and Lord have reported shorter retention times for carboxylic acids on non-sulfonated resin columns than on sulfonated resins (9).

Unfunctionalized PS/DVB resin columns were tried with an eluent containing $\mathrm{Bu}_{4} \mathrm{~N}^{+} \mathrm{Cl}^{-}$or $\mathrm{OctSO}_{3}{ }^{-} \mathrm{Na}^{+}$. The idea was that the salt would equilibrate with the resin and form a hydrophilic coating. Little if any improvement was noted compared with the eluent containing no added salt. The $\mathrm{Bu}_{4} \mathrm{~N}^{+} \mathrm{Cl}^{-}$caused a bromide marker ion to elute later (with a broader peak). This was undoubtedly due to a dynamic ion-exchange mechanism. The $\mathrm{OctSO}_{3}{ }^{-\mathrm{Na}^{+}}$also showed little if any improvement. Increasing the concentration of this salt from $2 \mathrm{mM}$ to $10 \mathrm{mM}$ resulted in shorter retention times for the carboxylic acid solutes, especially for propionic and butyric acids. This could be a result of coating the resin by $\mathrm{OctSO}_{3}{ }^{-}$, thus reducing the surface area available to the solute carboxylic acids.

The previous experiments have supported the contention that carboxylic acids and hydroxycarboxylic acids can be separated effectively on macroporous resins that 
have a polar substituent such as a carboxylic acid or sulfonic acid functional group. An ion-exclusion mechanism, such as that postulated for gel-type resins, is not required. Rather fast separations should be possible, at least in some cases. Accordingly, use of a very short column with macroporous resin was investigated. Macroporous $5-\mu \mathrm{m}$ resin from Sarasep Inc. was sulfonated under mild conditions to give $0.4 \mathrm{meq} / \mathrm{g}$ exchange capacity. This was packed into a column only $5.0 \mathrm{~cm}$ long and used for separation of various carboxylic acids. Figure 5 shows an excellent separation of tartaric, formic, lactic, acetic, succinic and glutaric acids using an aqueous eluent. The first five acids are nicely separated in only about 2 min. This is significantly faster than any separation that has been reported with a conventional ion-exclusion column.

Compounds like glutaric acid and propionic acid elute more slowly with rather broad peaks using an aqueous eluent. An eluent containing a certain percentage of an organic solvent would reduce hydrophobic interaction of these acids with the resin and give sharper peaks. Lee, Bunker and Lord used a gradient of 6 to $54 \%$ methanol with some success, but they had to contend with a rising, non-linear baseline (9). We found that an effective gradient can be generated with a much lower percentage of acetonitrile. Figure 6 shows a separation of eight carboxylic acids in about 6 min. using aquecus eluent for $0.3 \mathrm{~min}$, followed by a water-acetonitrile gradient. After the gradient begins, the baseline begins to rise due to the absorbance by acetonitrile as illustrated in the inset chromatogram of Figure 6. The gradient was then run with no sample and the baseline determined. Using a data acquisition system both for the chromatographic and blank runs, it was possible to correct for the rising baseline. 
Figure 5. Isocratic separation on sulfonated PS/DVB resin column ( $50 \times 4.6$ $\mathrm{mm}$ I.D.) of $0.40 \mathrm{meq} / \mathrm{g}$ exchange capacity. Eluent conditions: 1.0 $m M$ sulfuric acid ( $\mathrm{pH} 2.7$ ). Detection: UV absorbance at $210 \mathrm{~nm}$. Flow rate: $2.0 \mathrm{ml} / \mathrm{min}$. Peaks are as identified. 


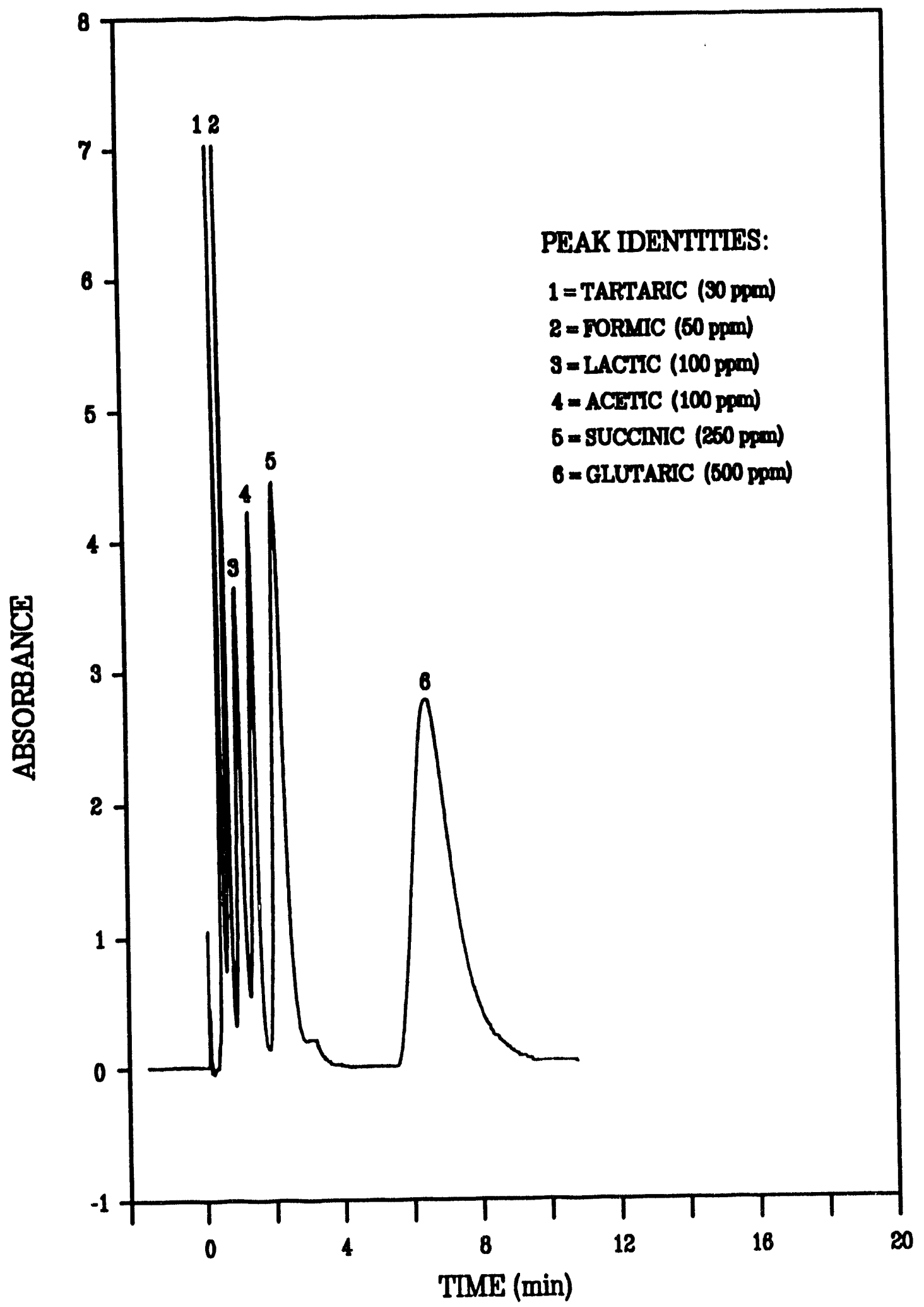




\section{2}

Figure 6. Gradient separation with background correction on sulfonated PS/DVB resin column (50 x $4.6 \mathrm{~mm}$ I.D.) of $0.40 \mathrm{meq} / \mathrm{g}$ exchange capacity. The inset shows the chromatogram before background subtraction. Eluent conditions: $1.0 \mathrm{mM}$ sulfuric acid ( $\mathrm{pH} 2.7)$ for 0.3 min., linear gradient from $0 \%$ to $20 \%$ acetonitrile over 4.2 min., then $2.5 \mathrm{~min}$. hold. Detection is UV absorbance at $210 \mathrm{~nm}$. Flow rate is $2.0 \mathrm{ml} / \mathrm{min}$. Peak identification: $1=\operatorname{tartaric}(250$ ppm), $2=$ formic $(400 \mathrm{ppm}), 3=$ lactic $(1000 \mathrm{ppm}), 4=$ acetic $(1000 \mathrm{ppm}), 5=\operatorname{succinic}(2000 \mathrm{ppm}), 6=$ propionic $(800 \mathrm{ppm})$, 7 = butyric $(1200 \mathrm{ppm}), 8=\operatorname{valeric}(2000 \mathrm{ppm})$. 


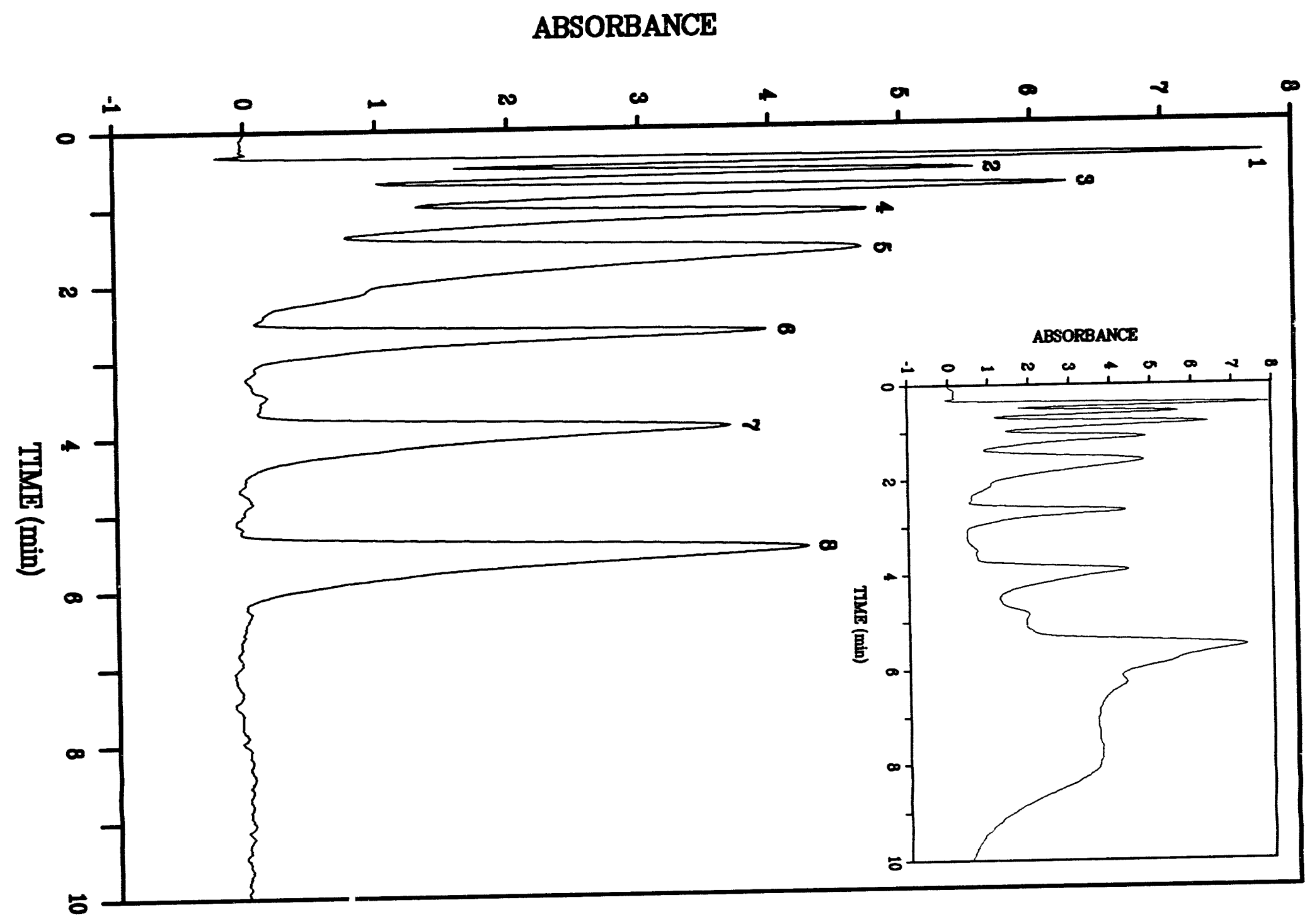


Still better separations can be obtained by using a longer column with gradient elution. Figure 7 shows an excellent separation of carboxylic acids obtained by subtracting the gradient background from the gradient chromatogram of the sample mixture. The separation was performed on the Hamilton PRP-X300 column. It should be noted that use of solvent gradients probably is not feasible with the gel resins commonly used for IEC since these resins are more prone to swelling or shrinking when the eluent composition is changed. The macroporous resin columns, however, are highly crosslinked, rigid, and can tolerate large differences in solvent polarity. Addition of organic solvents can be quite valuable in controlling selectivity, especially for more hydrophobic compounds.

Polar compounds other than carboxylic acids can also be separated on macroporous resin columns. Theses include alcohols, glycols, acetone, sugars, and weak inorganic acids. A refractive index detector is used. The chromatographic behavior of a number of polar compounds, including carboxylic acids, was compared on a lightly sulfonated resin of $0.25 \mathrm{meq} / \mathrm{g}$ exchange capacity and on the resin with a carboxylic acid functional group at an exchange capacity of $0.39 \mathrm{meq} / \mathrm{g}$. Capacity factors are given in Table II for isocratic elution with a strictly aqueous eluent and with eluents containing a low percentage of acetonitrile. In general, results on the two types of resin columns are not greatly different. In most cases, the retention times are similar or slightly larger on the carboxylic acid resin with an aqueous eluent. However, addition of a small percentage of acetonitrile seems to have a more pronounced effect on reducing the retention times on the carboxylic acid resin. 


\section{5}

Figure 7. Gradient separation with background correction on Hamilton PRP-X300 column ( $150 \times 4.1 \mathrm{~mm}$ I.D.) of $0.17 \mathrm{meq} / \mathrm{g}$ exchange capacity. Eluent conditions: $1.0 \mathrm{mM}$ sulfuric acid ( $\mathrm{pH} 2.7)$ for 1.0 min., linear gradient from $0 \%$ to $20 \%$ acetonitrile over $4.0 \mathrm{~min}$., then $5.0 \mathrm{~min}$. hold. Detection is UV absorbance at $210 \mathrm{~nm}$. Flow rate is $2.0 \mathrm{ml} / \mathrm{min}$. Peak identification: $1=$ oxalic $(20 \mathrm{ppm}), 2=$ tartaric $(120 \mathrm{ppm}), 3=$ malic $(360 \mathrm{ppm}), 4=\operatorname{citric}(300 \mathrm{ppm}), 5$ $=1$ actic $(600 \mathrm{ppm}), 6=\operatorname{acetic}(600 \mathrm{ppm}), 7=\operatorname{succinic}(350 \mathrm{ppm})$, $8=$ glutaric $(500 \mathrm{ppm}), 9=$ propionic $(500 \mathrm{ppm}), 10=$ butyric $(600 \mathrm{ppm}), 11=$ valeric $(1000 \mathrm{ppm})$. 


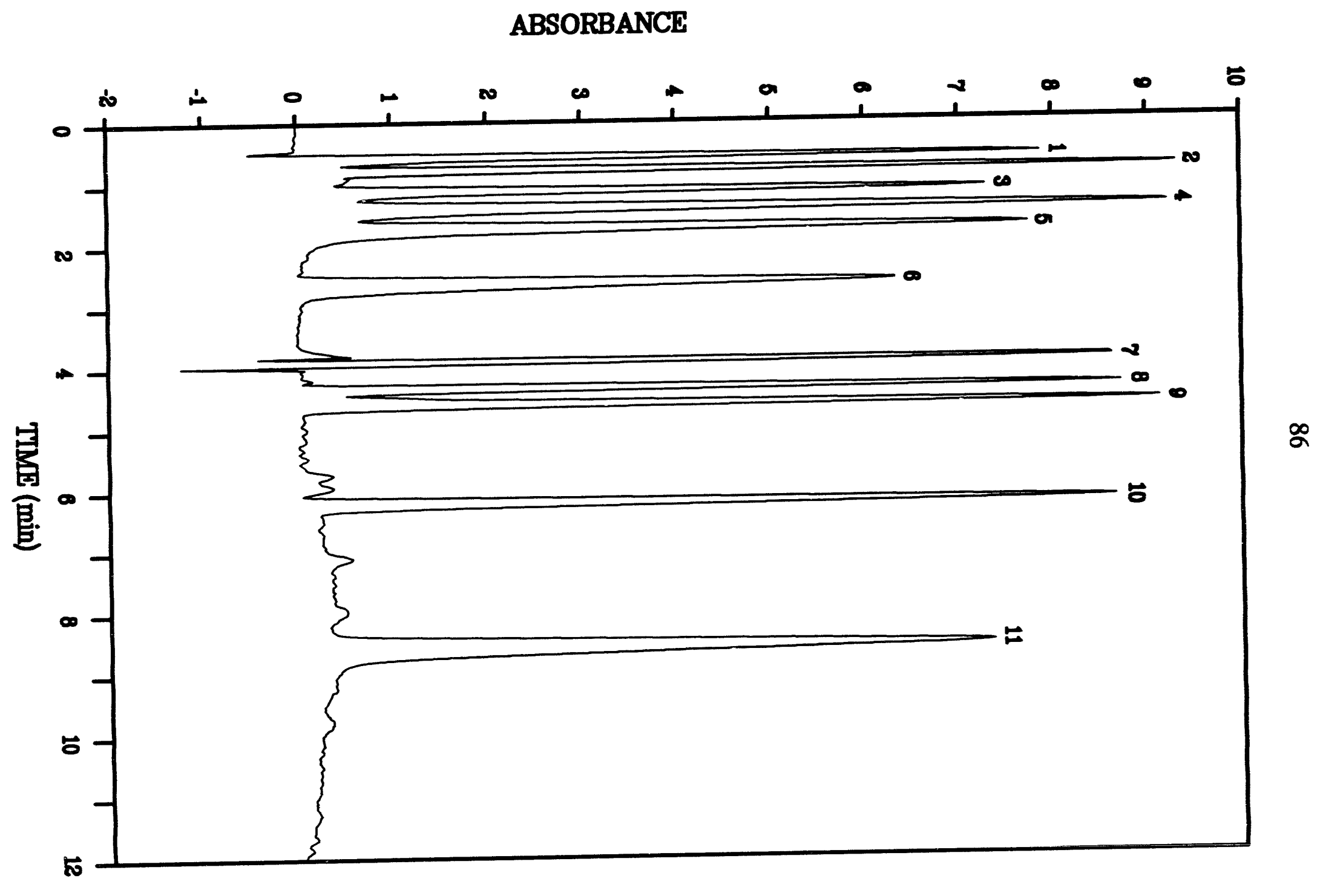


Table II. Capacity factors $\left(\mathrm{k}^{\prime}\right)$ for various compounds on carboxylic acid (0.39 $\mathrm{meq} / \mathrm{g})$ and sulfonated resin $(0.25 \mathrm{meq} / \mathrm{g})$ columns. Bromide was used as the marker for determining $t_{0}$

\begin{tabular}{|c|c|c|c|c|c|c|}
\hline \multirow{3}{*}{ COMPOUND } & \multicolumn{3}{|c|}{ RESIN- $\mathrm{CO}_{2} \mathrm{H}$} & \multicolumn{3}{|c|}{ RESIN-SO ${ }_{3} \mathrm{H}$} \\
\hline & \multirow[t]{2}{*}{$\mathrm{H}_{2} \mathrm{SO}_{4}(\mathrm{aq})$} & \multicolumn{2}{|c|}{$\mathrm{H}_{2} \mathrm{SO}_{4}+\mathrm{ACN}$} & \multirow[t]{2}{*}{$\mathrm{H}_{2} \mathrm{SO}_{4}(\mathrm{aq})$} & \multicolumn{2}{|c|}{$\mathrm{H}_{2} \mathrm{SO}_{4}+\mathrm{ACN}$} \\
\hline & & $2.5 \%$ & $5.0 \%$ & & $2.5 \%$ & $5.0 \%$ \\
\hline Formic & 0.69 & 0.40 & 0.33 & 1.52 & 0.72 & 0.67 \\
\hline Acetic & 3.61 & 1.08 & 0.73 & 2.92 & 1.41 & 0.75 \\
\hline Propionic & 23.04 & 4.42 & 2.00 & 14.96 & 4.52 & 3.00 \\
\hline Butyric & --- & 20.44 & 9.45 & $-\cdots$ & 17.79 & 9.00 \\
\hline Iso-Butyric & $\cdots$ & $\cdots$ & 9.39 & $\cdots$ & --- & 8.58 \\
\hline Valeric & --- & $-\cdots$ & 39.30 & $\cdots$ & --- & 35.67 \\
\hline Oxalic & 0.25 & 0.25 & 0.23 & 0.06 & 0.06 & 0.06 \\
\hline Malonic & 1.12 & 0.65 & 0.45 & 1.06 & 0.68 & 0.66 \\
\hline Succinic & 4.23 & 1.12 & 0.64 & 3.38 & 1.52 & 0.75 \\
\hline Glutaric & 13.90 & 2.90 & 1.54 & 10.06 & 2.96 & 1.58 \\
\hline Tartaric & 0.33 & 0.17 & 0.15 & 0.42 & 0.42 & 0.42 \\
\hline Citric & 1.15 & 0.58 & 0.10 & 0.89 & 0.62 & 0.57 \\
\hline Lactic & 1.61 & 0.38 & 0.27 & 1.60 & 0.93 & 0.85 \\
\hline Malic & 0.81 & 0.35 & 0.27 & 0.83 & 0.67 & 0.59 \\
\hline HIBA & 5.15 & 1.23 & 0.82 & 3.42 & 1.76 & 1.33 \\
\hline Maleic & 3.85 & 1.62 & 1.50 & 1.40 & 0.36 & 0.31 \\
\hline Pyruvic & 1.32 & 0.44 & 0.44 & 0.94 & 0.43 & 0.39 \\
\hline Ascorbic & 0.83 & 0.44 & 0.31 & 1.12 & 0.87 & 0.74 \\
\hline Gluconic & 0.19 & 0.06 & 0.06 & 0.46 & 0.43 & 0.41 \\
\hline Methanol & 0.79 & 0.50 & 0.33 & 0.93 & 0.90 & 0.75 \\
\hline Ethanol & 3.15 & 0.96 & $\cdots$ & 2.47 & $\cdots$ & $-\cdots$ \\
\hline Propanol & 20.83 & 4.23 & 2.65 & 12.51 & 8.90 & 3.92 \\
\hline Butanol & $\cdots$ & $\cdots$ & 10.83 & -..- & 24.17 & 13.58 \\
\hline Ethylene glycol & 0.50 & 0.35 & 0.18 & 0.68 & 0.60 & 0.58 \\
\hline Glycerol & 0.35 & 0.19 & 0.16 & 0.53 & 0.47 & 0.25 \\
\hline Acetone & 25.92 & 4.23 & 2.73 & 12.42 & 4.30 & 3.08 \\
\hline $\mathrm{H}_{3} \mathrm{AsO}_{4}$ & 0.19 & 0.11 & 0.10 & 0.20 & 0.17 & 0.00 \\
\hline $\mathrm{HAsO}_{2}$ & 0.31 & 0.13 & 0.10 & 0.57 & 0.60 & 0.56 \\
\hline $\mathrm{H}_{2} \mathrm{CO}_{3}$ & 6.88 & 3.19 & 2.42 & 5.67 & $-\cdots$ & 3.61 \\
\hline $\mathrm{H}_{3} \mathrm{BO}_{3}$ & 0.29 & 0.15 & 0.16 & 0.61 & -.- & 0.19 \\
\hline
\end{tabular}




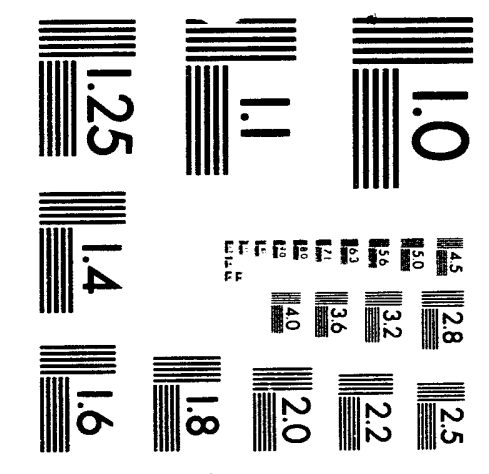



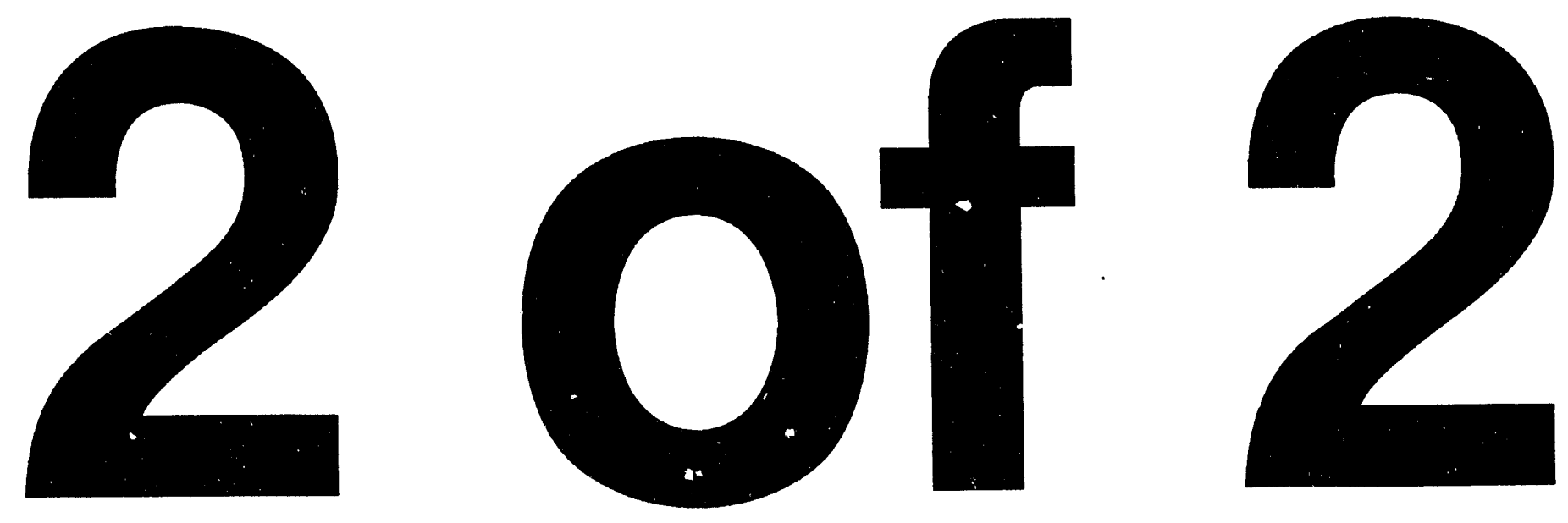
The Hamilton PRP-X300 column was used for the analysis of two wine samples with an aqueous eluent and refractive index detection (Figure 8). In this manner, it was possible to determine the major organic acid components as well as the amount of ethanol. 
Figure 8. Isocratic separations of wine samples after a 1:15 dilution on Hamilton PRP-X300 IEC column (150 x $4.1 \mathrm{~mm}$ I.D.) of 0.17 $\mathrm{meq} / \mathrm{g}$ exchange capacity. Eluent conditions: $1.0 \mathrm{mM}$ sulfuric acid (pH 2.7). Detection: refractive index. Flow rate: $1.0 \mathrm{ml} / \mathrm{min}$. Peak identification: 1 = unknown, 2 = unresolved sugars, tartaric, glycerol, $3=$ malic, $4=$ citric, $5=$ lactic, $6=$ ethanol, acetic, $7=$ succinic. 
SIGNAL

SIGNAL

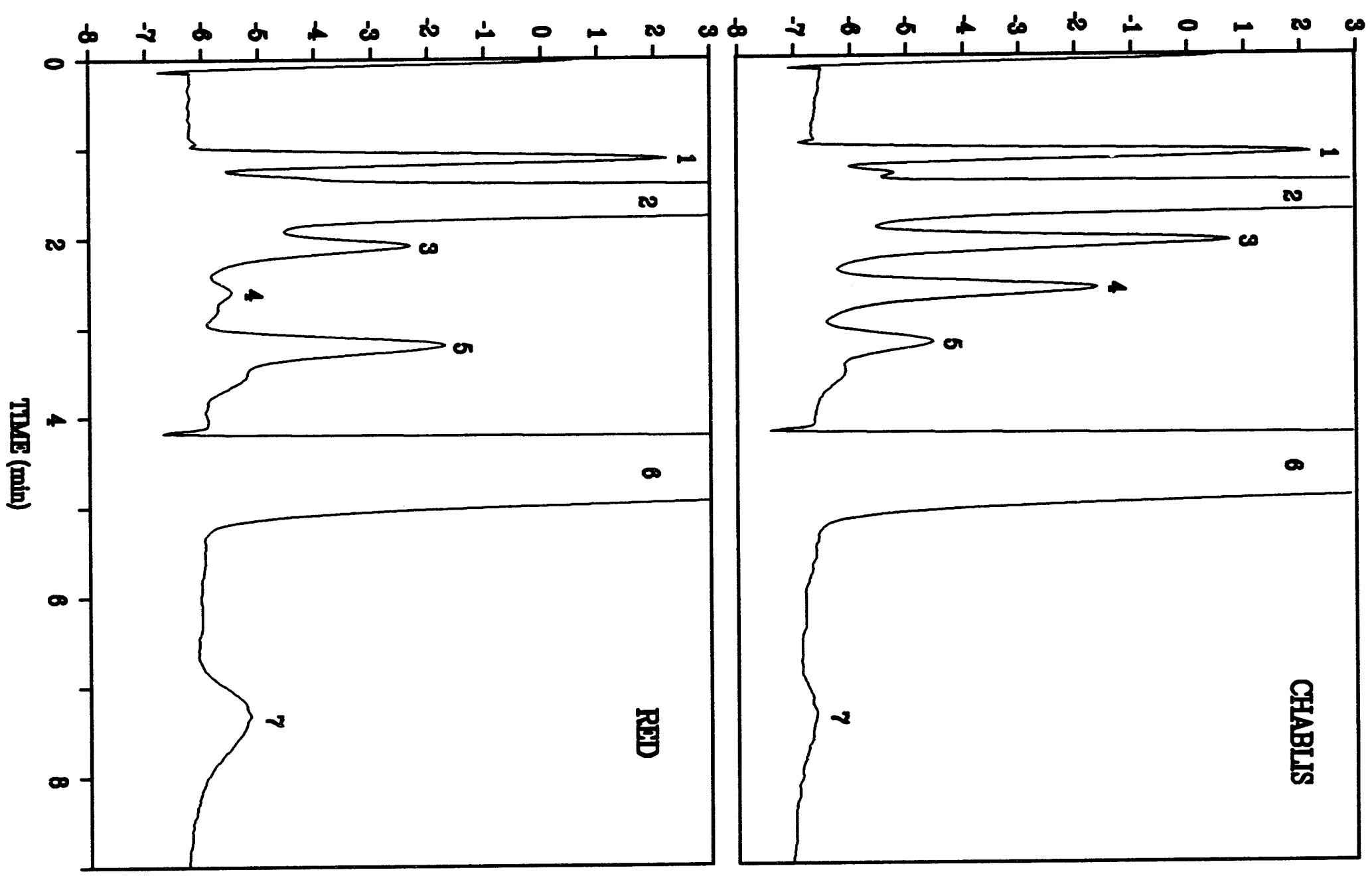




\section{CONCLUSIONS}

Excellent chromatographic separations of hydrophilic carboxylic acids are possible using an appropriately functionalized macroporous polymeric resin column. Since these resins are porous it is likely that they contain some stagnant mobile phase. However, the amount of stagnant phase would be much less than that of gel resin columns commonly used for IEC and not nearly enough to account for retention of any of the carboxylic acid solutes. The separation mechanism seems to be a partitioning of solutes between the mobile phase and the resin. An unfunctionalized polymeric resin can be used, but greater retention and better separations are obtained using a resin with polar substituents, such as sulfonate or carboxylate. Good separations were obtained on a carboxylic resin column under conditions where the resin carboxylic acid group would be mostly in the molecular rather than the ionized form.

Separations are significantly faster on an appropriate macroporous resin column than on the gel resin columns now used. It is possible to use a column as short as $\mathbf{5}$ $\mathrm{cm}$ with good results. Solvent gradients can be employed with macroporous resins to give faster separations and sharper peaks for later-eluting compounds. Correction of a rising baseline (using UV detection) is achieved with a data acquisition system. 


\section{REFERENCES}

1. D.T. Gjerde and H. Mehra, Advances in Ion Chromatography, Century International, 1989, Vol.1, 139.

2. P.R. Haddad and P.E. Jackson, Ion Chromatography, Elsevier, Amsterdam, 1990, 195.

3. J.S. Fritz, J. Chromatogr., 1991, 546, 111.

4. K. Tanaka, T. Ishizuka and J. Sunahara, J. Chromatogr., 1979, 174, 153.

5. B.K. Glód and W. Kemula, J. Chromatogr., 1986, 366, 39.

6. T. Jupille, M. Gray, B. Black, and M. Gould, Am. Lab., 1981, 13(8), 80.

7. D. Lee and A. Lord, $L C \cdot G C, 1987,5(3), 261$.

8. R.E. Smith, Applications of Ion Chromatography, CRC Press, Boca Raton, FL, 1988, 69.

9. D. Lee, M. Bunker and A. Lord, Advances in Ion Chromatography, Century International, 1989, Vol.1, 451.

10. P. Walser, J. Chromatogr., 1988, 439, 71.

11. D. Grosjean, A. Van Niste and S. Parmar, J. Liq. Chromatogr., 1989, 12, 3007.

12. D. Grosjean, Environ. Sci. Tech., 1991, 25, 710.

13. J.B. Morris and J.S. Fritz, J. Chromatogr., 1992, 602, 111.

14. J. Sun and J.S. Fritz, J. Chromatogr., 1990, 522, 95. 
PAPER III. USE OF ELUENT MODIFIERS FOR THE LIQUID CHROMATOGRAPHIC SEPARATION OF CARBOXYLIC ACIDS USING CONDUCTIVITY DETECTION 
USE OF ELUENT MODIFIERS FOR THE LIQUID

CHROMATOGRAPHIC SEPARATION OF CARBOXYLIC ACIDS USING CONDUCTIVITY DETECTION

John Morris and James S. Fritz

Ames Laboratory - U.S. Dept. of Energy and

Department of Chemistry, Iowa State University

Ames, IA 50011, U.S.A.

Submitted July, 1993 


\section{SUMMARY}

Small straight-chain alcohols added to an aqueous mobile phase have a dramatic effect on the chromatographic behavior of alkane carboxylic acids. The alcohols were believed to coat the resin surface and establish a dynamic equilibrium between the mobile and stationary phases. This altered the hydrophilicity of the resin surface permitting a fine-tuning of the stationary phase capacity behavior. The eluent systems have a very low background conductance, thus allowing very sensitive conductometric detection. Carbonic acid (from atmospheric carbon dioxide) in the eluent was determined to play a role in the retention mechanism of the weak-acid species. The use of larger straight-chain alcohols as modifiers in a water/acetonitrile mobile phase was investigated for the separation of neutral organic species. Finally, a 12-carbon alcohol was semi-permanently coated on the PS-DVB resin. This treated column was used for separation of alkane carboxylic acids using a purely aqueous eluent and conductometric detection. 


\section{INTRODUCTION}

Weak inorganic and organic acids of fairly low molecular weight are often separated and determined by ion-exclusion chromatography (IEC). Such separations are usually performed on sulfonated macroporous (gel) resin columns of fairly large dimensions. Several reviews on IEC have been published (1-3). The separation mechanism is considered to be the partitioning of solute acids between the predominately aqueous eluent and water inside the resin gel, although hydrophobic attraction is also known to play a role $(4,5)$.

Hydrophilic organic acids can also be separated on smaller resin columns containing lightly sulfonated macroporous (non-gel) polymeric resins $(6,7)$. Following accepted theory, these separations were performed with an eluent containing sulfuric acid. The purpose of the acid is to repress ionization of the carboxylic acids being separated so that the mechanism of separation would be predominantly interaction of the molecular solute acid with the resin matrix (i.e. hydrophobic attraction). Separations using macroporous resins offered some real advantages over traditional separations performed on gel resins. Separations were much faster (on much smaller columns), and the rigid nature of these resins provided the additional ability to carry out gradient elution with an organic solvent such as acetonitrile.

Detection of the separated carboxylic acids was by UV absorbance (at $210 \mathrm{~nm}$ ) or refractive index. These methods are rather insensitive; typical sample concentrations were 20-2000 ppm. Unless an additional suppression device is used, the use of sulfuric 
acid in the eluent severely limits the use of conductivity detection because a small detection signal must be measured on top of a large background. Several methods have been used to overcome this situation. These methods fall under two basic strategies that were recently reviewed by Fritz (3). The first is to reduce the background conductance of the eluent by means of a suppressor column or membrane placed between the separation column and the detector cell. The second is to enhance the conductivity of the sample solutes through the use of a series of enhancement columns also placed after the separation column. Although these methods are effective, they do add to the complexity of the chromatographic system and introduce additional peak broadening. Perhaps a better alternative, when feasible, would be to develop a separation scheme that eliminates the need for these suppression or enhancement devices, while maintaining the effective use of conductivity detection.

Equilibrium constant calculations indicate that alkane carboxylic acids are extensively ionized (69-97\%) in predominantly aqueous solutions at the low concentrations generally used in liquid chromatography. It is questionable whether added sulfuric acid is really effective in converting the solute acids to the unionized molecular form. In fact Tanaka and coworkers (8) were able to obtain good separations of alkane carboxylic acids on gel-type resins with a carboxylic acid functional group using eluents containing a neutral hydrophilic chemical additive but no added acid.

In the present work, it was found that small straight-chain alcohols added to an aqueous eluent had a dramatic effect on the chromatographic behavior of small polar 
compounds such as the alkane carboxylic acids. The alcohols were believed to coat the surface of the polymeric resin owing to a dynamic equilibrium formed in which the alcohols were distributed between the mobile and stationary phases. In favorable cases, analytes at low ppb levels were easily detected by conductivity detection. This work was expanded to investigate the effect of larger straight-chain alcohols on a reversephase separation of neutral aromatic species where the eluent contained a fairly large percentage of organic solvent. Finally, a twelve-carbon alcohol was coated on a polymeric resin to form a semi-permanent coating. This was used for separation of alkane carboxylic acids with a strictly aqueous eluent. In separations involving the carboxylic acids, carbonic acid found naturally in the eluent had a noticeable effect on the retention mechanism of these weak-acid species. 


\section{EXPERIMENTAL}

\section{Apparatus}

The chromatographic system consisted of an LKB 2156 solvent conditioner, an LKB 2249 HPLC gradient pump (LKB, Bromma, Sweden) with a mixing chamber volume of $0.5 \mathrm{ml}$, a Rheodyne 7125 injector (Rheodyne, Berkeley, CA) equipped with a 20- $\mu \mathrm{l}$ loop, a Dionex CDM-3 conductivity detector (Dionex, Sunnyvale, CA), and a Fisher Recordall series 5000 recorder (Fisher Scientific/Instrument Lab, Itasca, IL). The data acquisition system consisted of a Keithley Chrom 1-AT acquisition board (Keithley MetraByte Corp., Taunton, MA) and Labtech Notebook software (Laboratory Technologies Corp., Wilmington, MA). The ion-exclusion columns used in this study were prepared in our lab using $5-\mu \mathrm{m}$ underivatized macroporous polystyrenedivinylbenzene resin (Sarasep Inc., Santa Clara, CA) and the same resin functionalized with a sulfonic acid group at $0.25 \mathrm{meq} / \mathrm{g}$ exchange capacity. A Shandon HPLC packing pump (Shandon Southern, Sewickley, PA) was used for column packing. A Hamilton PRP-X100 (Hamilton Co., Reno, NV) trimethylammonium anion-exchange column of $0.19 \mathrm{meq} / \mathrm{g}$ exchange capacity was used to remove carbonic acid from the eluent.

\section{Reagents and chemicals}

The organic eluent modifiers used for the chromatographic separations were reagent grade. Stock solutions of the organic acids were prepared from reagent grade materials. Eluents were prepared daily using the reagent grade organic modifiers and water. A Barnstead Nanopure II system (Sybron Barnstead, Boston, MA) was used to further deionize distilled water for all eluents and sample mixtures. 


\section{RESULTS AND DISCUSSION}

Experiments were designed to separate a mixture of alkane carboxylic acids using eluents containing no strong acid. In this manner, we could take advantage of the improved sensitivity of conductivity detection. We have experimented with a wide range of organic solvent/water eluent systems and found that the alkane carboxylic acids can be separated very nicely on the macroporous resins using conductivity detection.

The organic modifiers represent various functional groups. These include a series of straight-chain alcohols, cyclohexanol, dimethylformamide, tetrahydrofuran, methylethylketone, 2-ethoxyethanol, and acetonitrile. Some of these were effective in reducing the retention times of the carboxylic acid analytes and sharpening the peaks (see Table I). While all of the eluent modifiers had relative merits, the best appeared to be the straight-chain alcohols.

\section{Dynamic Coatings with Alcohol Modifiers}

\section{Carboxylic Acids}

Several carboxylic acids were separated chromatographically on a lightly sulfonated polymeric resin column. An aqueous eluent was used with varying amounts of various alcohols or other organic liquids as modifiers. Figure 1 shows the separation of 6 carboxylic acids using only water as an eluent. A couple of things should be noticed from this chromatogram. First, the elution order of the represented acids is different than in previous work using sulfuric acid in the eluent. In this case, succinic 
Table I. Retention times for formic, acetic, propionic, butyric, and valeric acids for optimized concentrations of various modifiers in binary water eluents. Separations performed on a lightly sulfonated resin column $(150 \mathrm{x}$ $4.6 \mathrm{~mm}$ ) of $0.25 \mathrm{meq} / \mathrm{g}$ exchange capacity. Detection was conductivity. Flow rate was $1.0 \mathrm{ml} / \mathrm{min}$.

\begin{tabular}{|c|c|c|c|c|c|}
\hline \multirow[b]{2}{*}{ Eluent Modifier } & \multicolumn{5}{|c|}{$t_{R}(\min )$} \\
\hline & Formic & Acetic & Propionic & Butyric & Valeric \\
\hline Water $(100 \%)$ & 1.35 & 3.90 & 17.90 & $\cdots$ & $\cdots$ \\
\hline Methanol (60\%) & 1.35 & 1.90 & 2.30 & 3.05 & 4.95 \\
\hline Ethanol $(40 \%)$ & 1.15 & 1.90 & 2.45 & 3.55 & 6.35 \\
\hline Propanol (20\%) & 0.90 & 1.45 & 1.95 & 2.55 & 4.65 \\
\hline Butanol (5\%) & 0.85 & 1.25 & 1.65 & 2.30 & 4.70 \\
\hline Pentanol (1\%) & 0.90 & 1.10 & 1.40 & 1.90 & 3.75 \\
\hline t-Butanol (5\%) & 0.80 & 1.10 & 1.85 & 3.55 & 8.50 \\
\hline Cyclohexanol (2\%) & 1.45 & 1.90 & 2.60 & 4.10 & $\cdots$ \\
\hline Dimethylformamide (15\%) & 1.35 & 2.05 & 3.70 & 8.40 & $\cdots$ \\
\hline Tetrahydrofurane (1\%) & 0.85 & 0.95 & 1.25 & 2.00 & 5.35 \\
\hline Methylethylketone (2\%) & 0.90 & 1.05 & 1.25 & 1.75 & 3.90 \\
\hline 2-Ethoxyethanol (10\%) & 0.95 & 1.20 & 1.70 & 2.80 & 7.85 \\
\hline Acetonitrile (20\%) & 0.90 & 1.35 & 1.75 & 2.40 & 4.40 \\
\hline
\end{tabular}




\section{1}

Figure 1. Chromatographic separation on sulfonated PS-DVB resin column $(150 \times 4.6 \mathrm{~mm})$ of $0.25 \mathrm{meq} / \mathrm{g}$ exchange capacity. Eluent conditions: deionized water. Detection is conductivity with an output range of $30 \mu \mathrm{S}$ full scale. Flow rate is $1.0 \mathrm{ml} / \mathrm{min}$. Peaks are as identified. 


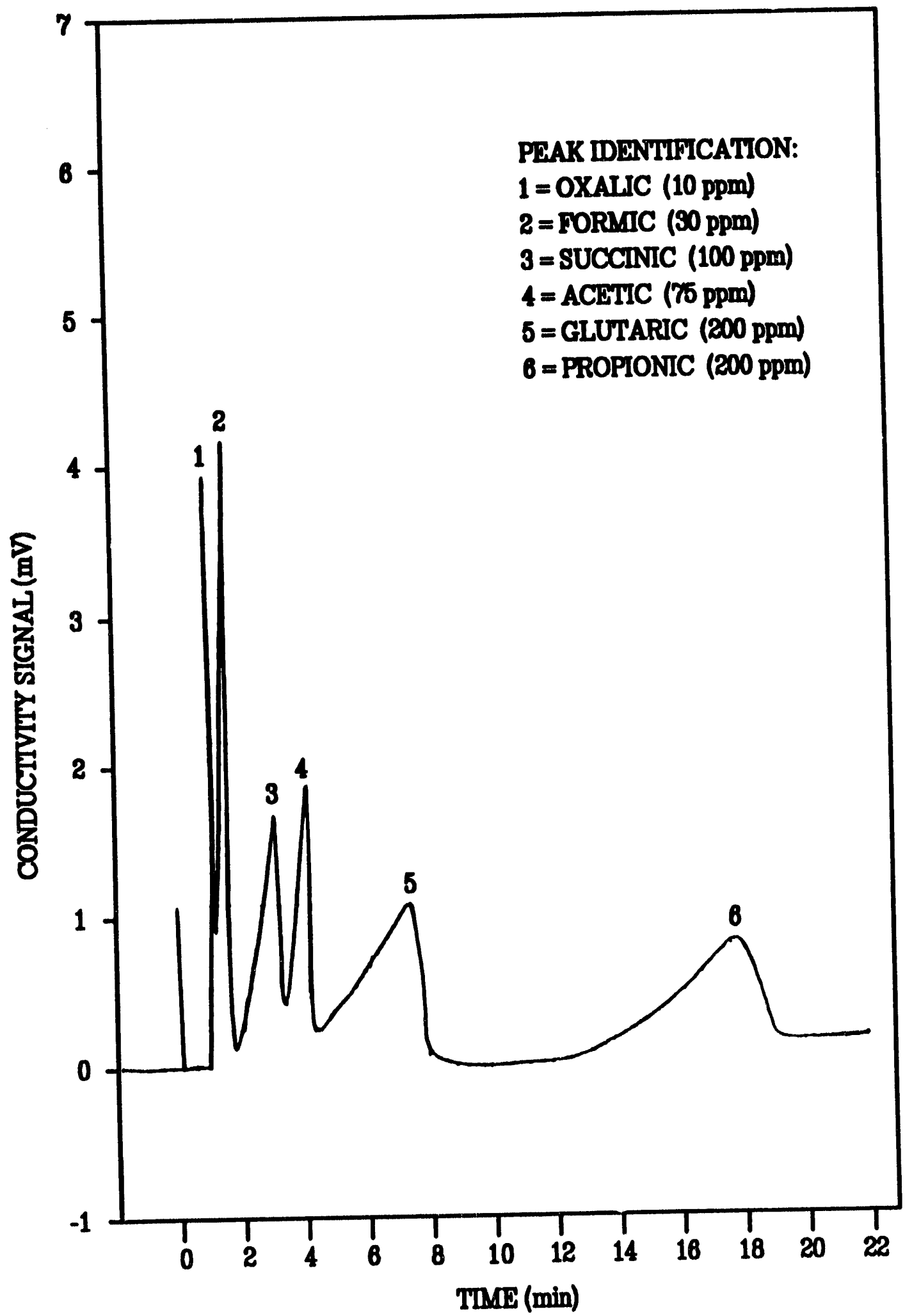


acid elutes before acetic acid. This elution order is not surprising since succinic acid is a dicarboxylic acid and would be largely ionized in water. The second item of interest is the poor peak shape, especially for glutaric and propionic acids. Elution times of butyric and valeric acids were extremely long.

Addition of a small amount of methanol to the eluent improved the peak shape and reduced retention times of the acid solutes. The amount of methanol was increased until an optimized separation was achieved with an eluent containing $60 \%$ methanol / 40\% water as shown in Figure 2. There was a dramatic increase in sensitivity using conductivity detection compared to either UV or RI detection used for previous separations with aqueous sulfuric acid eluents. Injected concentrations range from $1 \mathrm{ppm}$ (formic) to $75 \mathrm{ppm}$ (valeric) with the detector output range set at $3 \mu \mathrm{S}$ full scale. The isocratic separation is complete in less than 6 minutes.

The same optimization procedure was followed and Figure 3 shows the separation of the same 5 acids with an eluent consisting of $40 \%$ ethanol / $60 \%$ water. Using the same detector output range, the injected concentrations range from $0.2 \mathrm{ppm}$ (formic) to $6.0 \mathrm{ppm}$ (valeric) for peaks of similar magnitude.

Figure 4 shows the optimized separation of the 5 alkane carboxylic acids with an eluent consisting of $20 \%$ propanol / $80 \%$ water. The chromatogram displays injected concentrations ranging from $0.25 \mathrm{ppm}$ (formic) to $2.00 \mathrm{ppm}$ (valeric), again using the same detector setting.

Figure 5 shows the optimized separation of the same acid mixture using an eluent of $5 \%$ butanol / $95 \%$ water. Notice that the acids are still essentially baseline 


\section{4}

Figure 2. Chromatographic separation on sulfonated PS-DVB resin column $(150 \times 4.6 \mathrm{~mm})$ of $0.25 \mathrm{meq} / \mathrm{g}$ exchange capacity. Eluent conditions: $60 \%$ methanol/deionized water. Detection is conductivity with an output range of $3 \mu \mathrm{S}$ full scale. Flow rate is $1.0 \mathrm{ml} / \mathrm{min}$. Peaks are as identified. 


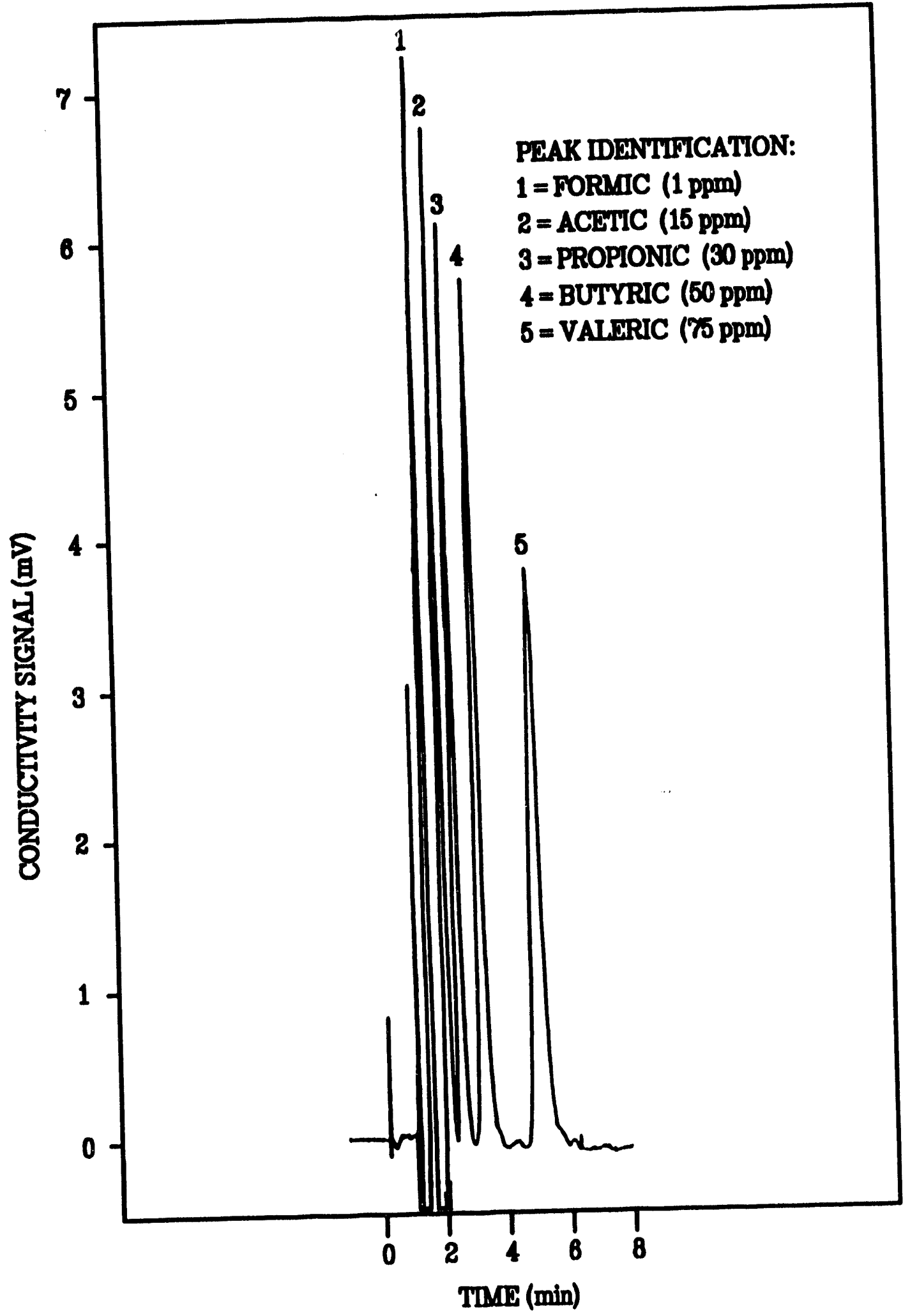


106

Figure 3. Chromatographic separation on sulfonated PS-DVB resin column $(150 \times 4.6 \mathrm{~mm})$ of $0.25 \mathrm{meq} / \mathrm{g}$ exchange capacity. Eluent conditions: $40 \%$ ethanol/deionized water. Detection is conductivity with an output range of $3 \mu \mathrm{S}$ full scale. Flow rate is $1.0 \mathrm{ml} / \mathrm{min}$. Peaks are as identified. 


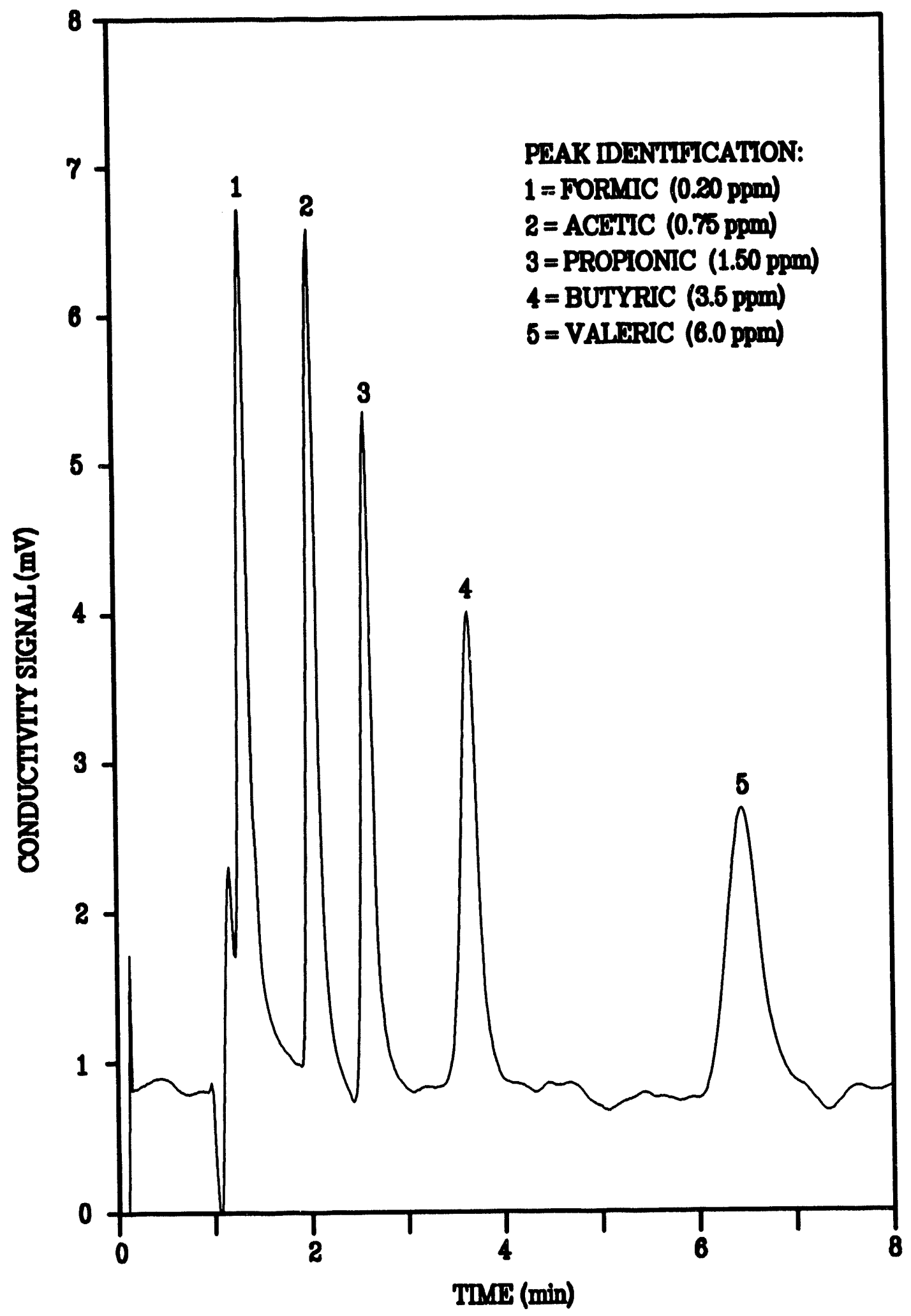




\section{8}

Figure 4. Chromatographic separation on sulfonated PS-DVB resin column $(150 \times 4.6 \mathrm{~mm})$ of $0.25 \mathrm{meq} / \mathrm{g}$ exchange capacity. Eluent conditions: $20 \%$ propanol/deionized water. Detection is conductivity with an output range of $3 \mu \mathrm{S}$ full scale. Flow rate is $1.0 \mathrm{ml} / \mathrm{min}$. Peaks are as identified. 


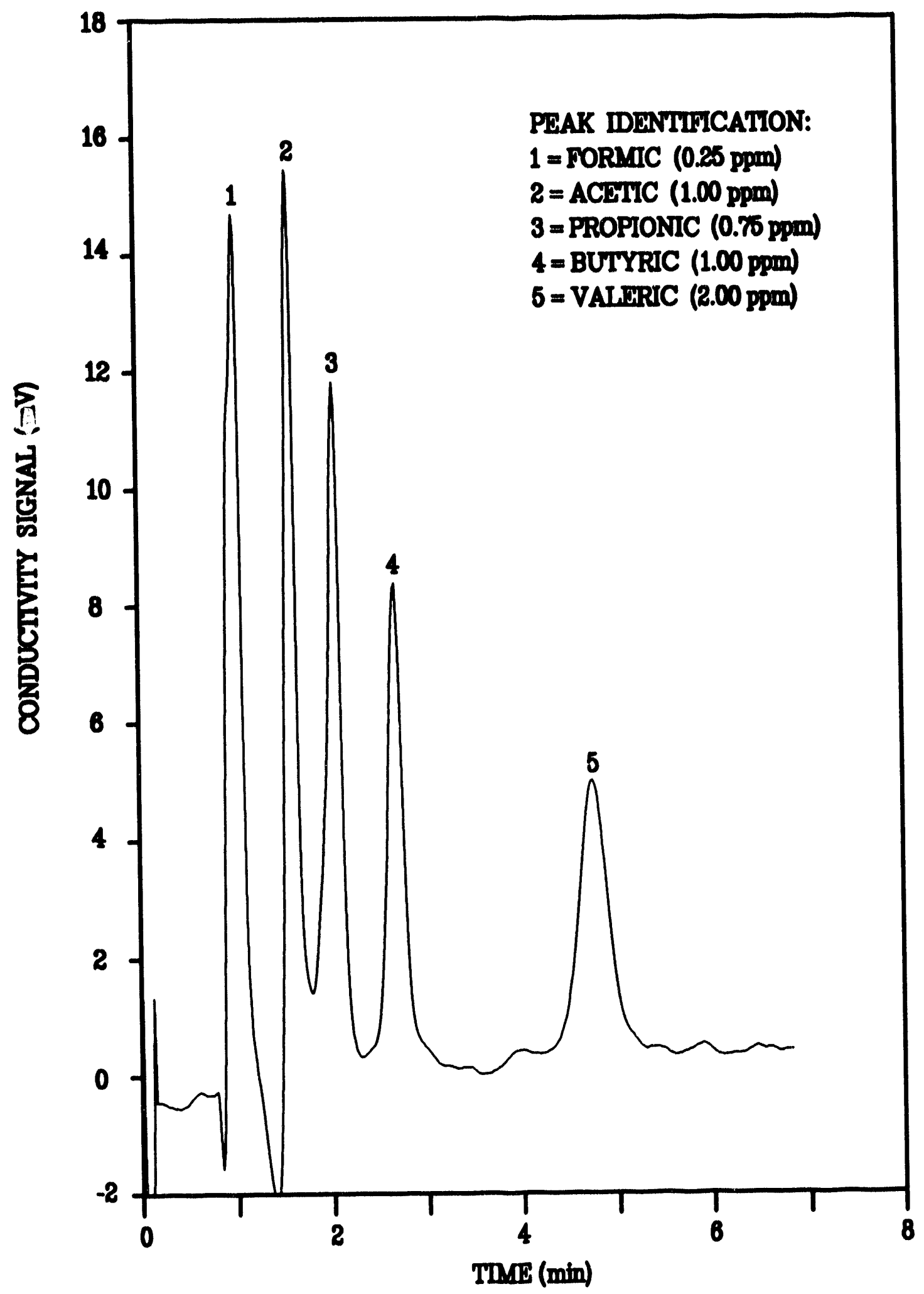


Figure 5. Chromatographic separation on sulfonated PS-DVB resin column $(150 \times 4.6 \mathrm{~mm})$ of $0.25 \mathrm{meq} / \mathrm{g}$ exchange capacity. Eluent conditions: $5 \%$ butanol/deionized water. Detection is conductivity with an output range of $3 \mu \mathrm{S}$ full scale. Flow rate is $1.0 \mathrm{ml} / \mathrm{min}$. Peaks are as identified. 


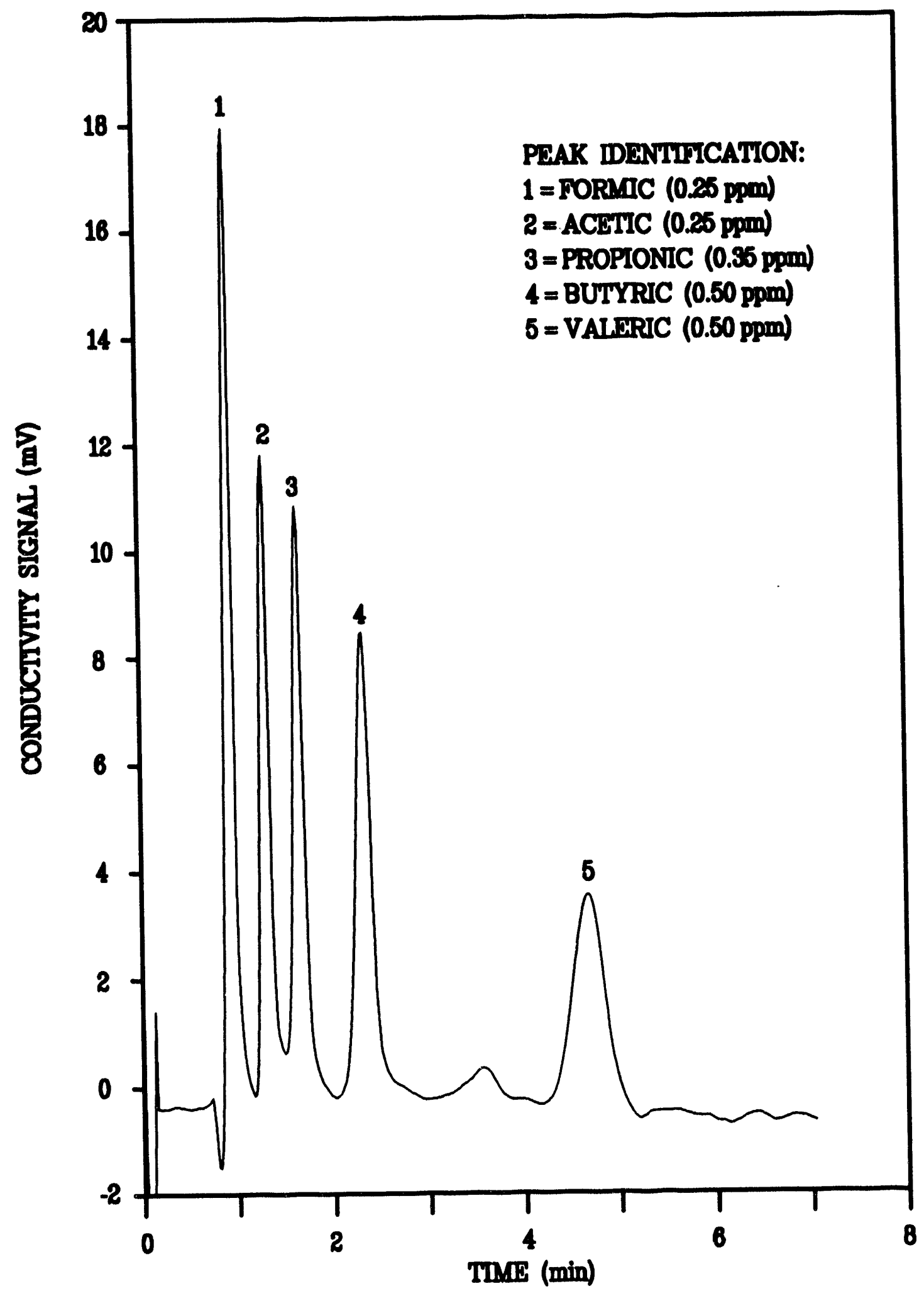


resolved and the separation is complete in approximately 5 minutes. Again, the sensitivity is improved as the injected concentrations range from $0.25 \mathrm{ppm}$ (formic) to $0.50 \mathrm{ppm}$ (valeric).

A few points should be considered with the use of this series of straight chain alcohols. The first is that the detection sensitivity continues to impreve as the chain length of the alcohol increases. In the series of alcohol additives (from methanol to butanol), the sensitivity increases as the percentage of alcohol modifier decreases (the percentage of water increases). The percentage of ionization of the carboxylic acids is higher with the higher percentage of water in the eluent. In the case of a pure water system, a calculation showed the acids to be approximately $69-97 \%$ ionized for the concentratiors injected.

\section{Mechanism of Alcohol Modifiers}

The effect of methanol and ethanol on the chromatographic separations could possibly be explained by stronger solvation of the sample solutes in the liquid eluent. Work done by E.D. Katz, C.H. Lochmüller, and R.P.W. Scott showed that in a binary aqueous system the majority of methanol exists as a water-methanol complex up to a maximum of approximately $60 \%$ methanol (9). In HPLC separations, the capacity factor is thought to be a function of "free" methanol rather than the total methanol content of the eluent. It is thought that the effectiveness of methanol as a modifier is compromised by the hydration of the methanol (i.e. water-methanol "complex") until a percentage of at least $\mathbf{3 0}$ or greater is reached. Presumably, as the chain length of the alcohol increases, the hydration of the alcohol (i.e. water-alcohol "complex" 
formation) would decrease. Therefore, smaller percentages of the higher alcohols exhibit the same effect on the separation. For our carboxylic acid separations with propanol-water and especially with butanol-water, the amount of modifier in the eluent is too small to adequately explain the observed effects on the basis of increased solvation in the liquid phase.

Several authors have found that a portion of the organic solvent in organic-water eluents is adsorbed onto the surface of a bonded-phase silica commonly used in HPLC 10-15). This creates a so-called "third phase" that can alter the partitioning of sample solutes and thereby affect their capacity factors. However, this effect may not be very large with the commonly used eluents such as methanol, acetonitrile or tetrahydrofurane mixed with water. McCormick and Karger (11) found that only $2 \%$ (by volume) of a modifier of somewhat higher molecular weight in a methanol-water or acetonitrile-water eluent was more strongly adsorbed by the stationary phase. They concluded that the concentration of hydrophobic modifiers in the stationary phase is believed to be a significant factor in determining polar selectivity.

Scott and Simpson studied the adsorption of aliphatic alcohols, aldehydes and carboxylic acids in binary mixtures with water by ODS-2 silica (14). They found that the distribution coefficient increases exponentially with the carbon number of the moderator. When usirg an aliphatic moderator having a chain length of 4 or 5 carbon atoms, the surface of a bonded phase could be completely covered with a monolayer. They stated further that the chromatographic characteristics of the surface could be changed by choosing appropriately active groups. 
Adsorption of a layer of alcohol on the polymeric resin surface is believed to explain the dramatic effects observed in our separations of carboxylic acids. A dynamic equilibrium is thought to be established in which some of the alcohol coats the resin surface increasing its hydrophilicity. This reduces the hydrophobic attraction of the resin for the hydrocarbon tail of the carboxylic acids being separated. Figure 6 shows a schematic of this surface modification by the straight-chain alcohol. There also appears to be some pattern in that less alcohol is required to achieve a similar separation in going from methanol to butanol. Butanol has the highest distribution coefficient of the alcohol moderators studied and only a low concentration in the aqueous eluent is needed to coat the resin surface. Partitioning of the various solute acids between the predominately aqueous eluent and the coated resin surface is much different than it is with an uncoated polystyrene surface.

\section{Neutral Aromatic Compounds}

The use of eluent modifiers was expanded to examine a typical reverse phase separation. A few neutral aromatic species were chosen since they would be easily detected by UV absorbance. Figures 7-9 show separations of a mixture of five neutral compounds on a $5-\mathrm{cm}$ sulfonated polymeric resin column at increasing percentages of acetonitrile. The best separation was with an eluent of $60 \% \mathrm{ACN}$.

In hopes of reducing the total amount of required organic solvent, small amounts of a straight-chain alcohol were added to the mobile phase. Since a rather large amount of acetonitrile was necessary to obtain an adequate separation, a larger straight-chain alcohol modifier was selected. It was believed that the smaller straight 


\section{5}

Figure 6. Schematic of butanol dynamic coating on sulfonated polystyrenedivinylbenzene resin surface 


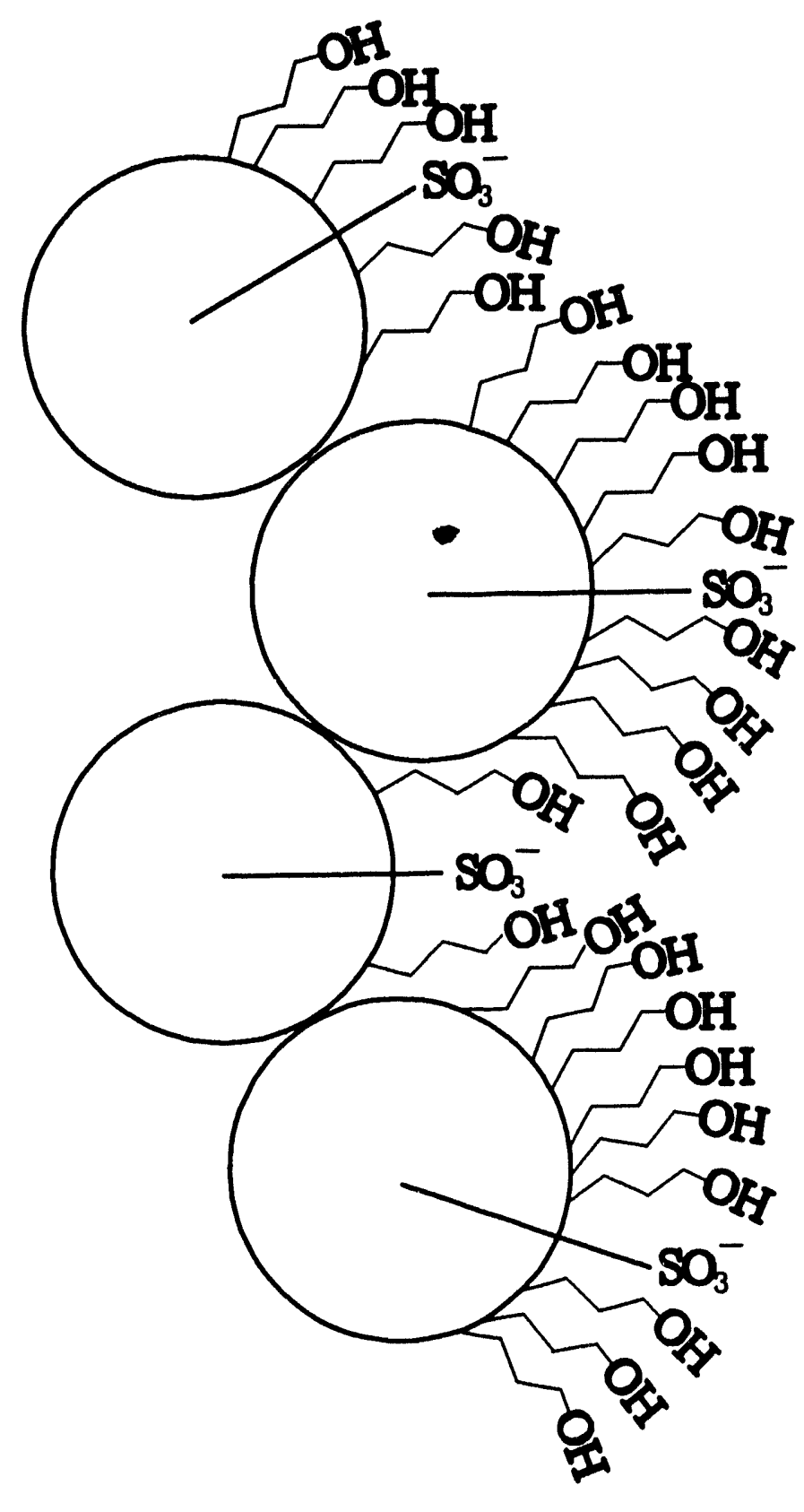

Dynamic Equilibrium: BuOH (resin) $\rightleftarrows \mathrm{BuOH}$ (soln) 


\section{7}

Figure 7. Chromatographic separation on sulfonated PS-DVB resin column $(50 \times 4.6 \mathrm{~mm})$ of $0.25 \mathrm{meq} / \mathrm{g}$ exchange capacity. Eluent conditions: $40 \%$ acetonitrile/deionized water. Detection is UV absorbance at $254 \mathrm{~nm}$ with an output range of 0.001 AUFS. Flow rate is 1.0 $\mathrm{ml} / \mathrm{min}$. Peaks are as identified. 


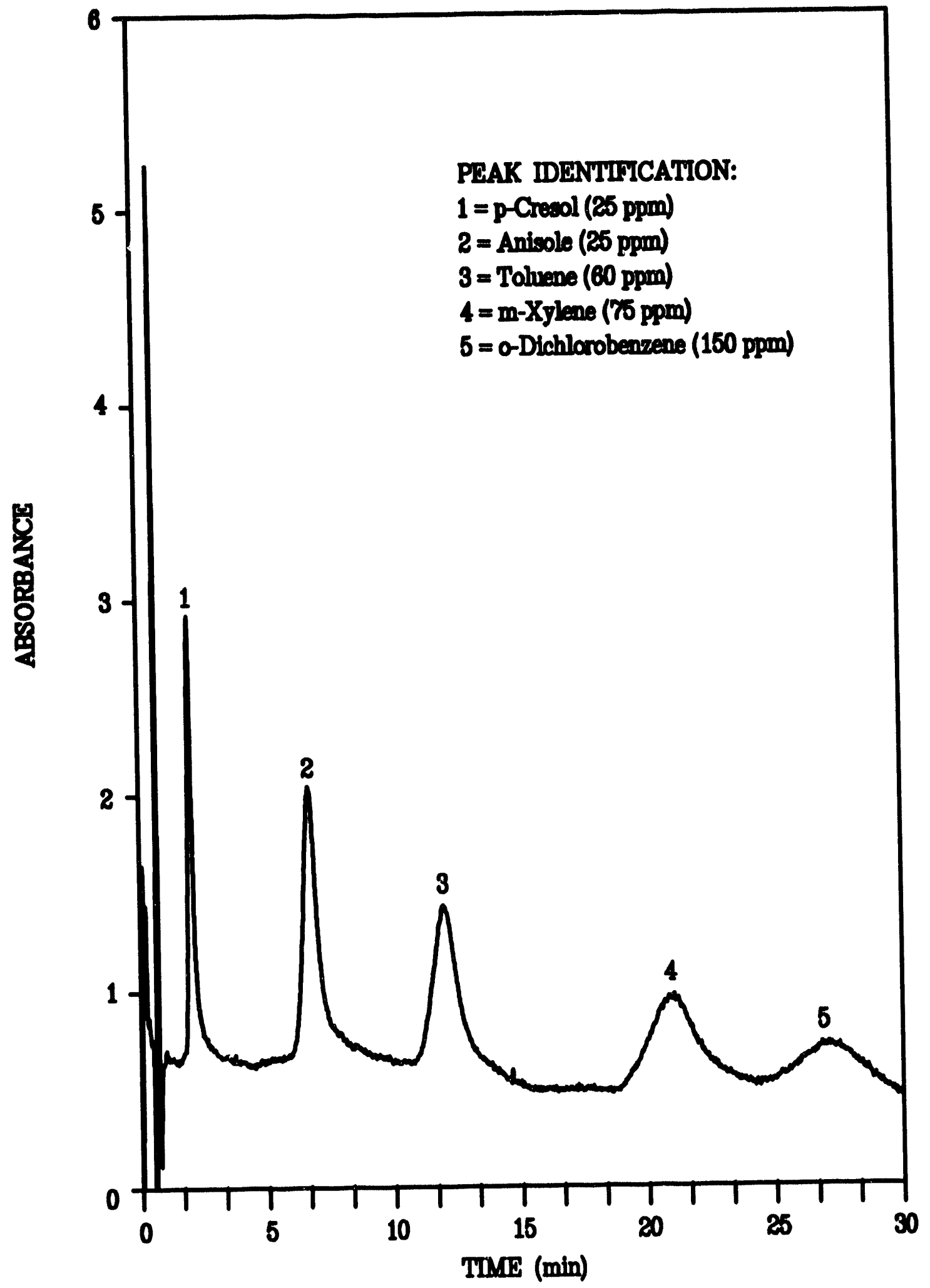


Figure 8. Chromatographic separation on sulfonated PS-DVB resin column $(50 \times 4.6 \mathrm{~mm})$ of $0.25 \mathrm{meq} / \mathrm{g}$ exchange capacity. Eluent conditions: $50 \%$ acetonitrile/deionized water. Detection is UV absorbance at $254 \mathrm{~nm}$ with an output range of 0.001 AUFS. Flow rate is 1.0 $\mathrm{ml} / \mathrm{min}$. Peaks are as identified. 


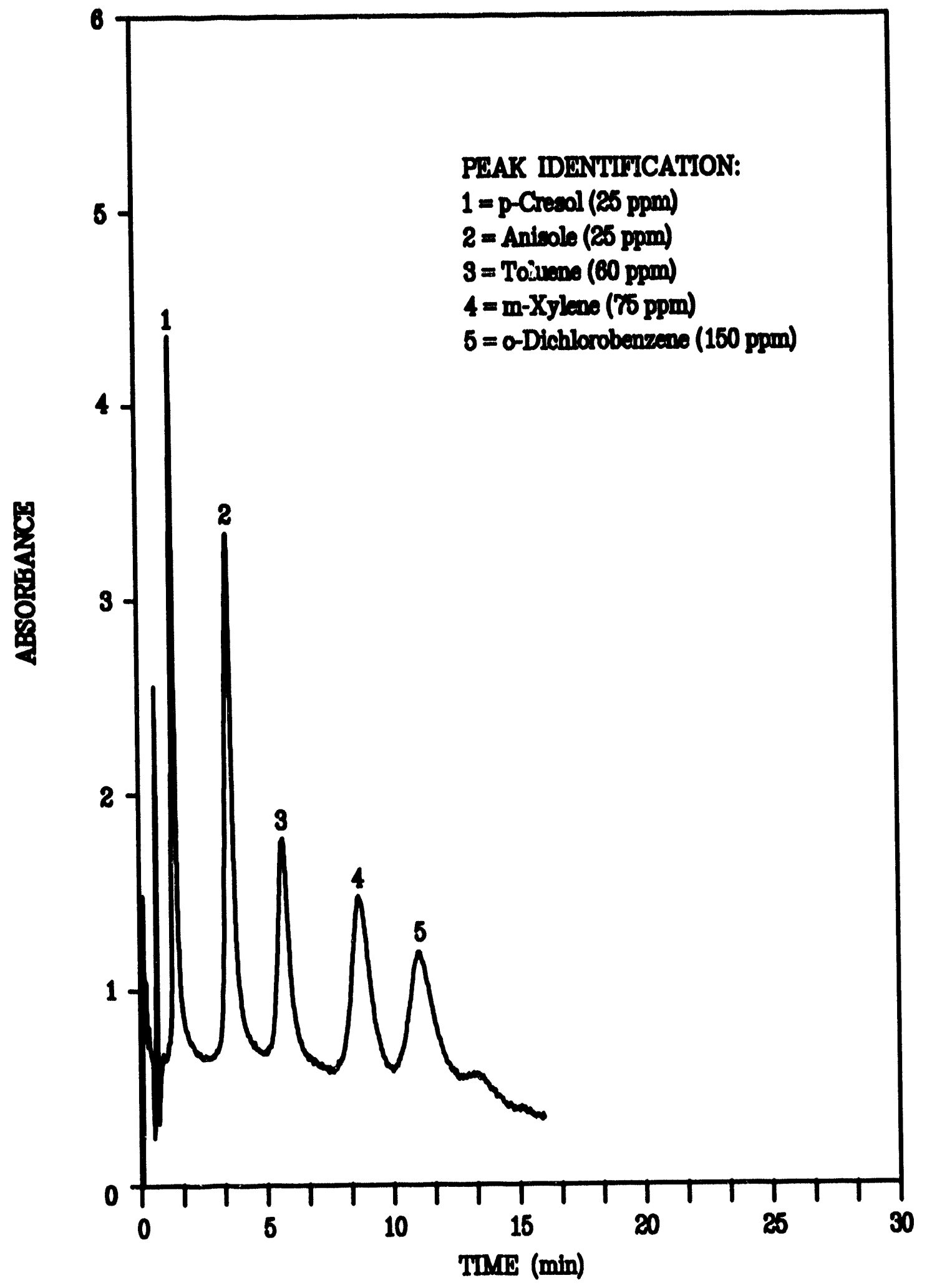


Figure 9. Chromatographic separation on sulfonated PS-DVB resin column $(50 \times 4.6 \mathrm{~mm})$ of $0.25 \mathrm{meq} / \mathrm{g}$ exchange capacity. Eluent conditions: $60 \%$ acetonitrile/deionized water. Detection is UV absorbance at $254 \mathrm{~nm}$ with an output range of 0.001 AUFS. Flow rate is 1.0 $\mathrm{ml} / \mathrm{min}$. Peaks are as identified. 


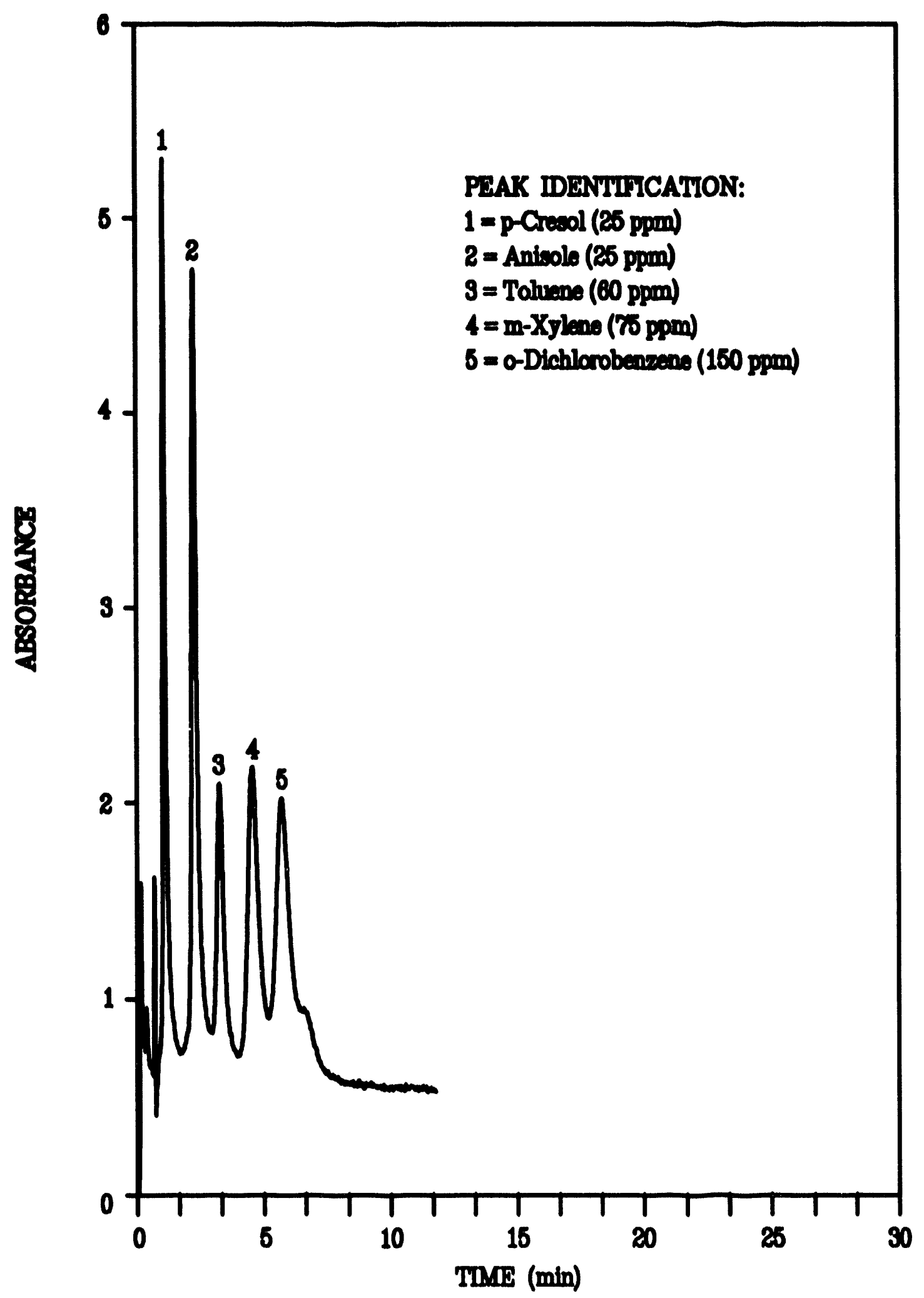


-chain alcohols (methanol through butanol) would be less effective at coating the resin surface when the eluent contained such a large percentage of a strong organic solvent such as acetonitrile.

Figure 10 shows the separation of the neutral mixture with an eluent containing $40 \% \mathrm{ACN} / 5.0 \%$ hexanol/water. For comparison purposes, review the chromatogram shown in Figure $7(40 \% \mathrm{ACN})$. Notice the overall improvement in peak shape and greatly reduced retention times with the eluent containing the hexanol.

Figure 11 shows a separation of the same mixture with an eluent containing $40 \%$ $\mathrm{ACN} / 1.5 \%$ octanol/water. Again, there is a significant improvement in the quality of the chromatogram compared to Figure 7. Further increases in the amount of octanol was prohibited due to its low solubility. The amount shown (1.5\%) was near the solubility maximum in $40 \%$ acetonitrile.

Figure 12 shows the separation of the same mixture with an eluent containing $40 \% \mathrm{ACN} / 0.3 \%$ decanol/water. A small improvement was noted but not quite as dramatic as with the hexanol. This was the maximum amount of decanol that would dissolve in $40 \%$ acetonitrile.

There are a couple of important observations to be made here. First, longer column equilibration times are required as the percentage of alcohol modifier decreases. In the case of the decanol, the very small amount $(0.3 \%)$ present in the eluent requires several times longer to fully equilibrate and establish a dynamic equilibrium with the resin matrix. Second, the effect of the alcohol eluent modifiers seems more pronounced when applied to compounds which are more polar such as the 
124

Figure 10. Chromatographic separation on sulfonated PS-DVB resin column $(50 \times 4.6 \mathrm{~mm})$ of $0.25 \mathrm{meq} / \mathrm{g}$ exchange capacity. Eluent conditions: $40 \%$ acetonitrile $+5 \%$ hexanol/deionized water. Detection is UV absorbance at $254 \mathrm{~nm}$ with an output range of 0.001 AUFS. Flow rate is $1.0 \mathrm{ml} / \mathrm{min}$. Peaks are as identified. 


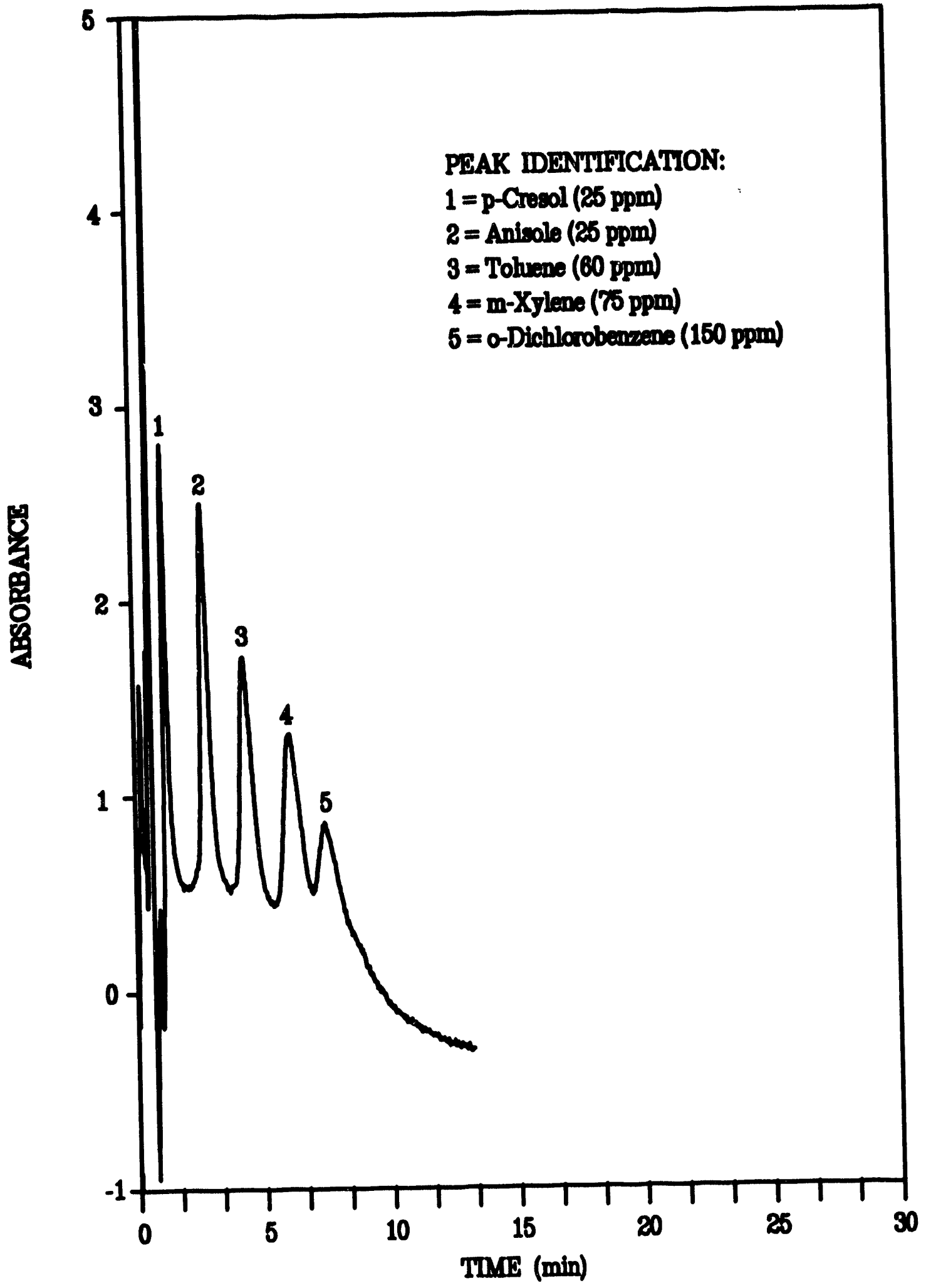


Figure 11. Chromatographic separation on sulfonated PS-DVB resin column $(50 \times 4.6 \mathrm{~mm})$ of $0.25 \mathrm{meq} / \mathrm{g}$ exchange capacity. Eluent conditions: $40 \%$ acetonitrile $+1.5 \%$ octanol/deionized water. Detection is UV absorbance at $254 \mathrm{~nm}$ with an output range of 0.001 AUFS. Flow rate is $1.0 \mathrm{ml} / \mathrm{min}$. Peaks are as identified. 


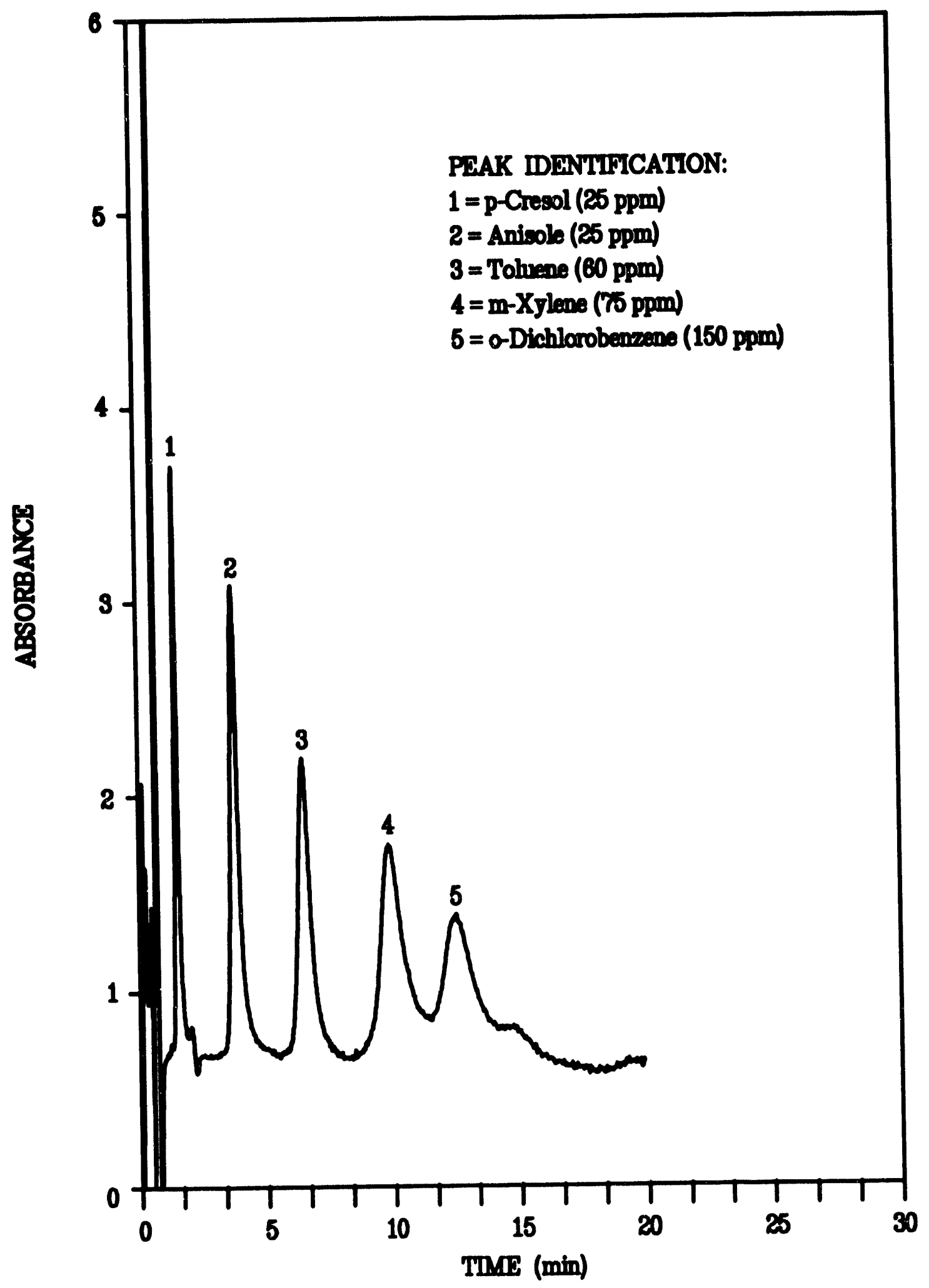


128

Figure 12. Chromatographic separation on sulfonated PS-DVB resin column $(50 \times 4.6 \mathrm{~mm})$ of $0.25 \mathrm{meq} / \mathrm{g}$ exchange capacity. Eluent conditions: $40 \%$ acetonitrile $+0.3 \%$ decanol/deionized water. Detection is UV absorbance at $254 \mathrm{~nm}$ with an output range of 0.001 AUFS. Flow rate is $1.0 \mathrm{ml} / \mathrm{min}$. Peaks are as identified. 


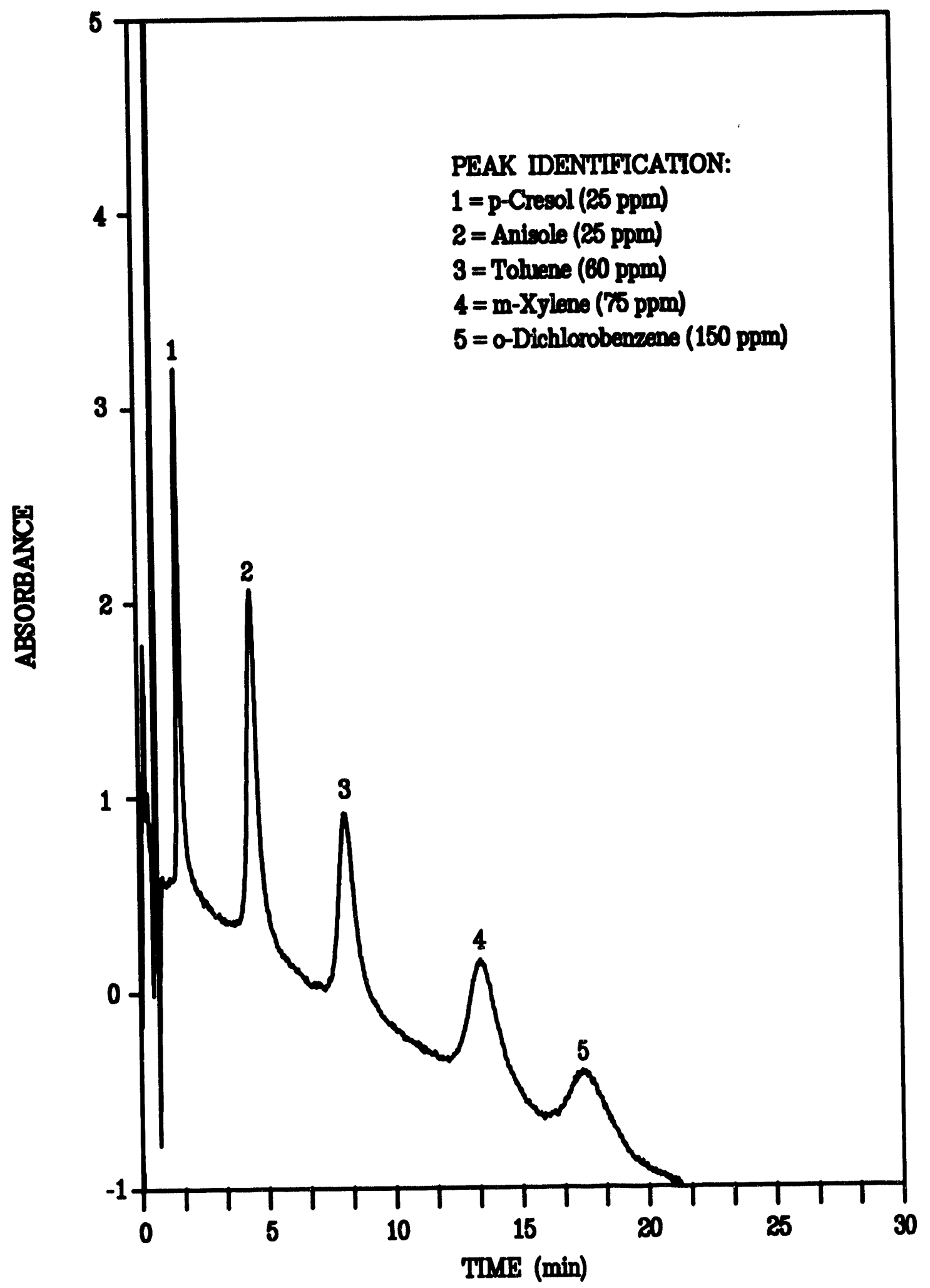


carboxylic acids. A small percentage of a straight-chain alcohol in an otherwise aqueous eluent has a very dramatic effect. The alcohol modifiers have a similar effect when applied to the neutral aromatic species, but due to the highly hydrophobic nature of these analytes, a large amount of organic solvent is still required. Perhaps there are other types of modifiers containing different functional groups that will have a larger impact on improving the separation.

\section{Semi-Permanent Coatings with Alcohol Modifiers}

In the previously discussed work, the various alcohol modifiers added to the eluent would establish a dynamic equilibrium with the polymeric resin matrix. When the idea was expanded to larger straight-chain alcohols, such as decanol, it was found that the dynamic equilibrium was established much more slowly due to the low percentage of alcohol that could be used. We decided to try coating a large straightchain alcohol on to the polymeric resin and use a predominantly aqueous eluent for the separation. The long-chain alcohol was dissolved in a solvent that was mainly organic (i.e. $1.9 \%$ dodecanol in $75 \%$ methanol) and passed through the analytical column at a slow flow rate for several hours. The column was then flushed with deionized water and equilibrated with the water eluent. The idea was that the dodecanol would form a coating on the resin surface which should be very stable (by hydrophobic attraction) when using a mostly aqueous eluent. Figure 13 shows the separation of formic through butyric acids using only water as an eluent and conductivity detection on the column treated with dodecanol as described above. For comparison, please refer to Figure 1. This chromatogram shows separation of formic, acetic, and propionic acids under the 
131

Figure 13. Chromatographic separation on sulfonated PS-DVB resin column $(150 \times 4.6 \mathrm{~mm})$ of $0.25 \mathrm{meq} / \mathrm{g}$ exchange capacity after coating with dodecanol. Eluent conditions: deionized water. Detection is conductivity with an output range of $3 \mu \mathrm{S}$ full scale. Flow rate is $1.0 \mathrm{ml} / \mathrm{min}$. Peaks are as identified. 


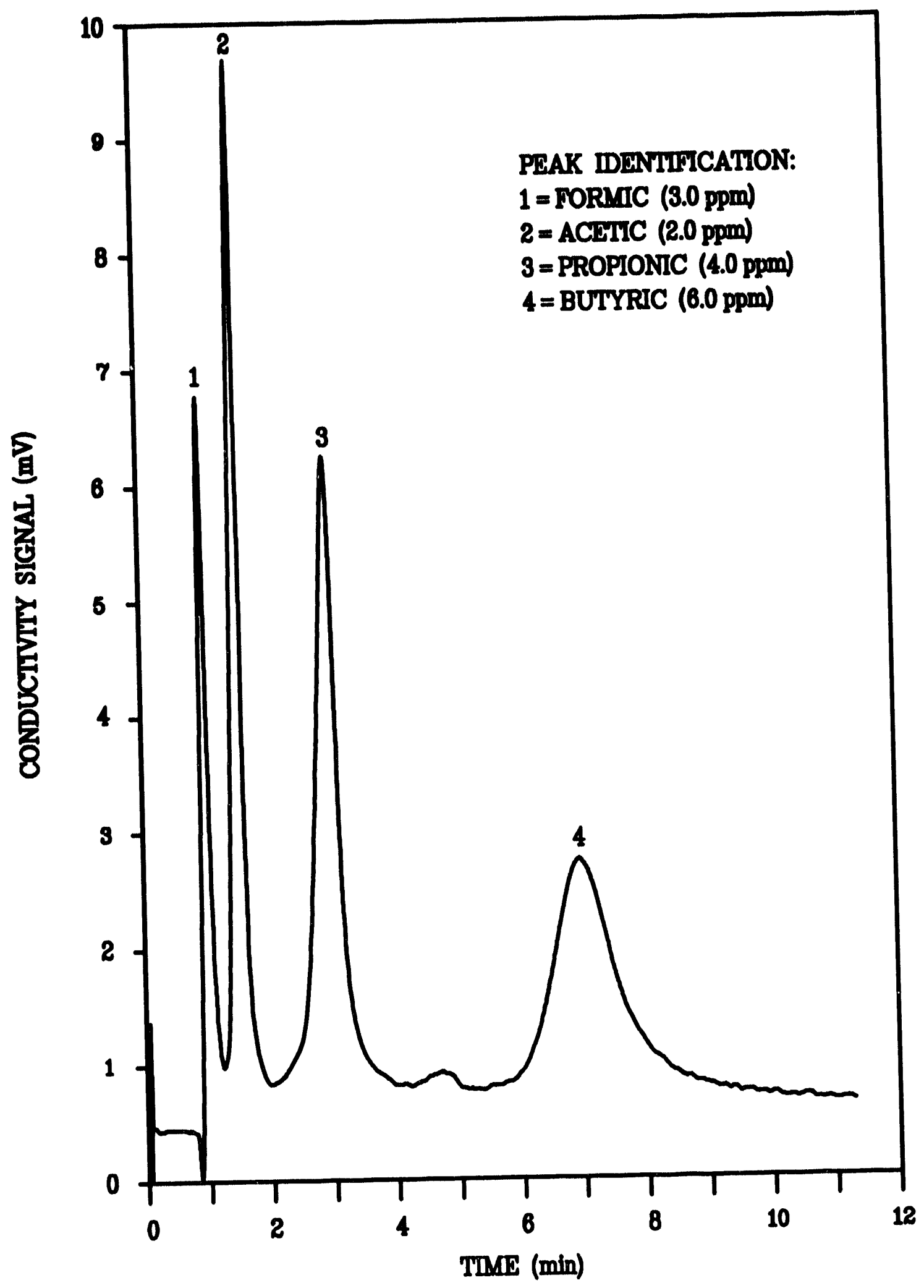


same conditions and using the same column prior to the dodecanol treatment. The surface properties of the resin have been significantly altered by the dodecanol coating. The resin appears to be much more hydrophilic with the alcohol coating as indicated by the decreased retention times and sharper peaks for the analyte acids. This is especially noticed for the later eluting acids that have a more hydrophobic nature. For example, the retention time for propionic acid changes from around $18 \mathrm{~min}$ to $3 \mathrm{~min}$ following the treatment of the column with the dodecanol. The peak shape is also greatly improved.

The durability of the alcohol coating will be a very important factor. The column was flushed for 6 hours at $1.0 \mathrm{ml} / \mathrm{min}$ with $10 \%$ methanol/water with no noticeable loss of the dodecanol coating. This was determined by injection of a standard mixture before and after the flushing period. In addition, the column was flushed for 24 hours with deionized water at the same flow rate. After 24 hours, there appears to be no degradation in the quality of the separation.

\section{Effect of Carbonic Acid on Retention Mechanism}

\section{Dynamic Alcohol Coating}

Our separations up to this point were performed on lightly sulfonated polymeric resins. The small amount of sulfonic acid groups makes the resin surface more hydrophilic so that analyte species can approach and interact mole readily with the resin matrix. It is conceivable that the sulfonic acid substituent may also affect the separation of analytes by converting the carboxylate anions to molecular carboxylic acid at the resin surface. This idea was tested by performing the separations under identical 
conditions but using an underivatized resin column. The experiments using an underivatized resin should yield additional information as to the role of the sulfonic acid group in the separation mechanism. Figure 14 shows that an unfunctionalized polymeric resin can be used for the separation of the carboxylic acid mixture. Small differences between the two types of resins were noted but could be attributed to differences in the hydrophilic properties of the underivatized and the chemically modified resins. These results suggest that the sulfonic acid group does not play a vital role in the separation process.

In general, greater retention and better separations are obtained using a resin with polar substituents such as sulfonate or carboxylate. A very small amount of functionalization (i.e. low capacity) has been shown by our group and others to provide optimal retention of organic species by hydrophobic attraction. The very small amount of polar substituents makes the surface of the resin more hydrophilic so that analyte species may approach and interact with the resin matrix more readily.

Chromatographic separation of the alkane carboxylic acids was successful on columns packed with either an underivatized macroporous polymeric resin (PS-DVB) or the same resin which had been lightly sulfonated. This raised some interesting questions regarding the mechanism of retention to permit chromatographic separation. For example, why are we getting effective chromatographic separation when the acids are calculated to be mostly ionized at the low concentrations used? If the acids exist predominantly as anions, one would expect them to be strongly solvated by the aqueous eluent leading to fast elution. In fact, however, the acids show reasonable retention 


\section{5}

Figure 14. Chromatographic separation on underivatized PS-DVB resin column $(150 \times 4.6 \mathrm{~mm})$. Eluent conditions: $4 \%$ butanol/ deionized water. Detection is conductivity with an output range of $3 \mu \mathrm{S}$ full scale. Flow rate is $1.0 \mathrm{ml} / \mathrm{min}$. Peaks are as identified. 


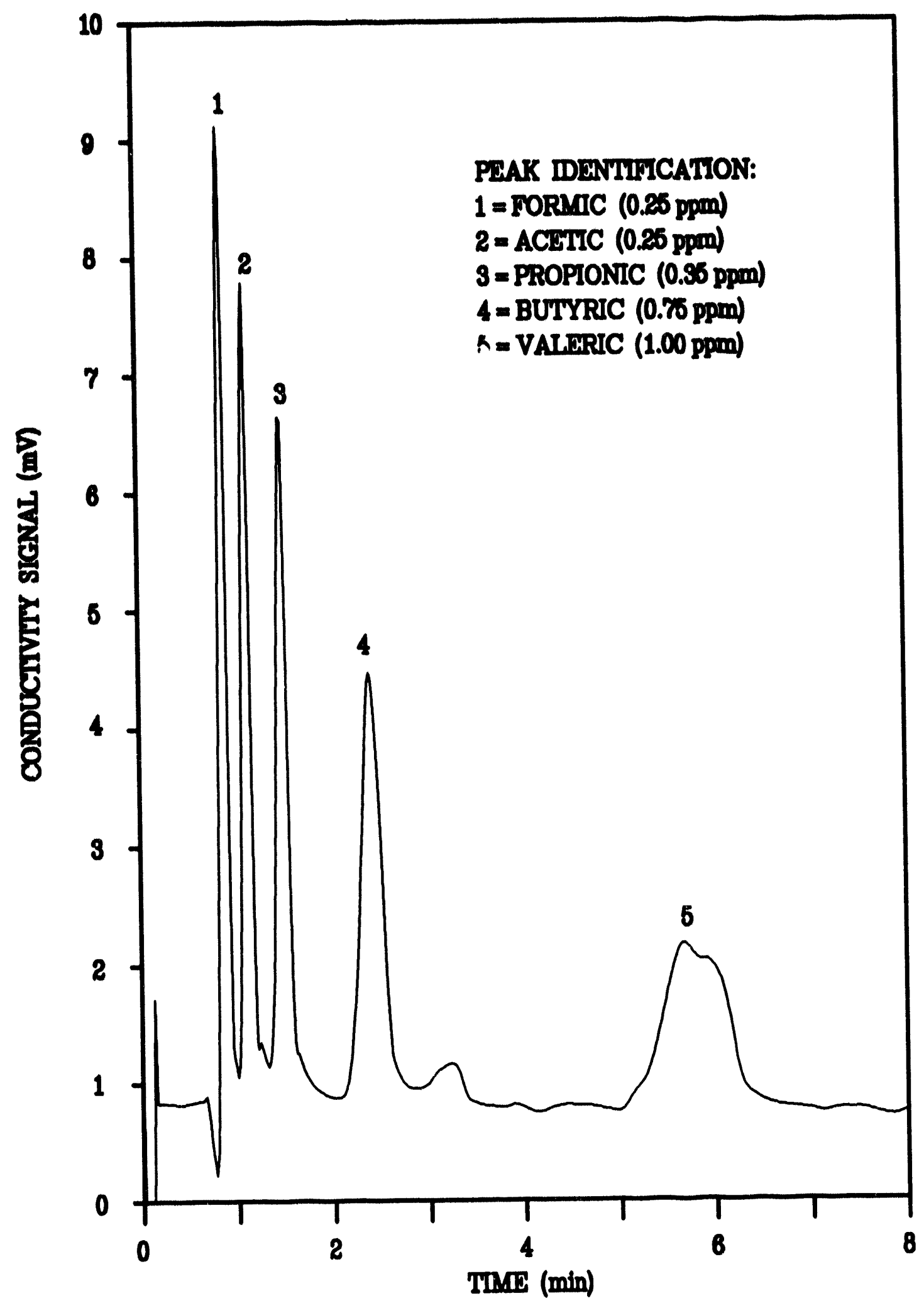


allowing for separation with the elution order based on the length of the hydrocarbon chain. This seems to approximate previous separations in which a mineral acid (1 mM $\mathrm{H}_{2} \mathrm{SO}_{4}$ ) was added to the eluent to repress ionization of the analyte acids so that they would exist mostly as molecular acids.

In an effort to find additional information about the retention mechanism, we investigated the chromatographic behavior of sodium salts of these alkane carboxylic acids. An underivatized column was chosen since we wanted to avoid introducing any source of acid into our system. A simple ion exchange would take place on a sulfonated resin converting the salt to the acid. Figure 15A shows the separation of formic acid, acetic acid, propionic acid. and sodium butyrate on an underivatized resin column using a $4 \%$ butanol/water eluent. Figures $15 B$ and $15 \mathrm{C}$ show the separation of the sodium salts of these same acids at the same concentrations. Comparison of the chromatograms reveals no great differences. The salts behave almost the same as the acids possibly indicating that the salts are being converted to acids.

In reviewing our complete chromatographic system, we could find no source of acid other than carbonic acid (or $\mathrm{CO}_{2}$ ). Since the analyte acids are weaker carboxylic acids, it is possible that carbonic acid could affect the retention mechanism. It was also shown in previous work that carbonic acid has a substantial capacity factor (6). In other words, the carbonic acid spends a significant amount of time residing on the surface of the polymeric resin. In this particular case, the carbonic acid could be a natural impurity that works to our advantage. 
Figure 15. Successive injections of $\left.\mathrm{A}_{0}\right)$ formic acid $\left(3.25 \times 10^{-6} \mathrm{M}\right)$, acetic acid $\left(6.66 \times 10^{-6} \mathrm{M}\right)$, propionic acid $\left(1.08 \times 10^{-5} \mathrm{M}\right)$, and sodium butyrate $\left(9.08 \times 10^{-6} \mathrm{M}\right) ; \mathrm{B}$. and $\mathrm{C}$.) sodium formate, sodium acetate, sodium propionate, and sodium butyrate in the same respective concentrations. Separations performed on underivatized PS-DVB resin column $(150 \times 4.6 \mathrm{~mm})$. Eluent conditions: $4 \%$ butanol/ deionized water. Detection is conductivity with an output range of $10 \mu \mathrm{S}$ full scale. Flow rate is $1.0 \mathrm{ml} / \mathrm{min}$. The elution order of the analytes is as listed. 


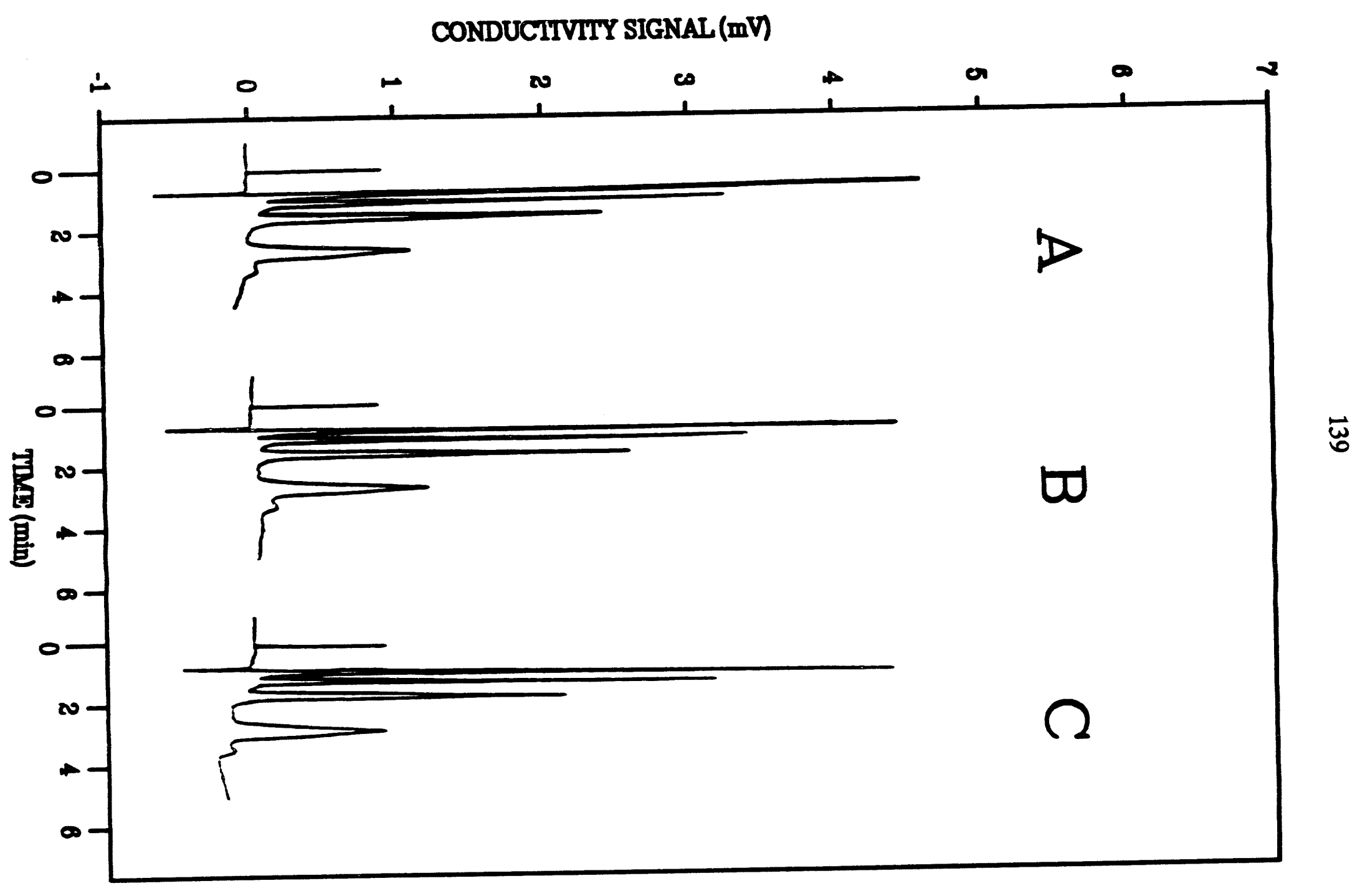


A series of experiments were performed to help determine the effects of the carbonic acid in the eluent. First, approximately $100 \mathrm{ml}$ of the eluent mixture (4\% butanol/water) was placed in a beaker and the $\mathrm{pH}$ was measured. Initially, the $\mathrm{pH}$ was close to neutral ( $\mathrm{pH} 6.8)$. With time, however, the $\mathrm{pH}$ gradually drifted toward more acidic $\mathrm{pH}$ values. As the solution stands exposed to the atmosphere, one would expect continuous absorption of carbon dioxide and a more acidic $\mathrm{pH}$ with the resultant formation of carbonic acid. Even though our eluent is sparged and kept under a blanket of helium, it would be very difficult to completely remove all carbonic acid. Any amount present in the water-alcohol mixture will equilibrate with the resin.

The next experiments were performed after removing the analytical column and connecting the conductivity detector directly to the injection port. This configuration allowed us to do some flow injection analyses. Figure 16 shows the detection signal obtained from the equal concentration injections of formic acid (A), sodium formate (B), and sodium formate after treatment with carbon dioxide (dry ice) (C). The magnitude of the conducitvity signal was lower for equal concentration of the salt compared to the acid. This is expected based on the theoretical limited equivalent conductances for the two species (i.e. $\mathrm{H}^{+}=350, \mathrm{Na}^{+}=50$, and the formate anion is the same for both). Notice, however, that the magnitude of the signal for the salt treated with carbon dioxide closely approximates the signal obtained for the injected acid. This suggests that there is conversion of the salt to the acid species by the carbonic acid present in the eluent. Similar results were obtained when the same experiment was performed on the other carboxylic acids such as acetic and propionic 
141

Figure 16. Three successive injections of A.) formic acid $\left(3.25 \times 10^{-6} \mathrm{M}\right)$, B.) sodium formate $\left(3.25 \times 10^{-6} \mathrm{M}\right)$, and C.) sodium formate $(3.25 \times$ $\left.10^{-6} \mathrm{M}\right)$ after treatment with carbon dioxide. Flow injection analyses are performed with the detector connected directly to the injection valve. Eluent conditions: $4 \%$ butanol/ deionized water. Detection is conductivity with an output range of $30 \mu \mathrm{S}$ full scale. Flow rate is $1.0 \mathrm{ml} / \mathrm{min}$. 
<smiles></smiles> 
acids.

Additional experiments were needed to provide information as to whether the effect of the carbonic acid is a solution or a surface phenomenon. Closer examination of previous chromatograms using various organic mobile phase modifiers, showed a small consistent system peak at approximately $3.5 \mathrm{~min}$. Depending on the retention times of the analyte acids, this system peak can show up as a small shoulder on another peak or possibly coelute in some cases. The system peak became very noticeable when the amplification factor of the acquired data was increased. Figure 17 shows a chromatogram in which the system peak is very evident. The conditions are the same as previous chromatograms except for the larger signal amplification. The system peak was identified by injection of only carbonic acid which gives a peak at the same retention time. The origin of the system peak is believed to be the carbonic acid that elutes after disruption of the established equilibrium which follows the injection of the acid mixture.

Using the same system configuration as above, one additional component was added. An anion exchange column in the hydroxide form was placed in-line between the pump and the injection valve. In this manner, the anex column should act as a filter to remove any carbonic acid from the mobile phase prior to the separation column. Figure 18A shows the separation of formic through butyric acids under established conditions prior to the insertion of the anex column. Notice the background conductance of $904 \mathrm{nS}$. Flow was then stopped and the anex column was placed in-line. As flow was resumed, injections were made at timed intervals and 
144

Figure 17. Chromatographic separation on sulfonated PS-DVB resin column $(150 \times 4.6 \mathrm{~mm})$ of $0.25 \mathrm{meq} / \mathrm{g}$ exchange capacity. Eluent conditions: $4 \%$ butanol/deionized water. Detection is conductivity with an output range of $3 \mu \mathrm{S}$ full scale. Amplification factor is 3000. Flow rate is $1.0 \mathrm{ml} / \mathrm{min}$. Peaks are as identified. 


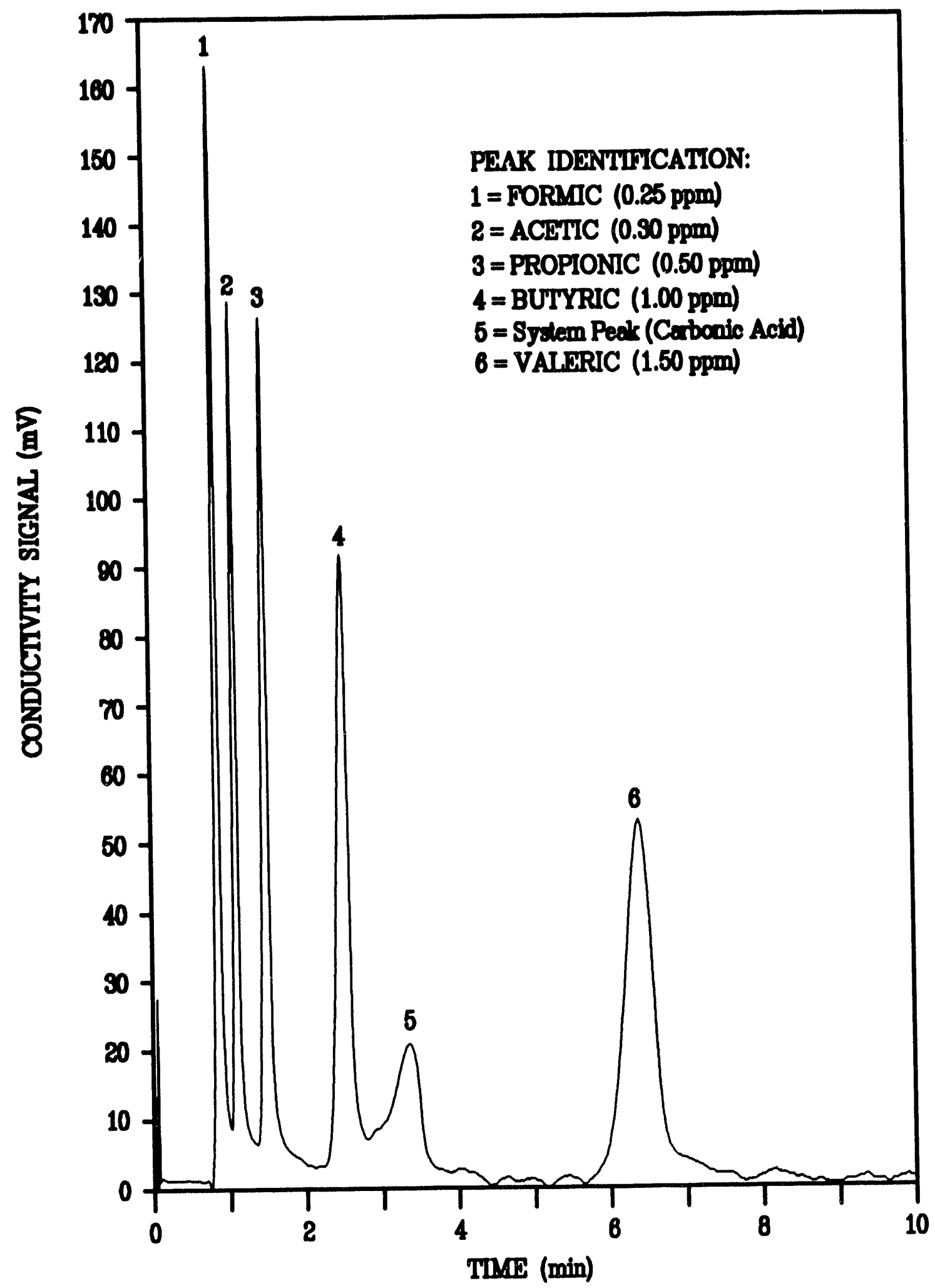




\section{6}

Figure 18. Chromatographic separation on sulfonated PS-DVB resin column $(150 \times 4.6 \mathrm{~mm})$ of $0.25 \mathrm{meq} / \mathrm{g}$ exchange capacity. A.) initial separation, B.) separation after 30 mins. with anion exchange column (hydroxide form) in-line between pump and injection valve, and C.) separation 30 mins. after removal of anion exchange column (initial configuration). Eluent conditions: $4 \%$ butanol/deionized water. Detection is conductivity with an output range of $3 \mu \mathrm{S}$ full scale. Flow rate is $1.0 \mathrm{ml} / \mathrm{min}$. Peaks are as identified. 


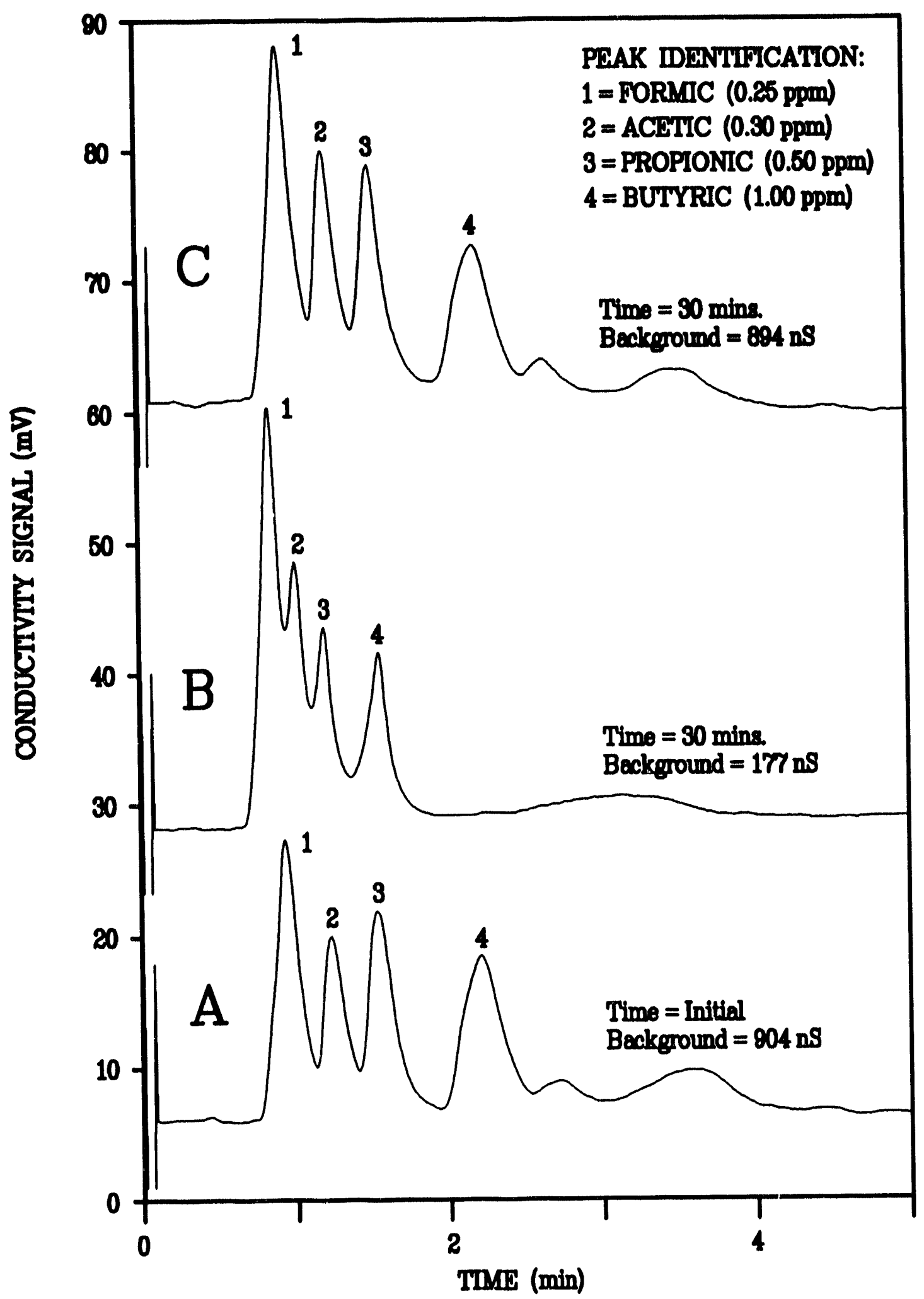


background conductances were recorded. Considering the dead volume of the system, the mobile phase should be free of carbonic acid aftei a couple of minutes. The background conductance signal, however, decreased more gradually indicating that the carbonic acid was slowly being washed off the column. The background signal stabilized after 30 minutes to a significantly lower value of $177 \mathrm{nS}$. Figure 18B shows the separation of the same mixture at this time. The resolution of the acid analytes was noticeably decreased. This indicates that the carbonic acid plays a role in the retention mechanism of the weak-acid analytes. As the carboxylic acids approach the resin surface pre: minantly as anions, the carbonic acid could temporarily convert them to the molecular acids. This would then help facilitate separation of these alkane carboxylic acids based on the length of the hydrocarbon portion of the molecules. Finally, the anex column was removed from the system. The background conductance gradually increased toward the original value. Figure $18 \mathrm{C}$ shows a separation of the same mixture when the background had stabilized 30 minutes after removal of the anex column. The resolution of the acid species and the background signal had returned to the same value as the original chromatogram.

Next, the behavior of alkane sulfonic acids and their respective sodium salts was investigated. Based on the success of the separation of the alkane carboxylic acids, it was thought that a similar series of sulfonic acids could also be separated. No separation was achieved for the alkane sulfonic acids or their sodium salts. All acids in the series eluted with the same retention time. This was not surprising, considering the strength of these acids. The sulfonic acid is a much stronger acid than the 
carboxylic acid a.d consequently is not going to be affected in the same manner by the presence of the carbonic acid. The alkane sulfonic acids will be highly ionized and have very fast, chromatographic elution. This was evidenced by the identical retention times irregardless of the length of the hydrocarbon portion of the molecule. This was further confirmation that the role of carbonic acid on retention mechanism was a phenomenon unique to the weaker carboxylic acids.

\section{Semi-Permanent Alcohol Coating}

The effect of carbonic acid was also investigated on the dodecanol-coated polymeric resin column. A deionized water eluent was passed through the column for 12 hours. The eluent was allowed to sit exposed to the atmosphere. Over the 12 hour period, the background conductance roughly doubled (1.5 $\mu \mathrm{S}$ to $3.0 \mu \mathrm{S})$ and injection of the standard mixture resulted in a fluctuating conductivity signal. After a period of time, the signal would again stabilize. As expected, the eluent took up increasing amounts of carbon dioxide from the atmosphere. As time passed, the increased carbonic acid content in the eluent gave an increased background signal. Similar signal fluctuations were seen in experiments where carbonic acid was injected in large amounts. After the column becomes heavily loaded with the carbonic acid, an injection could lead to severe disruption of the established equilibrium until it stabilizes at a new level.

Freshly deionized water was used to prepare an eluent which was then equilibrated for approximately one hour. The background conductance returned to near the original value of $1.5 \mu \mathrm{S}$ and a separation of the standard mixture returned to 
normal. In a final experiment, the column was again flushed with deionized water for 12 hours. This time, the eluent was degassed and kept under a blanket of helium. The background conductance was much lower ( $271 \mathrm{nS})$ and remained at that level for the entire 12-hour period. Figure 19 shows a separation of the standard mixture at the end of the 12 hour period. Notice the slightly different retention times and resolution when compared to Figure 13. Presumably, this is due to the decreased amount of carbonic acid in the system. Remember that the retention time of propionic acid was $18 \mathrm{~min}$ with water as an eluent on the same column prior to coating.

The effect of the carbonic acid is an interesiing situation. The presence of the carbonic acid appears beneficial for separation of the alkane carboxylic acids, when it is in moderate and controlled quantities. This does not present a problem for most HPLC systems which are equipped with a degassing module. For normal operation, the alcohol/water eluent can be prepared without any special treatment. Whatever small amount of carbonic acid that exists naturally in the alcohol or water will establish an equilibrium between the mobile and stationary phases. This proves to be sufficient for separation of the alkane carboxylic acids. It appears that a reasonable estimation of the carbonic acid content can be obtained by monitoring the background conductance signal. The total amount of carbonic acid remains constant when the eluent is protected from contact with the atmosphere as is the case with helium degassing. 


\section{1}

Figure 19. Chromatographic separation on sulfonated PS-DVB resin column $(150 \times 4.6 \mathrm{~mm})$ of $0.25 \mathrm{meq} / \mathrm{g}$ exchange capacity after coating with dodecanol. Eluent conditions: deionized water for 12 hours under helium blanket. Detection is conductivity with an output range of $3 \mu \mathrm{S}$ full scale. Flow rate is $1.0 \mathrm{ml} / \mathrm{min}$. Peaks are as identified. 


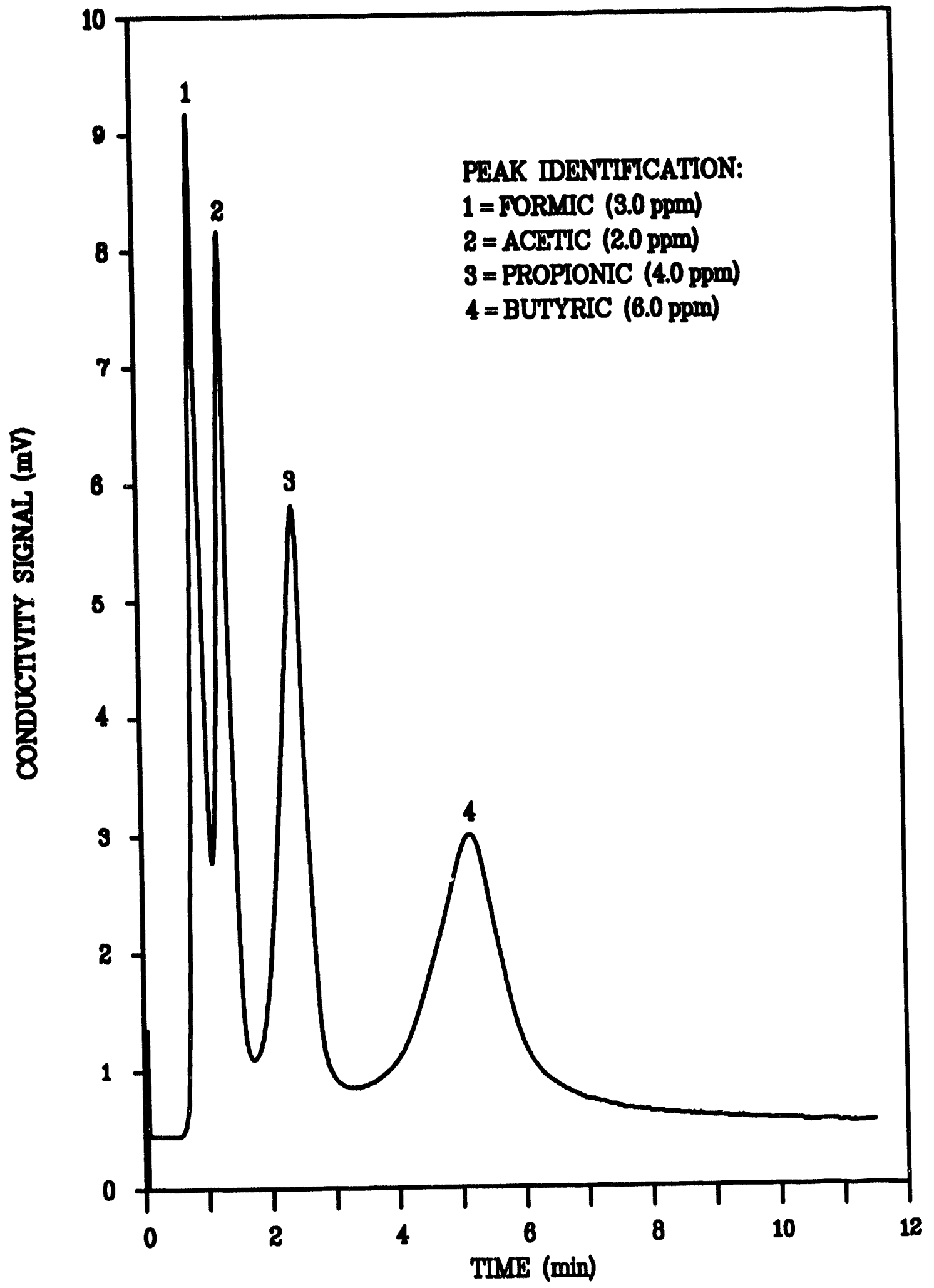




\section{Quantitative Chromatographic Determinations}

A series of chromatographic separations was run at varying concentrations of formic, acetic, propionic and butyric acids in order to determine the approximate limits of detection and the linear dynamic range (see Table II). An unsulfonated resin column was used in conjunction with an aqueous eluent containing $4 \%$ butanol. The concentration of each analyte acid ranged from $2.5 \times 10^{-7} \mathrm{M}$ to $1.0 \times 10^{-2} \mathrm{M}$. Good separations with well-formed peaks were obtained for analyte concentrations of $1 \times 10^{-3}$ $\mathrm{M}$ and below. Significant peak fronting occurred at $5.0 \times 10^{-3} \mathrm{M}$ and $1.0 \times 10^{-3} \mathrm{M}$ concentrations, indicating some overloading. The peak shapes were very poor at 5.0 $\times 10^{-3} \mathrm{M}$ and $1.0 \times 10^{-2} \mathrm{M}$ concentrations with severe fronting. A chromatogram at an intermediate concentration $\left(1 \times 10^{-5} \mathrm{M}\right.$ of each analyte $)$ is shown in Figure 20.

Linear plots of peak area vs. concentration were obtained for each of the four analytes studied over most of the concentration range studied. Figure 21 shows a plot for formic acid with a linear regression correlation coefficient of 0.9995 between $1 \mathrm{x}$ $10^{-6} \mathrm{M}$ and $5 \times 10^{-4} \mathrm{M}$. A slight deviation from linearity is noted at $5 \times 10^{-7} \mathrm{M}$ and at $1 \times 10^{-3} \mathrm{M}$. Similar plots were obtained for acetic and propionic acids. The calibration plot for butyric acid has a non-linear region at the two lowest concentrations owing to interference from the overlapping carbonic acid peak (Figure 21). However, the butyric acid calibration plot is linear between $5 \times 10^{-6} \mathrm{M}$ and $5 \times 10^{-4} \mathrm{M}$. It should be possible to alter chromatographic conditions to minimize carbonic acid interference at very low butyric acid concentrations. 
Table II. Quantitative results showing linear dynamic range and linearity for formic, acetic, propionic, and butyric acids on an underivatized resin column $(150 \times 4.6 \mathrm{~mm})$ using a $4 \%$ butanol/water eluent. Detection is conductivity at $3 \mu \mathrm{S}$ full scale.

\begin{tabular}{|l|c|c|c|}
\hline \multicolumn{1}{|c|}{ Acid } & Linear Dynamic Range (M) & Corr. Coeff. & Slope \\
\hline Formic & $1.0 \times 10^{-6}-5.0 \times 10^{-4}$ & 0.9991 & 1.07 \\
Acetic & $1.0 \times 10^{-6}-5.0 \times 10^{-4}$ & 0.9998 & 0.92 \\
Propionic & $1.0 \times 10^{-6}-5.0 \times 10^{-4}$ & 0.9996 & 0.95 \\
Butyric & $5.0 \times 10^{-6}-5.0 \times 10^{-4}$ & 0.9993 & 0.89 \\
\hline
\end{tabular}


155

Figure 20. Chromatographic separation on underivatized PS-DVB resin column $(150 \times 4.6 \mathrm{~mm})$. Eluent conditions: $4 \%$ butanol/deionized water. Detection is conductivity with an output range of $3 \mu \mathrm{S}$ full scale. Flow rate is $1.0 \mathrm{ml} / \mathrm{min}$. Injected concentrations for all acids is $1 \times 10^{-5} \mathrm{M}$ and peaks are as identified. 


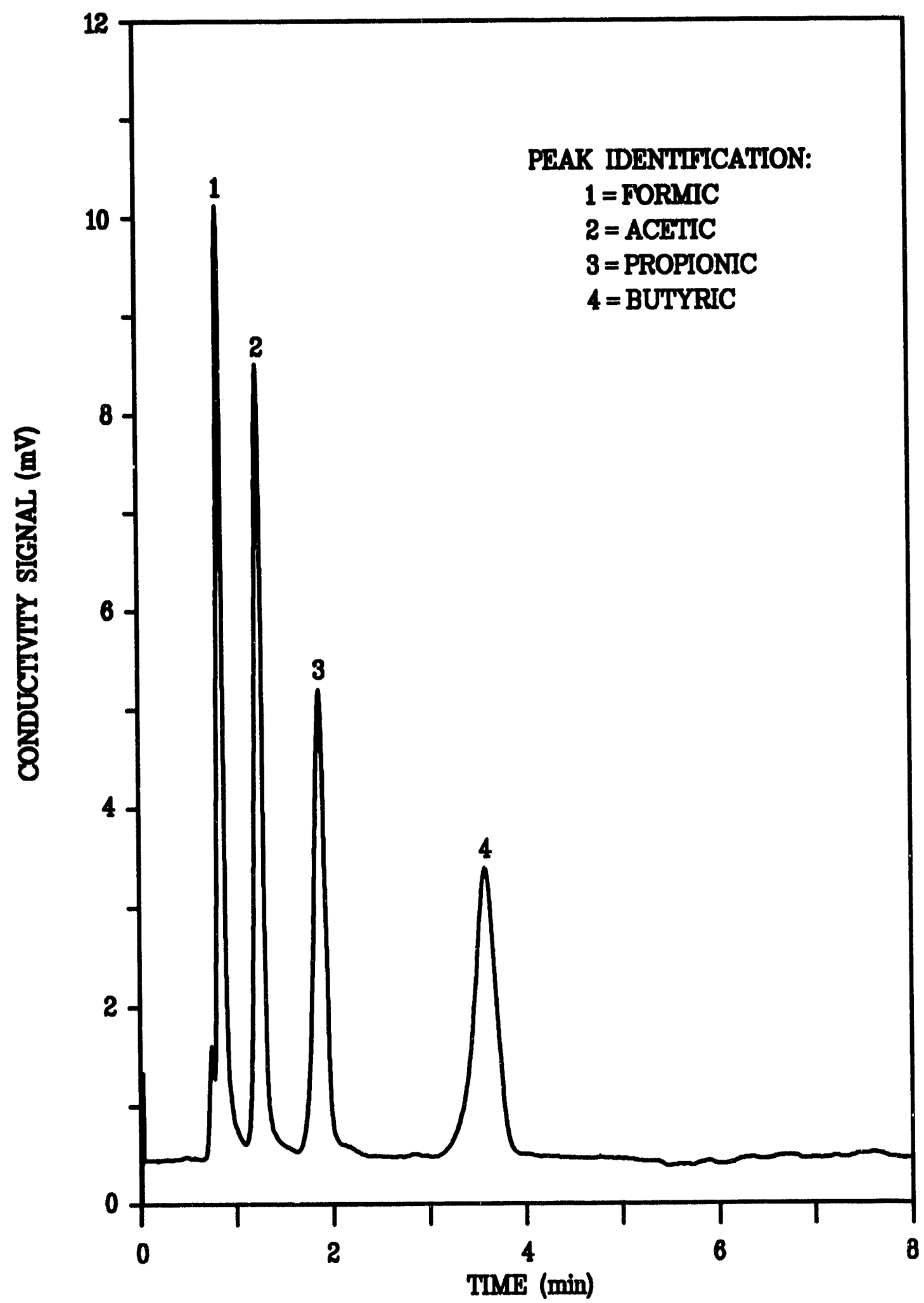


Figure 21. Plot of log concentration (M) versus log peak area for formic and butyric acids showing linear dynamic range. 


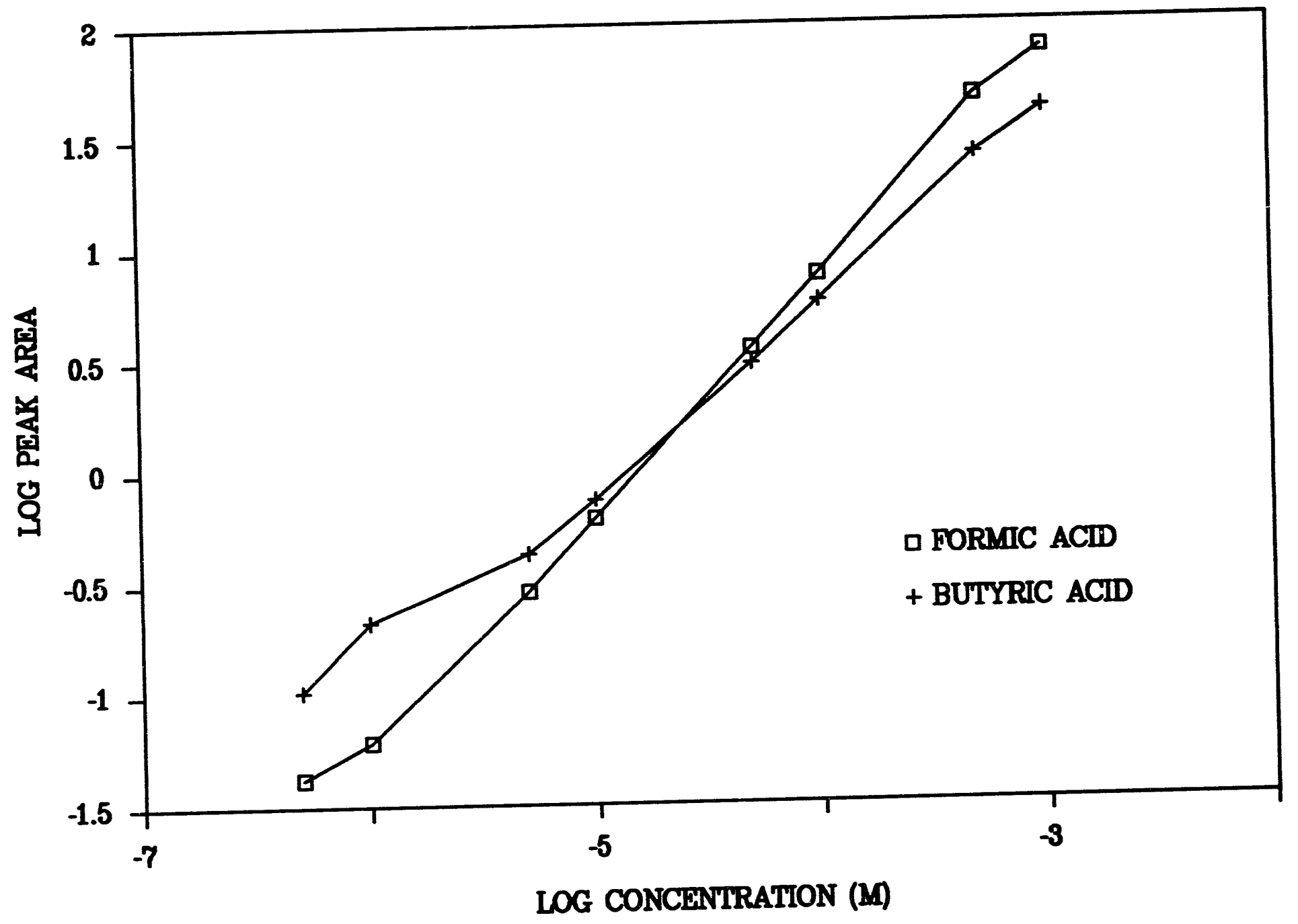


Limits of detection were estimated to be approximately $1 \times 10^{-7} \mathrm{M}$ for acetic and propionic acids and $5 \times 10^{-8} \mathrm{M}$ for formic acid, which has a somewhat sharper peak. This corresponds to approximately $2 \mathrm{ppb}$ for formic acid, $6 \mathrm{ppb}$ for acetic acid, and 7 ppb for propionic acid. 


\section{CONCLUSIONS}

The addition of surface modifiers to a predominantly aqueous eluent offers many exciting possibilities. The use of organic modifiers such as the straight-chain alcohols has shown some real promise in both a dynamic system and a semi-permanent system.

Carboxylic acids and other polar compounds are often separated by ionexclusion chromatography on gel-type ion-exchange resin columns. It has always been considered necessary to add a strong acid to the aqueous eluent to ensure that the carboxylic acid analytes are in the molecular form. We now conclude that separations of carboxylic acids can be performed on columns containing either underivatized or lightly sulfonated macroporous resins. The separation mechanism appears to be largely a hydrophobic attraction between the analytes and the resin. If a suitable alcohol or other organic moderator is added to the aqueous eluent, no strong acid is necessary. Excellent sensitivity and low detection limits are now possible for carboxylic acids using an ordinary conductivity detector.

Work done to this point shows that by varying the type and percentage of alcohol (or other modifiers) in the eluent, the retention time and peak shape of carboxylic acid species can be readily adjusted. The function of the organic moderator is to coat the resin surface and make it less hydrophobic. Butanol is more efficient than the lower straight-chain alcohols because it has a higher partition coefficient and lower concentrations in the eluent are thereby needed to effectively coat the resin. 
This concept may find extensive use for many other types of HPLC separations. In some cases the addition of surface modifiers may have clear advantages over chemically derivatized resins. It may be possible to alter the properties of a single resin by the appropriate choice of a chemical moderator in the eluent. This would make it possible to "fine tune" conditions to optimize the separation of interest.

Carbonic acid is another example of a chemical moderator that may be present in the eluent. Gas sparging does not remove low concentrations of $\mathrm{CO}_{2}$ from liquid eluents. Our work indicates that carbonic acid $\left(\right.$ or $\left.\mathrm{CO}_{2}\right)$ adsorbed on the resin surface can change the retention times of carboxylic acid analytes.

For systems with a semi-permanent coating, the modifier coating could be replenished periodically if needed or removed with a flush of pure organic solvent such as methanol. Initial work suggested that a reasonably stable coating of the resin surface can be achieved for use with purely aqueous eluent systems or with a small percentage of an organic solvent. The use of eluent modifiers to change the chemical properties of the stationary phase would be less costly and labor intensive than using different chemically modified stationary phases. 


\section{REFERENCES}

1. D. T. Gjerde and H. Mehra, Advances in Ion Chromatography, Vol. 1, p. 139, Century International, 1989.

2. P. R. Haddad and P. E. Jackson, Ion Chromatography, p. 195. Elsevier, Amsterdam, 1990.

3. J. S. Fritz, J. Chromatogr., 1991, 546, 111.

4. K. Tanaka, T. Ishizuka and J. Sunahara, J. Chromatogr., 1979, 174, 153.

5. B. K. Glód and W. Kemula, J. Chromatogr., 1986, 366, 39.

6. J.B. Morris and J.S. Fritz, $L C \cdot G C, 1993, \mathrm{xx}(\mathrm{x})$, xxx.

7. D.P. Lee and A.D. Lord, $L C \cdot G C, 1987,4,261$.

8. K. Tanaka, Personal Communications, March and October 1992.

9. E.D. Katz, C.H. Lochmüller, and R.P.W. Scott, Anal. Chem., 1989, 61, 349.

10. J.H. Knox and A. Pryde, J. Chromatogr., 1975, 112, 171.

11. R.M. McCormick and B.L. Karger, J. Chromatogr., 1980, 199, 259.

12. R.M. McCormick and B.L. Karger, Anal. Chem., 1980, 52, 2249.

13. E.H. Slaats, M. Markovski, J. Fekete, and H. Poppe, J. Chromatogr., 1981, 207, 299.

14. R.P.W. Scott and C.F. Simpson, Faraday Symp. Chem. Soc., 1980, 15, 69.

15. Y.M. Lau and C.F. Simpson, Anal. Proc. (London), 1988, 25(3), 86. 


\section{GENERAL CONCLUSIONS}

Macroporous polystyrene-divinylbenzene resins were chemically modified by simple organic reactions. These resins displayed improved hydrophilic properties making them particularly useful for HPLC applications. Chromatographic separations of metal cations were successful on a carboxylated resin using eluents containing an ethylenediammonium cation and a weakly complexing organic acid. The eluent $\mathrm{pH}$ was found to have a large effect on retention times of cationic species. This provided an additional parameter for optimization.

Separation of hydrophilic carboxylic acids and other small polar compounds was also possible using an appropriately functionalized macroporous polymeric resin column. An underivatized polymeric resin can be used, but greater retention and better separations are obtained using a resin with polar substituents, such as sulfonate or carboxylate. The separation mechanism seemed to be a partitioning of solutes between the mobile phase and the resin. Good separations were obtained on a carboxylic acid resin column under conditions where the resin carboxylic acid group would be mostly in the molecular rather than the ionized form. Separations were significantly faster on the macroporous resin columns than on the gel resin columns commonly used. In addition, solvent gradients were performed to give faster separations and sharper peaks for later-eluting compounds.

The addition of surface modifiers to a predominantly aqueous eluent offers many exciting possibilities. The use of organic modifiers such as the straight-chain 
alcohols has shown some real promise in both a dynamic system and a semi-permanent system. For the separation of the carboxylic acids demonstrated, there was a major improvement in detection sensitivity made possible by the use of conductivity detection. This may find extensive use for many other types of HPLC separations. In some cases, the addition of surface modifiers in the eluent may have clear advantages over chemically modified resins. This would make it possible to "fine-tune" the percentage of modifier to optimize the separation of interest. Work done to this point shows that by varying the type and percentage of alcohol (or other modifiers) in the eluent, the retention time and peak shape of carboxylic acid species can be readily adjusted. For systems with a semi-permanent coating, the modifier coating could be replenished periodically if needed or removed with a flush of pure organic solvent such as methanol. Initial work suggested that a reasonably stable coating of the resin surface can be achieved for use with purely aqueous eluent systems or with a small percentage of an organic solvent. The use of eluent modifiers to change the chemical properties of the stationary phase would be less costly and labor intensive than using different chemically modified stationary phases. 


\section{LITERATURE CITED}

1. P.R. Brown, Anal. Chem., 1990, 62, 995A.

2. C.E. Evans and V.L. McGuffin, J. Liq. Chromatogr., 1988, 11 (9 \& 10), 1907.

3. W.G. Kuhr and E.S. Yeung, Anal. Chem., 1988, 60, 2642.

4. M.J. Kessler, Am. Lab., June 1988, 86.

5. G. Horval and E. Pungor, Chromatography, 1987, 2(2), 15.

6. S. Hughes, P.L. Meschi and D.C. Johnson, Anal. Chim. Acta, 1981, 132, 1.

7. S. Hughes and D.C. Johnson, Anal. Chim. Acta, 1981, 132, 11.

8. A.L. Burlingame, D.S. Milington, D.L. Norwood, and D.H. Russell, Anal. Chem., $1990,62,268 R$.

9. K. Jinno and C. Fujimoto, J. Chromatogr., 1990, 506, 443.

10. M.M. Bushey and J.W. Jorgenson, Anal. Chem., 1990, 62, 978.

11. K. Grob, TrAC, Trends Anal. Chem., 1989, 8(5), 162.

12. A. Jaulmes and C. Vidal-Madjar, Advances in Chromatography, J.C. Giddings, E. Grushka, and P.R. Brown, Eds., Marcel Dekker, New York, 1989, Vol. 28, Chapter 1.

13. P.W. Carr, A.F. Bergold, D.A. Hangge and A.J. Muller, Chromatography Fonum, Sept.-Oct. 1986, 31.

14. T.M. Phillips, $L C \cdot G C, 1985,3,962$.

15. I.W. Warner, Chromatography Forum, Nov.-Dec. 1986, 55.

16. L.A. Haff, Chromatography, March 1987, 25.

17. A. Nahum and C.J. Horvath, J. Chromatogr., 1981, 203, 53.

18. J.B. Crowther and R.A. Hartwick, Chromatographia, 1983, $16,349$. 
19. K.K. Unger and U. Trüdinger, High Performance Liquid Chromatography, P.R. Brown and R.A. Hartwick, Eds., Wiley Interscience, New York, 1989, Chapter 3.

20. J.H. Knox and B. Kauer, High Performance Liquid Chromatography, P.R. Brown and R.A. Hartwick, Eds., Wiley Interscience, New York, 1989, Chapter 4.

21. P.W. Sutton and J.D. Kemp, Biochem., 1976, 15, 3153.

22. J. Porath, B. Olin, and B. Grandstand, Arch. Biochem. Biophys., 1983, 225, 543.

23. L. Sun and P.W. Carr, Abstract of Papers, Minnesota Chromatography Forum, 1993, Abstract 4.

24. D.J. Pietrzyk, High Performance Liquid Chromatography, P.R. Brown and R.A. Hartwick, Eds., Wiley Interscience, New York, 1989, Chapter 5.

25. R. Greyson and A. Patch, J. Chromatogr., 1982, 242, 349.

26. J.L. Glajch, J.J. Kirkland, and J. Köhler, J. Chromatogr., 1987, 384, 81.

27. M.A. Stadalius, J.S. Berus, and L.R. Snyder, $L C \cdot G C$, 1988, 6(6), 494.

28. J.J. Kirkland, C.H. Dilks, Jr., and J.E. Henderson, $L C \cdot G C$, 1993, 11(4), 290.

29. O. Mikes, J. Chromatogr., 1988, 41A, A142.

30. W. Heitz, J. Chromatogr., 1970, 53, 37.

31. F. Nevejans and M. Verzele, J. Chromatogr., 1987, 406, 325.

32. H. Small, T.S. Stevens, and W.C. Bauman, Anal. Chem., 1975, 47, 1801.

33. D.T. Gjerde, J.S. Fritz, and G. Schmuckler, J. Chromatogr., 1979, 186, 509.

34. D.T. Gjerde, J.S. Fritz, and G. Schmuckler, J. Chromatogr., 1980, 186, 509.

35. J.S. Fritz, D.T. Gjerde, and R.M. Becker, Anal. Chem., 1980, 52, 1519.

36. R.E. Barron and J.S. Fritz, Reactive Polymers, 1983, 1, 215.

37. D.L. Strong and P.K. Dasgupta, Anal. Chem., 1989, 61, 939.

38. D.L. Strong, C-U. Joung, and P.K. Dasgupta, J. Chromatogr., 1991, 546, 159. 
39. W.R. Jones, P. Jandik, and A.L. Heckenberg, Anal. Chem., 1988, 60, 1977.

40. J.S. Fritz, Anal. Chem., 1987, 59, 335A.

41. P.K. Dasgupta, Anal. Chem., 1992, 64, 775A.

42. R.C. Foley and P.R. Haddad, J. Chromatogr., 1986, 366, 13.

43. P.R. Haddad and R.C. Foley, Anal. Chem., 1989, 61, 1435.

44. G.J. Sevenich and J.S. Fritz, Anal. Chem., 1983, 55, 12.

45. C.H. Knight, R.M. Cassidy, B.M. Recoskie, and L.W. Green, Anal. Chem., 1984, $56,474$.

46. J.P. Sickafoose, Ph.D. Dissertation, Iowa State University, 1971.

47. R.M. Cassidy, Chem. Geol., 1988, 67, 185.

48. D. Yan, J. Zhang, and G. Schwedt, Fresenius Z. Anal. Chem., 1989, 335, 687.

49. A. Siriraks, H.M. Kingston, and J.M. Riviello, Anal. Chem., 1990, 62, 1185.

50. A.M. Dolognosov, T.V. Grishinova, and A.R. Timerbaev, Abstracts of Papers, International Ion Chromatography Symposium, Denver, CO, 1991, Abstract 16.

51. O.A. Shpigun and Y.A. Zolotov, Ion Chromatography in Water Analysis, Ellis Horwood Limited, Chichester, 1988.

52. P.R. Haddad and P.E. Jackson, Ion Chromatography:Principles and Applications, Elsevier, Amsterdam, 1990.

53. M. Miyazaki, K. Hayakawa, and S.G. Choi, J. Chromatogr., 1985, 323, 443.

54. H. Shintani, J. Chromatogr., 1985, 341, 53.

55. J.H. Sherman and N.D. Danielson, Anal. Chem., 1987, 59, 490.

56. M.E. Potts and J.R. Stillian, J. Chromatogr. Sci., 1988, 26, 315.

57. H. Sato, J. Chromatogr., 1989, 469, 339.

58. P. Kolla, J. Kohler, and G. Schomburg, Chromatographia, 1987, 23, 465. 
59. B. Kondratjonok and G. Schwedt, Fresenius Z. Anal. Chem., 1988, 332, 333.

60. P.E. Jackson, T. Bowser, and P.G. Alden, $L C \cdot G C$, 1992, 10(10), 786.

61. R.M. Wheaton and W.C. Bauman, Ind. Eng. Chem., 1953, 45, 228.

62. K. Tanaka, T. Ishizuka, and H. Sunahara, J. Chromatogr., 1979, 174(1), 153.

63. T. Jupille, M. Gray, B. Black, and M. Gould, Amer. Lab., 1981, 13(8), 80.

64. B.K. Glód and W. Kemula, J. Chromatogr., 1986, 366, 39.

65. G. Rumsby, J. Belloque, R.S. Ersser, and J.W.T. Seakins, Clin. Chim. Acta, 1987, 163(2), 171.

66. K. Tanaka and J.S. Fritz, J. Chromatogr., 1987, 409, 271.

67. D.T. Gjerde and H. Mehra, in Advances in Ion Chromatography, P. Jandik and R.M. Cassidy, Eds., Century International, Medfield, MA, 1989, Vol. 1, 139.

68. G. Albarran and C.H. Collins, J. High R hromatogr., 1989, 12(7), 486.

69. E. Papp and P. Keresztes, J. Chromatogr., 1ル, 506, 157.

70. J.S. Fritz, J. Chromatogr., 1991, 546(1-2), 111.

71. G.L. Zhao and L.N. Liu, Chromatographia, 1991, 32(9-10), 453.

72. J. Heard and K. Talmadge, Amer. Lab., 1992, May(1992), 24.

73. S. Mou, J. Jiang, and X. Hou, Sepu, 1992, 10(3), 133. (Chinese)

74. J. Yan and Y. Shi, Sepu, 1993, 11(1), 13. (Chinese)

75. V.T. Turkelson and M. Richards, Anal. Chem., 1978, 50, 1420.

76. B.K. Glód, P.R. Haddad, and P.W. Alexander, J. Chromatogr., 1992, 589(1-2), 209.

77. C. Pohlandt, S. Afr. J. Chem., 1982, 35(3), 96.

78. K. Tanaka and J.S. Fritz, Anal. Chem., 1987, 59, 708. 
79. T. Murayama, T. Kubota, Y. Hanaoka, S. Rokushika, K. Kihara, and H. Hatano, J. Chromatogr., 1988, 435(3), 417.

80. J. Haginaka, J. Wakai, and H. Yasuda, J. Chromatogr., 1988, 447(2), 373.

81. T. Okada, Anal. Chem., 1988, 60(17), 1666.

82. T. Okada and P.K. Dasgupta, Anal. Chem., 1989, 61(6), 548.

83. K. Tanaka and J.S. Fritz, J. Chromatogr., 1986, 361, 151.

84. T. Okada, Anal. Chim. Acta, 1990, $230(1), 9$.

85. H.F. Botte and L. Joergensen, J. Chromatogr., 1992, 602(1-2), 27.

86. R. Widiastuti, P.R. Haddad, and P.E. Jackson, J. Chromatogr., 1992, 602(1-2), 43.

87. B.K. Glód, P.R. Haddad, and P.W. Alexander, J. Chromatogr., 1992, 595(1-2), 149.

88. H. Itoh and Y. Shinbori, Bull. Chem. Soc. Jpn., 1985, 58(11), 3244.

89. K. Hayakawa, K. Nomura, and M. Miyazaki, Anal. Sci., 1992, 8(1), 11 i.

90. P.R. Monk and P.G. Iland, Food Technol. Aust., 1984, 36(1), 18.

91. P.R. Monk and P.G. Iland, Food Technol. Aust., 1984, 36(1), 16.

92. K. Fujimura and M. Tsuchiya, Bunseki Kagaku, 1988, 37(10), 549. (Japanese)

93. C.A. Accorsi and G. Blo, J. Chromatogr., 1991, 555(1-2), 65.

94. S. Tanaka, K. Yasue, and Y. Hashimoto, Bunseki Kagaku, 1990, 39(1), 13. (Japanese)

95. T.R. Fox and N.B. Comerford, Soil Sci. Soc. Am. J., 1990, July-August(1990), Vol. 54, 1139.

96. T. Morikami, S. Tanaka, Y. Hashimoto, T. Inomata, and Y. Hanaoka, Anal. Sci., 1991, 7, 1033.

97. D. Grosjean, A. Van Neste, and S.S. Parmar, J. Liq. Chromatogr., 1989, 12(15), 3007. 
98. W.A. Byers, S.L. Anderson, and W.M. Hickam, Proc.-Int. Water Conf., Eng. Soc. West. Pa. $44^{\text {th }}, 1983,436$.

99. A.D. Karpyuk, S.F. Betekhtin, and A.V. Yablochkin, Zavod. Lab., 1992, 58(4), 8. (Russian)

100. R. Gelius, M. Ahrens, C. Troeltzsch, Z. Chem., 1979, 19(7), 260. (German)

101. R. Alen, E. Sjostrom, and S. Suominen, J. Chem. Technol. Biotechnol., 1991, 51(2), 225.

102. G. Iwinski and D.R. Jenke, J. Chromatogr., 1987, 392, 397.

103. M. Fukui, H. Konishi, K. Ohta, and K. Tanaka, Bunseki Kagaku, 1992, 41(3), T27. (Japanese)

104. G.R. Noll, N.J. Nagle, D.J. Mitchell, J.O. Baker, K. Grohmann, and M.E. Himmel, J. Liq. Chromatogr., 1990, 13(4), 703.

105. L.A. Kaine, J.B. Crowe, and K.A. Wolnik, J. Chromatogr., 1992, 602(1-2), 141.

106. J. Lehotay and M. Traiter, J. Chromatogr., 1974, 91, 261.

107. D.P. Lee and A.D. Lord, $L C \cdot G C, 1987,4,261$.

108. P.Walser, J. Chromatogr., 1988, 439, 71.

109. L.R. Snyder, J. Chromatogr., 1974, 92, 223.

110. L.R. Snyder, J. Chromatogr. Sci., 1978, 16, 223.

111. P.J. Schoenmakers, H.A.H Billiet, and L. de Galan, J. Chromatogr., 1981, 218, 259.

112. S.P. Bakalyar, R. Mcllwrick, and E. Roggendorf, J. Chromatogr., 1977, 142, 353.

113. E. Roggendorf and R. Spatz, J. Chromatogr., 1981, 204, 263.

114. M. Waksmundzka-Hajnos, J. Chromatogr., 1992, 623(1), 15.

115. V.M. Staroverov, V.I. Deineka, and L.V. Krichkovskaya, Khim.-Farm. Zh., 1990, 24(9), 85. (Russian) 
116. M.H. Gaffney, R.M. Stiffin, and I.W. Wainer, Chromatographia, 1989, 27(1-2), 15.

117. I.M. Wainer, R.M. Stiffin, and T. Shibata, J. Chromatogr., 1987, 411, 139.

118. I.M. Wainer, M.C. Alembik, and E. Smith, J. Chromatogr., 1987, 388(1), 65.

119. P.V. Bhat, T.C. Ha, and A. Lacroix, J. Chromatogr., 1983, $260(1), 129$.

120. M. Enquist and J. Hermansson, J. Chromatogr., 1990, 519(2), 271.

121. A. Munoz de la Pena, T.T. Ndou, V.C. Anigbogu, and I.M. Wainer, Anal. Chem., 1991, 63(10), 1018.

122. R.M. Mohseni, R.J. Hurtubise, J. Chromatogr., 1990, 499, 395.

123. M.A. Tarr, G. Nelson, G. Patonay, and I.M. Warner, Anal. Lett., 1988, 21(5), 843.

124. M.G. Khaledi, Anal. Chem., 1988, 60(9), 876.

125. F.P. Tomasella, J. Fett, and L.J.C. Love, Anal. Chem., 1991, 63(5), 474.

126. J. Maslowska and G. Bazylak, Acta Univ. Lodz., Folia Chim., 1991, 9, 93.

127. J.J. Naleway and N.E. Hoffman, J. Liq. Chiomatogr., 1981, 4(8), 1323.

128. E. Heldin, N.H. Huynh, and C. Pettersson, J. Chromatogr., 1991, 585(1), 35.

129. W.J. Cheong and P.W. Carr, J. Liq. Chromatogr., 1987, 10(4), 561.

130. J.J. Michels and J.G. Dorsey, J. Chromatogr., 1988, 457, 85.

131. Y.M. Lau and C.F. Simpson, Anal. Proc. (London), 1988, 25(3), 86. 


\section{ACKNOWLEDGEMENTS}

I would first like to acknowledge Dr. James S. Fritz, my major professor. You have been a good friend and an excellent teacher. Your guidance and support has helped me to develop confidence as a scientist. You have a real love for the work that you do which inspired me to work that much harder. The fact that we could talk about things other than science on a daily basis also meant alot to me. I wish you well in all of your future endeavors.

I would also like to thank my committee members, Drs. Houk, Johnson, Kraus, and Oulman. I appreciate you taking time from your busy schedules to serve on my committee. Dr. Johnson, you made me feel comfortable to be at ISU when I first started and felt a little unsure of myself. Sam, I really enjoyed our friendship and fun times we had playing basketball and softball. I would definitely hold out for the big bucks in your free agency next year. Anybody who hits the ball and can make freethrows like you deserves to make more than Johnny Orr.

I would like to thank my soon-to-be wife, Lisa, for all of her love and support. My life has gotten better every day since you came into it. You have made many sacrifices for me and all of them are greatly appreciated. You are a very special person and I am excited about our future lives together. I hope Chicago has a good supply of jalapeno peppers!

I would like to thank my mother, Jeanette, for her tremendous display of courage and strength. I will always think of you as a role model as I face life's ups and 
downs. Your love was always present as you taught me good values as a child. You have a great way of making the best of any situation and that is really encouraging.

I have several friends in Ames that deserve a special thanks. Dave Mcgregor, Luther Schmidt, Rich Roberts, Nancy Benz, Phil Dumont, Mojo, Oden Warren, Doug Wigert, Nancy and Jerry Gilinsky, and the rest of the Fritz group members. Each of you has contributed in some way to making my stay in Ames not just bearable, but enjoyable.

Dave, I could write pages about the fun memories we've made together. This isn't necessary, however, since the stories seem to be told on a frequent basis. I laugh every time. You have been a great help with your vast knowledge of life. I guess that comes with experience (and age?). I'm really happy that you'll be in Chicago with us.

Luther, my officemate for 5 years and roommate for 2 years, you have been a good friend and colleague. I have never met anyone with such a knowledge of sports (although, Oden you are very close). Maybe the two of you should collaborate on a book. I thank you for introducing me to Rush and for always making life in the office interesting. I wish you and Andrea the best as you start your new lives in ArKANSAS.

Rich, you have a great sense of humor. You also have alot of courage for marrying a redhead (sorry Carla, I just had to say something). I really enjoyed rooming with you and taking part in the fun times we had together. Don't forget my share of royalties if you ever decide to market our special secret party tape. I wish you and Carla the best in your future together. 
Nancy, you have been a good friend and an invaluable source of help at work. You tolerate quite a bit, which is not bad for a cheese (no, I won't come right out and say it - pretty nice of me, EH?). If it weren't for your decision-making abilities, the lunch bunch would probably wander campustown for days with empty stomachs. I think you are a very thoughtful person and you will go far in life. Good luck with fantasy football and that stitching thing (no more material from me!)

Phil, it was alot of fun doing the intramural sports and golfing with you. I hope that you and Lisa enjoy the rest of your stay here and that things go well for you. If not, I think you could have a promising future as a referee.

Mojo, I'm happy to say that I know somebody called Mojo. It's been a blast! I hope you enjoy your remaining time in Ames and I wish you well in your pursuits. Come visit Dave and I in Chicago some time. I know we would both love to have you. Oden, it has been fun getting to know you in the last couple of years. Beth will probably be relieved to know that the "bad influerces" are leaving town. I wish you, Beth, and Fletcher the best in your future.

Doug, it was great to get to know you. I enjoyed the dunk-ball, golf, etc. but most of all I enjoyed railing on Jerry with you. You would call at just the right times to get me out of the lab for a break (kept me from becoming another Dean). Best wishes to you and your family.

Nancy and Jerry, you guys are really good friends. I'm glad that our paths crossed in Ames for awhile. It's nice that we will be closer to you guys again when we move to Chicago. Good luck with your upcoming bundle of joy! Jerry, now that I have 
finished this work, I will have some time to begin a second thesis (undoubtably a multivolume set) on you and all of your little shenanigans. Keep your eyes open - it should be a best seller.

I also want to acknowledge my close friends from the neighborhood, Dave Feuerstein, Konrad Gastony, and John Peterson. We've been good friends for a long time. We've had alot of fun over the years and I expect we'll have many good times in the years to come. Your friendships mean alot to me so I hope we always keep in touch. I wish the best success to all of you.

I couldn't leave without expressing my appreciation to Cyclone basketball and hockey. These two sports delivered many hours of entertainment. I'll watch to see if ISU will adopt a football program some day. Go Clones! Beat the Hawks!

I would like to thank Doug Gjerde of Sarasep, Inc. and Dan Lee of the Hamilton Co. for their gifts of resins and columns that were important for the completion of my research. I would also like to thank Ames Laboratory and Supelco, Inc. for their financial support.

This work was performed at Ames Laboratory under contract no. W-7405-eng-82 with the U. S. Department of Energy. The United States government has assigned the DOE Report number IS-T 1667 to this thesis. 

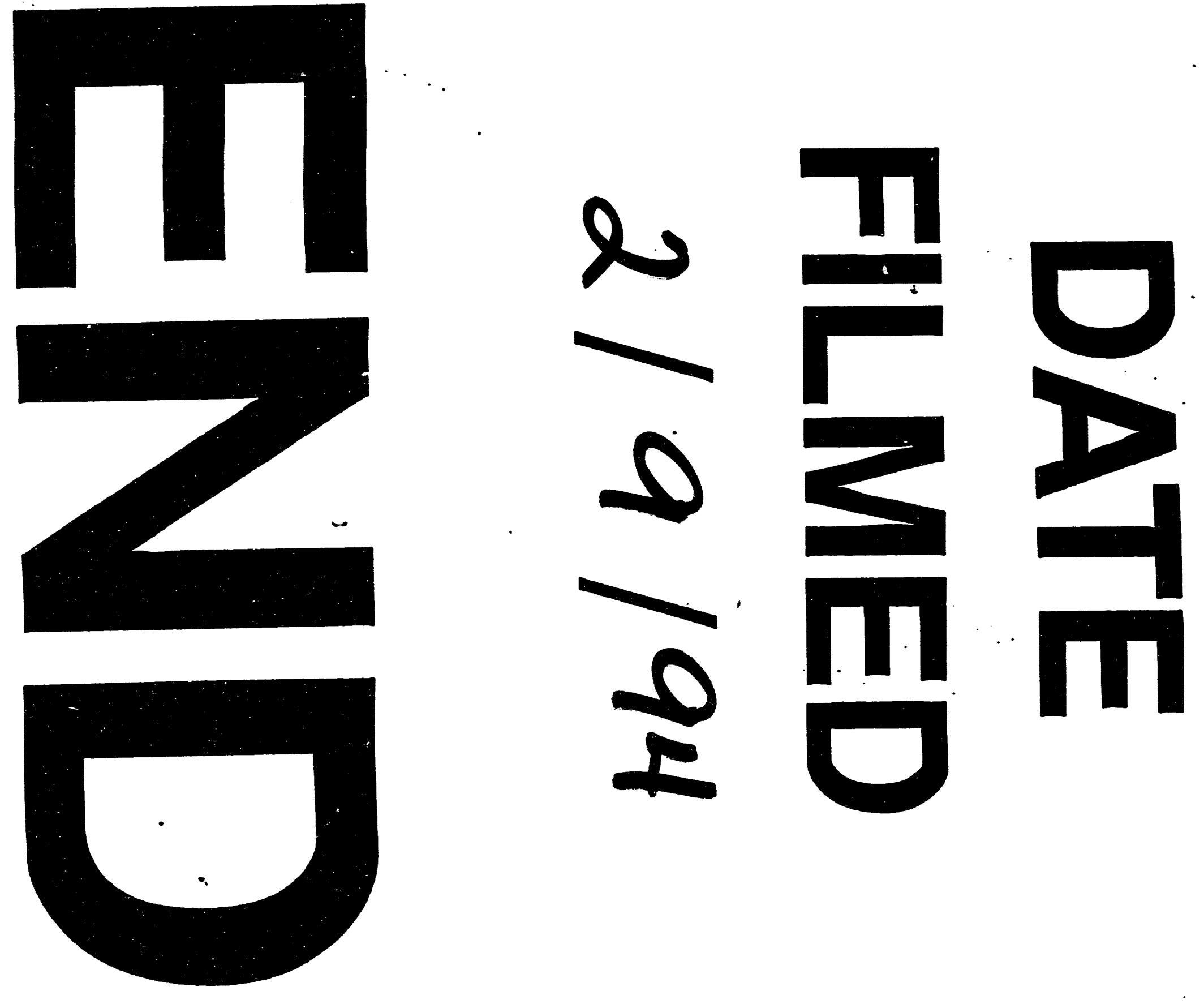


$$
\text { L }
$$

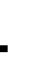

